
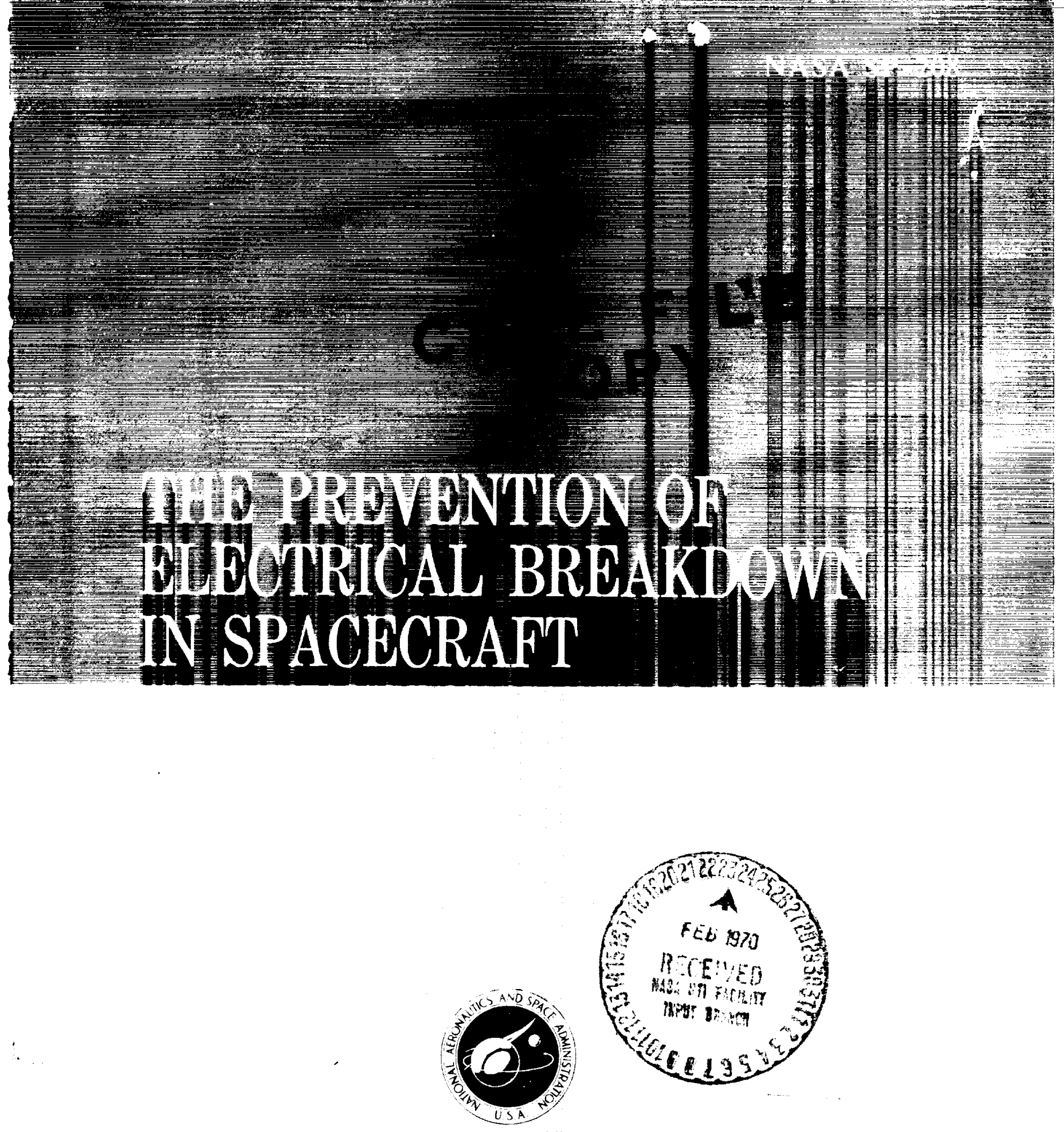

NATIONAL, AERONAUTICS AND SPACE AIMMINISTRATION 


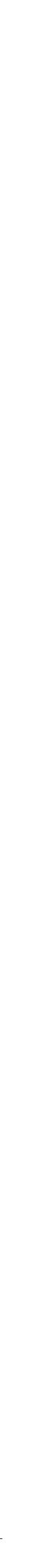


NASA SP-208

\section{THE PREVENTION OF ELECTRICAL BREAKDOWN IN SPACECRAFT}

by

Fred W. Paul and Donald Burrowbridge

Goddard Space Flight Center Greenbelt, Md. 
For sale by the Clearinghouse for Federal Scientific and Technical Information Springfield, Virginia 22151 - Price $\$ 3.00$ 


\begin{abstract}
This paper on high voltage electrical breakdown provides a basic understanding of the phenomena and problems encountered in the use, design, and fabrication of high potential systems for spacecraft. The material was compiled from the literature listed in the bibliography and from conferences with experienced personnel at Goddard Space Flight Center; Ames Research Center, Moffett Field, California; Jet Propulsion Laboratory, Pasadena, California; and the University of Chicago.
\end{abstract}




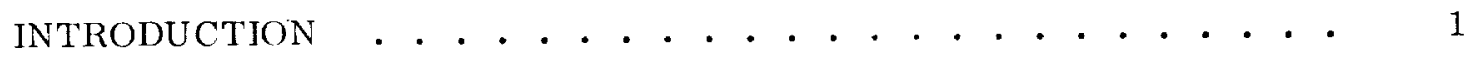

PHENOMENA ASSOCIATED WITH ELECTRICAL BREAKDOWN • • . 1

Prebreakdown Phenomena in Gases . . . . . . . . . . 1

Breakdown Phenomena in Gases . . . . . . . . . . . . 4

Emission of Electrons and Ions by Solids . . . . . . . . . 16

Electrical Breakdown in Liquids . . . . . . . . . . . . 18

Electrical Breakdown in Solids . . . . . . . . . . . 18

Summary of Electrical Breakdown Phenomena. . . . . . . . 28

DESIGN PRINCIPLES . . . . . . . . . . . . . . . . . 29

Approach ..................... . . 29

Designing to Avoid Breakdown . . . . . . . . . . . . 29

Designing to Minimize Damage .............. . 40

Designing for Frequent Testing . . . . . . . . . . . 41

DESIGN AND FABRICATION PRACTICES . . . . . . . . . . 42

Three Examples . . . . . . . . . . . . . . 42

High Frequency Prictices . . . . . . . . . . . 52

Conformal Coating Procedures . . . . . . . . . . . 54

Connectors ................... 58

Recommendations from JPL. . . . . . . . . . . 59

Approved Practices . . . . . . . . . . . . 60

TEST CONSIDERATIONS . . . . . . . . . . . . . 62

Test Opportunities . . . . . . . . . . . . . 62

Testing Problems ................. . 63

Simple Specification . . . . . . . . . . . . . 64

DESIGN REVIEW CHECKLIST. . . . . . . . . . . . . . 90

Checklist Questions. . . . . . . . . . . . . 90

ACKNOWLEDGMENTS . . . . . . . . . . . . . . . . 92 


\section{CONTENTS (Cont'd)}

$\underline{\text { Page }}$

References . . . . . . . . . . . . . . . . . 93

Bibliography . . . . . . . . . . . . . . . . . 95 


\section{LIST OF ILLUSTRATIONS}

$\underline{\text { Figure }}$

$\underline{\text { Page }}$

1 Applied voltage versus current for a typical gas discharge ... 5

2 Paschen's Curve of sparking voltage as a function of the product of gas pressure and electrode spacing . . . . . . . 6

$3 \quad$ Electric field between electrodes in the presence of a uniformly distributed space charge . . . . . . . . 7

4 A typical glow discharge . . . . . . . . . . . 10

$5 \quad$ High Frequency $(\sim 100 \mathrm{MHz})$ gas breakdown characteristics . . 15

6 The values of electric field and frequency for which multipacting may occur . . . . . . . . . . . . . 16

7 (a) Equivalent circuit of a dielectric containing a void.

(b) Time dependence of applied voltage, $V$. (c) Time dependence of void voltage, $\mathrm{v}_{\mathrm{V}}$. . . . . . . . . . . 26

$8 \quad$ Breakdown voltage gradient versus electrode separation in air at atmospheric pressure (Reference 9) . . . . . . . 30

$9 \quad$ Possible regions of multipacting between parallel plates (Reference 10) . . . . . . . . . . . . 31

10 Sharp edges and points must be avoided . . . . . . . 43

11 Cockroft-Walton generator and multiplier phototube . . . . 44

12 Spaced parallel wires avoid gas pockets in potting . . . . 46

13 Voids in potting compound cause trouble . . . . . . . . 47

14 Proper priming results in good adhesion between hardware and potting compound ............ . . 50

$15 \quad$ Illustration of direct contact 15 kilovolt assembly . . . . . 51

16 Paschen's Curve as modified by multipacting . . . . . 52

17 Vented electrical connectors avoid trapped gas . . . . . 58 


\section{LIST OF TABLES}

Table

Page

1 Generalizations of Breakdown in Gases, Liquids and Solids................. . . . 19

2 Dielectric Strength and Arc Resistance for Selected Insulation Materials Suitable for Molding, Extrusion or Casting . . 34

3 Gases Suitable for High Voltage Systems in Hermetically Sealed Containers . . . . . . . . . . . . 36 


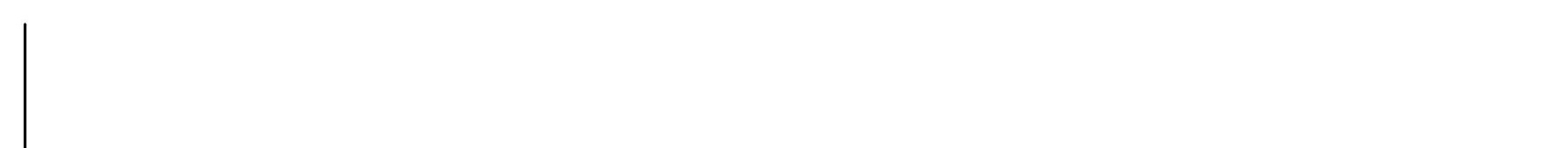




\title{
THE PREVENTION OF ELECTRICAL BREAKDOWN IN SPACECRAFT
}

\author{
by \\ Fred W. Paul and Donald Burrowbridge \\ Goddard Space Flight Center
}

\section{INTRODUCTION}

Many unusual problems arise when high potentials are used at reduced pressures, and not all of the basic mechanisms are well understood. The first part of this report is intended to give a general knowledge of fundamental breakdown phenomena, with particular emphasis on gas discharges. Other parts contain some design techniques, principles, and practices that have been employed successfully in space applications. The art of building high voltage systems has developed to the point where guidelines can be given to help the new designer avoid old problems. The problems and techniques of testing are also discussed, and a rudimentary checklist is included as a suggested aid in the ready review of specific design considerations and problem areas. A sample design specification from Jet Propulsion Laboratory is included.

\section{PHENOMENA ASSOCIATED WITH ELECTRICAL BREAKDOWN}

Electrical breakdown can occur in the gaseous, liquid, and solid states of materials. It is best understood and described as it relates to gases. The object here is to give an intuitive understanding of electrical breakdown, especially in gas.

\section{Prebreakdown Phenomena in Gases}

\section{Gases as Insulators}

In the gaseous state, atoms and molecules form an almost perfect insulator. The dielectric constant is very near unity and the energies of collision are of the order of 0.03 electron volts (ev) at $0^{\circ} \mathrm{C}$. Gas cannot become a conductor unless some mechanism can ionize it, since ions and/or electrons are required to carry a current in a gas. The energy required for ionization ranges from $3.5 \mathrm{ev}$ for the most easily ionized gases to $24.5 \mathrm{ev}$ for helium. 
Environmental factors produce some ionization of the air, amounting to perhaps 20 ions per cubic centimeter per second from all causes. Due to the fact that some ions are lost and more are continually being formed there is an equilibrium value of about 1000 ions of each sign per cubic centimeter of air at sea level.

When a gas is placed between conducting plates and a voltage is applied, a current will flow as long as the gas is ionized. This current is composed of positive ion currents and electron currents, which are attracted to the negative and positive plates respectively.

Free electrons in gases in the presence of an electric field exhibit both random and directed motions. The random motions caused by thermal oscillations cause on the order of $10^{11}$ collisions per second with molecules at S.T.P. Superposed on this random motion is a directed drift velocity produced by the imposed electric field. The electrons gain energy from the electrical field and lose energy in collisions with molecules. The number of impacts per second is a complicated function of the energy of the electron, the molecular species, and the gas density. Consequently, the energy of electrons in an electric field varies with field strength, $E$, and pressure, p. The electron energies are distributed about a mean value which is a function of $\mathrm{E} / \mathrm{p}$, and of absolute temperature, $T$. The distribution of energy among the electrons is not of the conventional Maxwellian form, and the functional dependence on $\mathrm{E} / \mathrm{p}$ in many instances even varies with $\mathrm{E} / \mathrm{p}$. In some simple cases the distribution of energies can be computed.

The electron drift velocity is much greater than that for ions. At atmospheric pressure, the electron drift velocity in nitrogen is of the order of $10^{4} \mathrm{~cm} / \mathrm{sec}$ per volt $/ \mathrm{cm}$, compared to $2.2 \mathrm{~cm} / \mathrm{sec}$ per volt $/ \mathrm{cm}$ for molecular ions.

\section{Townsend's First Coefficient}

As voltage is increased with a given gas ionization between two charged plates, the current approaches what appears to be saturation. However, at even higher values of the electric field, the current again continues to increase. The region where this second increase occurs is dependent on pressure and on the gas being used. For air, the second current increase begins at $\mathrm{E} / \mathrm{p}=20$; in hydrogen, at $\mathrm{E} / \mathrm{p}=10$; and in pure argon and neon, at $\mathrm{E} / \mathrm{p}=5$ and 2 respectively. The increase in current with increasing voltage is exponential and is terminated by a spark or other sudden electrical breakdown. 
This phenomenon was explained by J.S.E. Townsend as follows. Some of the electrons gain enough energy from the electric field, despite collision losses, to ionize the gas molecules. Each ionization releases another electron. Both the new electron and original continue to produce further ionization. Thus the number of free electrons will double for ionization distance covered provided no electrons are lost by recombination or other mechanisms. This process is called cumulative ionization. It leads to the equation for the current

$$
\mathrm{i}=\mathbf{i}_{\mathrm{o}} \mathrm{e}^{\alpha \mathrm{x}}
$$

where $i_{o}$ is the initial electron current at the cathode, $\alpha$ is the number of new electrons released by ionization per centimeter by one electron, and $\mathrm{x}$ is the distance from the cathode in centimeters. $\alpha$ is Townsend's first coefficient.

\section{Townsend's Second Coefficient}

Equation 1 describes the current increase to apparent saturation. The second rapid increase occurs only at large values of $\mathrm{E} / \mathrm{p}$. Townsend explained the second increase of current in terms of a secondary process. Several other secondary processes do occur. Two of the most important are: The release of secondary electrons from the cathode by the impact of positive ions; and photoelectric action. (The photons come from the gas excited by electron impacts.) The equation for positive-ion bombardment as a process for electron release is

$$
i=i_{0} \frac{e^{\alpha x}}{1-\gamma e^{\alpha x}},
$$

where $\gamma$ is the probability that a positive ion will release an electron. This is Townsend's second coefficient. The other quantities in the equation are the same as those in Equation 1. There is a similar equation for photoelectric effects. In general, $\gamma$ increases with $\mathrm{E} / \mathrm{p}$, but becomes appreciable only at much higher values of $\mathrm{E} / \mathrm{p}$ than does $\alpha$.

\section{Gaseous Ions}

There are several kinds of gaseous ions. In the ionization processes discussed above, an electron is separated from a molecule leaving the molecule as a positively charged ion. Certain kinds of gas molecules, for example, $\mathrm{O}_{2}$ and $\mathrm{SO}$, can capture a free electron and become negatively charged ions. In the presence of some impurities in the gas, ions formed by combination of two or more molecules or molecular fragments can exist. In an electric 
field, gaseous ions accelerate more slowly than electrons because of their larger mass and greater collision cross-section. In the absence of an electric field, gaseous ions disappear by either diffusion to the walls of the chamber where their charge is neutralized, or by recombination of positive and negative ions. When there is an electric field, the gaseous ions are accelerated toward one or the other of the electrodes and under certain circumstances the flow of gaseous ions makes a significant contribution to the electric current.

\section{Space Charge}

When an electric field is imposed on a region where there are electrons and positive ions, the electrons tend to be quickly carried away toward the positive electrode while the positive ions, which accelerate much more slowly, are left behind. The resulting accumulation of ions is called a space charge. When both positive and negative gaseous ions are present we may have a space charge of either sign. In the presence of a space charge the electric field between the electrodes results from both the applied voltage and the space charge. Space charge tends to decrease current for a given applied voltage.

\section{Breakdown Phenomena in Gases}

A general description of electrical discharge phenomena in gases is most conveniently presented by reference to Figure 1 , which shows the relation between applied voltage and measured current over a wide range of current values. At small values of current from $10^{-10}$ amps to $10^{-7}$ amps the current is independent of the applied voltage and depends only on the rate of production of ions in the gas. At low current values the gas exhibits little or no luminosity because the number of radiating atoms or molecules is small. This current range is called the Townsend discharge. As the current is increased from $10^{-7}$ to $10^{-5}$ amperes the voltage drops and more luminosity appears. Note that the voltagecurrent relationship is just the opposite of ohmic: as the current increases the voltage decreases. As current increases to $10^{-3}$ amperes, or even greater, there is another region of independence of current and voltage. This is called the normal glow discharge region. With still greater current the voltage rises again to higher values than initially and the luminous glow becomes brighter. When the current reaches a peak at about $10^{-2}$ amperes, the voltage begins to fall rapidly to a low constant value as current goes to several tens of amperes; this is the arc discharge region. The detailed values of current and voltage at which these various phases of the discharge occur depend on the gas used, the external resistance, the geometry and nature of the electrodes, and several other factors. 
Figure 1 - Applied voltage versus current for a typical gas discharge.

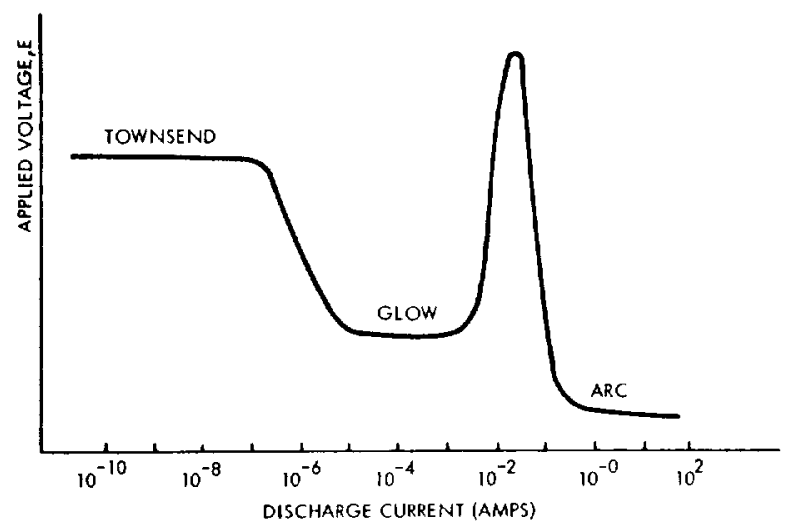

\section{Paschen's Law}

In 1889, F. Paschen made an experimental discovery that helps generalize the complex subject of gas breakdown. Paschen's Law simply states that in a uniform field the sparking potential of a gas depends only on the product of the gas pressure, $P$, and the electrode separation, $\delta$. Mathematically this is $V_{\mathrm{S}}=\Psi(\mathrm{p} \delta)$ where $\mathrm{V}_{\mathrm{S}}$ is the sparking potential and $\Psi$ is a function of $\mathrm{p} \delta$ only. An alternative way of stating Paschen's Law is $V_{S}=\Psi(n \delta)$ where $n$ is the number of molecules per cubic centimeter; this takes into account temperature changes. It should be remembered that Paschen's Law applies only to breakdown in uniform electric fields (see Figure 2).*

\section{Breakdown in Nonuniform Fields}

In nonuniform electric fields, i.e., fields which vary from place to place in space, electrical breakdowns occur more readily than for the uniform fields to which Paschen's Law applies. Nonuniform fields exist if the electrodes are not parallel, or if one is a point and the other a plane, or for a wire and a coaxial cylinder. Since nonuniform fields are much more frequently encountered in practical circuits, it is important to consider how these nonuniformities affect electrical breakdown phenomena.

When the size of the anode is small compared to the length of the electron mean free path, the electrons may not strike it following their last molecular collision, and may cause some additional ionization before being collected.

*For exceptions to Paschen's Law c.f. Von Hipple, p. 187, The Molecular Designing of Materials and Devices, MIT Press, Cambridge, 1965. 


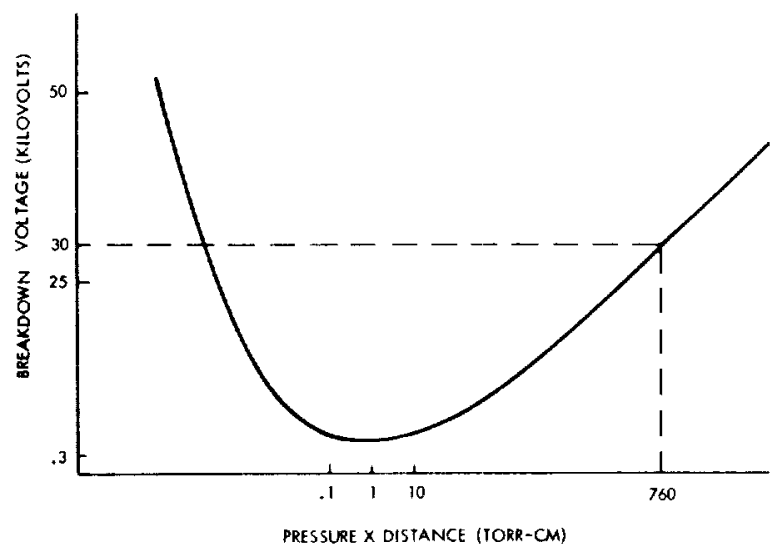

Figure 2 - Paschen's Curve of sparking voltage as a function of the product of gas pressure and electrode spacing.

For dissimilarly shaped electrodes (wire and coaxial cylinder, for example), the value of $\gamma$ (the second Townsend coefficient) will depend on the polarity of electrodes. If the small electrode is the cathode, then $\gamma$ is generally large and a lower value of the breakdown voltage results.

The Law of Similitude, or the Similarity Principle, states that the voltampere characteristic of a given gas discharge system will not change if all the linear dimensions of the system are increased by a constant factor and the gas pressure is reduced by the same factor, or vice versa. This principle may be used to considerable advantage in the analysis of many discharge phenomena; for example, by dimensional analysis. If the Law of Similitude is applied to the case of breakdown along a uniform field path, it becomes a statement of Paschen's Law. For the Law of Similitude to be obeyed, all processes which determine discharge behavior must be functions of $\mathrm{E} / \mathrm{p}$ only.

When space charge is present, it is possible to have nonuniform fields even though a uniform field geometry is used. The generation of nonuniform fields in this manner requires unequal concentrations of positive and negative charge. This is not uncommon in gas discharges.

It is useful to consider a uniform charge density between electrodes. The effect of uniform charge density is shown in Figure 3 where field is increased at the cathode and reduced by a like amount at the anode.

An ion current density of $3 \times 10^{-5} \mathrm{amp} / \mathrm{cm}^{2}$ can typically cause a 10 percent cathode field increase.

In a Townsend discharge, collisional ionization does not lead to a uniformly distributed positive space charge. Most of the space charge buildup occurs in the vicinity of the cathode. 
Figure 3 - Electric field between

electrodes in the presence of a

uniformly distributed space charge.

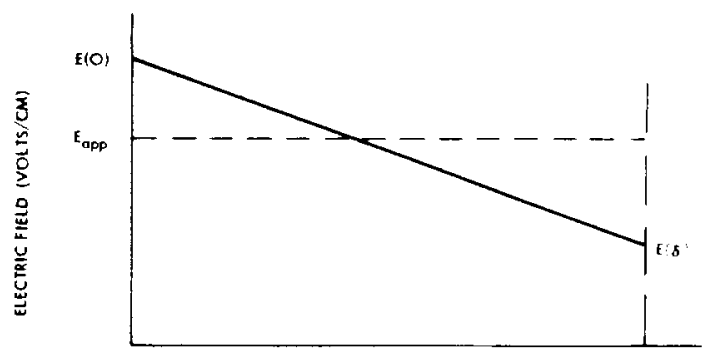

DISTANCE FROM CATHODE $\mathrm{KCM}$

Time Lags for Breakdown

Up to this point we have been dealing with steady state situations. It has been assumed that the time of application of voltage plays no part in determining the prebreakdown currents or the breakdown threshold. This may not always be the case. When a field sufficiently large to cause breakdown is applied to a system of electrodes, there are two reasons why sparkover does not occur immediately: First, time is required for one or more initial electrons to appear in a favorable gap position to initiate the necessary avalanches; and second, the development of the avalanches and buildup of current takes some time because of the finite particle mobilities. Thus there is a time delay before breakdown that is the sum of these two periods. The first is called the statistical time lag and the second the formative time lag.

There is always a natural background of ionizing radiation due to cosmic rays and radioactive impurities.

For a gap which depends upon this natural environment as the sole source of electrons the time lag will be largely statistical. In a gap purposely irradiated, the statistical time lag may be sufficiently reduced so that the total lag is composed primarily of the formative part. The two time lags are separated and measured in just this manner. These time lags are of considerable practical as well as theoretical interest, since, if the duration of the applied voltage pulse approaches the time lag, an appreciable increase in breakdown voltage may be observed (Reference 1). Chapter 8 of reference 1 is devoted to the time lag topic and will provide a detailed treatment for the interested reader.

\section{Direct Current Discharge Types}

The various types of direct current discharge in gases will now be described in greater detail. A transition from a quiet, nonluminous Townsend discharge to a more energetic discharge will occur when the current grows by the release 
of more charged particles as governed by Equation 1; it will increase even more rapidly if the secondary emission from the cathode becomes an important factor so that the relation between initial current and final current must be described by Equation 2. Townsend's criterion for a voltage breakdown is that the denominator of Equation 2 shall become negative; i.e., that

$$
\gamma \mathrm{e}^{\alpha \delta}>1
$$

Then the current will grow spontaneously and will result in a glow discharge at low pressures or an arc discharge at higher pressures. This breakdown can occur in less than $10^{-5}$ seconds and will be self-sustaining as long as the inequality in Equation 3 is satisfied.

The above transition from the Townsend discharge to a self-sustaining breakdown, in its broadest meaning, defines an electric spark. Sometimes the breakdown is noisy, destructive, and explosive in character because of the amount of energy liberated in a small volume.

The character of the growth and development of a spark has been the subject of much study over the years. For low pressure and short gaps, the spark mechanism is that indicated by Townsend's breakdown criterion. The time intervals observed for the development of the spark in such low-pressure breakdowns correspond to those calculated for the movement of slow ions across the gap and the buildup of space charges.

Difficulties are encountered when this theory is applied to sparks at atmospheric pressure. The Townsend theory does not apply to the common spark with which everyone is familiar. The time for these atmospheric sparks to build up is of the order of $10^{-7}$ seconds-not enough time by a factor of 100 for positive ions to cross a centimeter gap and build up a space charge.

The mechanism of these sparks as explained by Loeb (Reference 2) is as follows. A photoelectron starts across the gap from the cathode in the high field. When it arrives at the anode, it has created a large number of electrons and ions. The rapidly moving electrons are swept into the anode leaving behind most of the positive ions in a very small conical volume.* The large positive ion density produces an additional space charge field of value $\mathrm{E}_{1}$ at its tip. This adds vectorially to the existing field $\mathrm{E}$ in the gap. Photon emission caused by the radiation accompanying the creation of $10^{7}$ ions is very intense.

*At atmospheric pressure this volume is $0.4 \mathrm{~mm}$ long and perhaps $0.1 \mathrm{~mm}$ or less in radius and contains $10^{7}$ ions. 
In a mixed gas, such as air, or any relatively impure gas, the photons created can ionize some of the molecules in the gas. Absorption is rapid for the wavelengths that ionize, so that the photoionization is produced in a relatively confined volume near the tip of the concentration of ions. As a result, around the tip of the space charge, photoelectrons are created in a field of $E+E_{1}$. These create more ions as they are drawn toward the tip. The newly created electrons move along the positive space charge to the anode and by this mechanism the streamer advances in length into the gas. This process continues until the fields $\mathrm{E}+\mathrm{E}_{1}$ get too weak to advance the streamer or until the streamer constitutes an effective conducting channel between the electrodes. As it approaches the cathode it seriously distorts the imposed field E. A burst of photoelectrons from the cathode, and the electrons they subsequently produce by ionization, bridge the space between the cathode and the space charge, and a heavy current wave runs up the channel. This causes the intense ionization illumination, and heat that characterize the spark. In a sense, the positive streamer acts like an initial rip in a mechanically stressed medium. As it advances, it concentrates the electrical stress in a narrow region leading to easy breakdown.

Streamer breakdown occurs only at pressures and under conditions where intense photoionization can occur near the space charge. As pressures go down and $\mathrm{E} / \mathrm{p}$ increases, the photoelectric ionization decreases and actions such as the release of electrons by impact at the cathode come into play. Thus, at some lower pressure, for a given gap length, sparks will pass more easily by Townsend's mechanism than by streamer. The pressure where this occurs is not certain. It probably occurs below 100 torr in air for a 1-cm gap. All very long sparks such as high-tension flashover and lightning operate by streamer mechanism or by modifications of streamer mechanism.

The glow discharge occurs in the lower pressure ranges. The characteristics of a glow discharge are shown in Figure 4. The walls of the tube play a significant role in the behavior of the discharge. Currents in the glow discharges range from microamperes to amperes, but are usually in the milliampere range. Glow discharges require high potentials in comparison to ares, and current densities are low. As potentials increase, the glow may go over to the arc due to heating of the cathode by positive ion bombardment and consequent release of material from the cathode. Usually the change from glow to arc is very abrupt.

The glow discharge is characterized by a definite luminous structure. The cathode will usually be covered by a glow composed of radiation arising from positive ions undergoing recombination. This glow, called the cathode glow, generally obscures the Aston dark space which is immediately next to the 


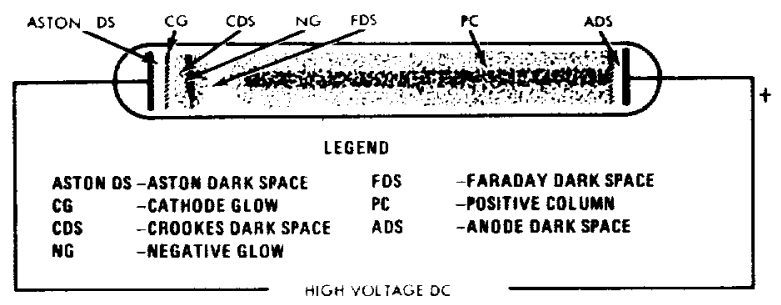

Figure 4 - A typical glow discharge.

cathode. The glow may be a small patch on the cathode or it may cover the whole front of the cathode surface or even extend slightly to the rear. When the glowing area on the cathode is small, the current density in the glow and applied potential remain nearly constant. If the current is increased by decreasing the external resistance in the circuit, the glowing area on the surface of the cathode increases, but the current density remains the same. As the glow covers the surface, a further current increase requires an increase in applied potential. The cathode fall of potential is a sharp potential decrease in the region of the cathode and is present in all discharge conditions. It results from a positive ion space charge in the gas. The potential fall ranges from some tens of volts to over 1000 volts in some gases.

The Crookes dark space is the visual evidence of the cathode fall. It lies near the cathode at relatively low pressures. Its length varies from some centimeters at very low pressures to fractions of a millimeter near 10 torr. It is the region over which the cathode fall occurs and is only relatively dark. It is populated by positive ions moving toward the cathode. The electrons from the cathode, freed by the impact of these ions, are accelerated quickly. They move through the dark space at high velocities and have little chance of causing ionization in this region. This makes the space relatively dark.

Next to the Crookes dark space is the negative glow. The fast electrons cause intense ionization accompanied by much radiation, particularly in the ultraviolet region. In the region of the glow farthest from the cathode, the potential may become negative relative to the edge of the positive space charge because of the accumulation of electrons and loss of positive ions.

Beyond the negative glow, there is a fainter dark space known as the Faraday dark space. In this region electrons are accelerated from the negative glow region into the long luminous positive column. The Faraday dark space results from the intense space charge produced in the negative glow. Its lack of luminosity arises because the electrons from the negative glow have not attained sufficient energy to cause ionization. This dark space is very noticeable at higher pressure and moves toward the cathode as the pressure is increased. 
The positive column is the luminous column in neon signs or any glow discharge. This column is essentially a gaseous conducting channel. The potential increases slightly along the column to the anode. The field is just strong enough to cause enough ionization to keep the channel conducting, balancing losses of ions and electrons. The potential gradient in the column is small. The positive column can be lengthened by increasing the applied potential.

At the anode there is another sharp increase in field strength and potential termed the anode fall of potential. It accelerates the electrons from the positive column in their last ionizing paths to give the needed positive ions for maintaining the column by ionization in front of the anode. This last ionization creates the anode glow. Sometimes observable following the anode glow is an anode dark space.

In summary, the glow discharge is maintained by electrons fed into the column from the cathode end. The cathode electrons are generated by an electrode emission mechanism involving both positive ion impact and photons. In the positive column there is much light from atoms and molecules excited by slower electrons. Some recombination takes place in the column. However, most of the loss of ions and electrons in the column comes from diffusion to the walls. The production of ions in the column just makes up for these losses and keeps the column conducting. The color of the light emitted depends on the gas involved and energy of the electrons in the column. Much of the emitted light is ultraviolet and is converted to heat at the walls. For further information on the glow discharge, see References 3,4 , and 5 .

The arc discharge operates at lower potentials and higher currents than the glow discharge. It can do this since its mechanism for electron generation does not require ion bombardment and the accompanying high fields. Instead, the arc utilizes its high current densities to furnish electrons at much lower potentials. The generally accepted theory is that the high current density of the arc heats the cathode to a temperature sufficient for the thermionic emission of electrons or the evolution of easily ionized gases.

The potential change in the vicinity of the cathode in an arc is only 10 to 20 volts, but the typical current density is of the order of $10^{4}$ amperes per square centimeter. Since a large number of charge carriers are needed to support the high currents, most arcs take place at pressures above 10 torr. Arcs operate on alternating or direct current. The column of the arc is selfcontained without walls since the higher pressures reduce diffusive losses. The current in an arc is controlled by the external resistance of the circuit rather than by its own impedance. The external resistance and the condition 
in the discharge region determine whether a given spark will end in a glow, an arc, or no continuing current. For more information regarding the arc discharge see Reference 3.

The term corona is often understood to encompass all forms of low current gas discharge. In its strictest usage, corona applies to a partial breakdown caused by high fields at one electrode, but no current bridges the gap between the electrodes.

The behavior of corona discharges is not basically different from the discharges already discussed. If there is a strong electric field, free electrons released in the gas near the positive electrode move toward it. The electrons gain energy rapidly from the intense electric field and they produce many ions in a small volume near the anode. When the ions get sufficiently numerous, a positive streamer moves outward from the electrode. The electrons are picked up by the electrode, and the field weakens when a positive space charge builds up. This gives rise to a tendency for the positive corona to choke itself off. As the fields get higher, the streamers propagate further toward the cathode. At this point, in place of the occasional short streamer and the flickering bright blue film (ionized air) at the anode, longer streamers project outwards. Such streamers give a characteristic brushlike appearance. In air they are bright blue in color. The ions left behind, which produce the space charge, show general purple haze characteristics of a low-voltage discharge in air. The brush discharge is a positive corona with a purple haze pierced by large numbers of bright blue streamers.

At increased potentials the streamers will propagate to the nearest cathode. When they reach the cathode with enough intensity a spark results, followed by a glow, are, or extinction depending on the discharge conditions. In very pure $\mathrm{H}_{2}$ and $\mathrm{N}_{2}$, streamers do not readily form since photoionization in these gases is poor. In very pure argon, they form so easily that the first streamer leads to spark breakdown. They are responsible for the noisy corona static which interferes with radio reception in airplane flight and near power lines.

At the negative electrode, electrons are released by ultraviolet light, or by positive ion impact. They gain energy rapidly because of the strong electric field, as do the electrons in the Crookes dark space in the glow discharge. They collide with the gas molecules and produce ions. This region is like a negative glow, with the Crookes dark space against the cathode. The electrons move beyond the "negative glow" and are accelerated toward the anode. Positive ions from the "negative glow" are drawn to the cathode and release new electrons on impact. In addition to these electrons, electrons are released from the cathode by the photoelectric effect and by photoionization of the gas. 
In electronegative gases, where electrons can attach to molecules to form slow-moving negative ions, the ions formed near the cathode end of the positive column, or even in the glow itself, will choke off the sustained discharge, and until the field sweeps these negative ions away, the discharge cannot continue. In this manner, an irregular intermittent negative corona may be obtained. If a plentiful source of electrons is available at the cathode to again initiate the discharge as soon as the negative ions are cleared away, the discharge will behave like a relaxation oscillator. Breakdown from a negative point occurs when a positive streamer mechanism can form in the gas and propagate outward.

Both positive and negative coronas have been observed about pointed conductors during high electrical stress, e.g., in thundershowers or about high tension lines. They were called St. Elmo's fire. If the time rate of change of increasing potential gradients is not too great, the corona may protect the circuit against total breakdown by space charge production. However, coronas can generally be regarded as danger signs which indicate defective performance.

\section{Breakdown in Alternating Fields}

At sufficiently low ac frequencies, it is observed that breakdown occurs when the peak value of the ac voltage reaches the dc value predicted by the Paschen type curve. However, as the frequency is increased, a decrease in the sparking potential is observed when a certain critical frequency is exceeded. This behavior may be understood when we consider that if the period of the applied voltage is long compared to the transit times of the ions in the gap, the situation becomes comparable to de breakdown conditions. On the other hand, when the period becomes comparable to the transit time of the ions, field reversal may occur before these charges are collected at the electrodes, and their number density may increase in the gap resulting in a field distortion.

The value of the lowest critical frequency will be determined by the slowest moving species of charged particles. For the case where only electrons and positive ions are present, the motion of ions determines the lowest critical frequency. For a 1-cm gap in air at one atmosphere, for example, the sparking potential remains constant up to about $20 \mathrm{kHz}$, above which it decreases steadily to a value about 15 percent lower. With a smaller gap the transit time is reduced and the critical frequency is increased.

As the frequency is increased more, a second critical frequency is reached at which the electrons are no longer collected at the anode during each halfcycle. This occurs in the range of 5 to $15 \mathrm{MHz}$ for a 1-cm gap in air at atmospheric pressure. The resulting decrease in sparking potential amounts to 30 to 40 percent. This decrease cannot be ascribed to the buildup of space charge 
since one electron is produced for each positive ion and both tend to accumulate in the gap. The drop in breakdown voltage occurs because an electron, uncollected at the anode after a half-cycle, will traverse the gap again during the next half-cycle. The amount of ionization produced by each electron is thus greatly increased. As the frequency is further increased, practically none of the electrons are collected. Electrons are now lost only by diffusion to the container walls and electrodes, and by recombination with positive ions. In electronegative gases, attachment of electrons to neutral molecules may also be important. In this frequency range, the criterion for breakdown is determined by the balance of the rate of electron generation with the rate of loss by diffusion processes. This is the diffusion theory of breakdown. Where this mechanism applies, the breakdown voltage is frequency independent.

Throughout the lower range of frequencies, where the positive-ion space charge still plays a dominant role, secondary electron production is of prime importance; the Townsend theory of breakdown still applies. As the frequency is increased to the range where the diffusion theory applies, secondary electron production is of little importance, and the breakdown is determined by the generation rate caused by primary processes and by the losses from diffusion. This theory is not applicable when the mean free path of electrons becomes of the same order as the dimensions of the physical system under consideration where secondary processes become important again.

A short summary of high frequency and microwave breakdown in gases, the governing equations, and typical Paschen-like curves are presented here, with more detailed treatments being given in References 6 and 7 .

Breakdown in high-frequency gas discharges is controlled by primary ionization caused by electron motion. When the gain of electrons from ionization equals the electron losses to diffusion, recombination, and attachment, breakdown occurs. The simple case of losses by diffusion only will be considered first.

For a region bounded by walls that absorb electrons, breakdown occurs when

$$
\nu_{i}=\mathrm{D}(\pi / \mathrm{L})^{2}
$$

where $v_{\mathrm{i}}$ is the ionization field frequency, $\mathrm{D}$ is the diffusion coefficient for electrons, and $L$ is the distance between the walls. If we write $v_{i} / D=(\pi / L)^{2}=$ $1 / \Lambda^{2}$, for parallel plates, the quantity $\Lambda$ is called the characteristic diffusion length. A useful example is that of a cylinder of height $L$ and radius $R$, for which $(1 / \Lambda)^{2}=(\pi / L)^{2}+(2.4 / R)^{2}$, where the diffusion to the ends is given by the term containing $L$ and diffusion to the walls is given by the term containing $R$. 
Figure 5 -High frequency $(\sim 100 \mathrm{MHz})$ gas breakdown characteristics.

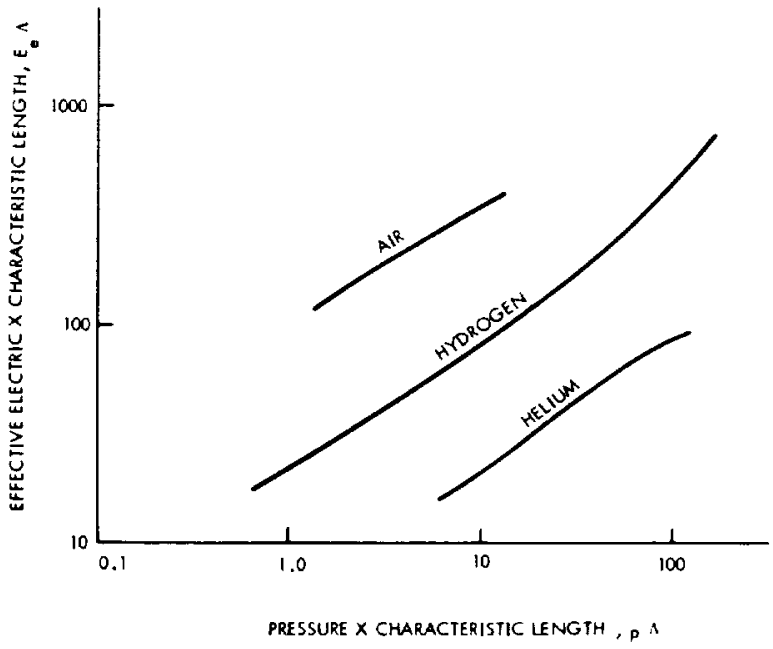

Since the high-frequency breakdown is a primary ionization phenomenon, it is related to the first Townsend coefficient, $\alpha$. If we define $\eta$ as $\alpha / E$, we can express it in terms of an "ionization collision frequency, " $v_{\mathrm{i}}$. This has the form

$$
\eta=\nu_{i} / \mu \mathrm{E}^{2}
$$

where $\mu$ is the electron mobility.

A Paschen-type curve can be developed for high frequency breakdown. The coordinates are $\mathrm{E}_{e} \Lambda$ and $\mathrm{P} \Lambda$ as shown in Figure 5, which shows how air, hydrogen, and helium behave at high frequencies; $\mathrm{E}_{\mathrm{e}}$ is the effective electric field given by $\mathrm{E}_{\mathrm{e}}^{2}=\mathrm{E}^{2} v_{\mathrm{i}}^{2} /\left(v_{\mathrm{i}}^{2}+\omega^{2}\right)$, w being the field frequency.

It should be noted that the greater the electrode spacing the easier it is to cause breakdown for a given field strength at high frequencies.

Secondary emission of electrons by direct bombardment of the walls can cause high frequency breakdown at pressures, fields, and frequencies where the discharge is not controlled by diffusion. For this type of breakdown, known as multipacting, both the magnitude of the field and the phase of the electron motion with respect to the electric field are important. The condition most likely to result in breakdown occurs when electron motion is in phase with the electric field. The secondary electrons will then be accelerated across the gap. The electric field frequency must be of such a value that the electron transit time is equal to one-half cycle time of the field. Under those circumstances large numbers of electrons exist in the space between the walls and are caused to flow back and forth by the alternating field. 


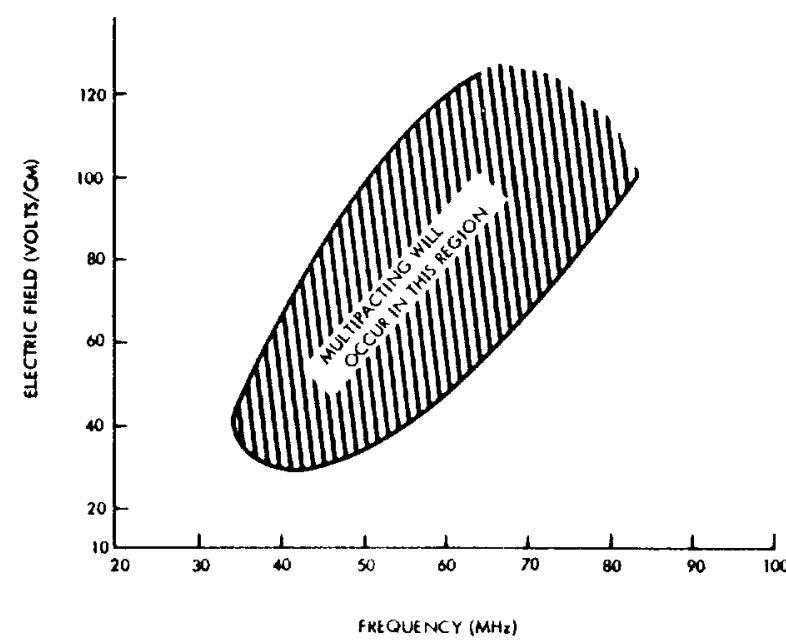

Figure $6 \quad$... The values of electric field and frequency for which multipacting may occur.

The presence of such a cloud of electrons will cause breakdown over a fairly broad range of frequencies and fields. For a given frequency, breakdown is possible in a region limited by two values of the field corresponding to too little or too much acceleration of the electrons leading to a loss of the proper phase relations. This type of breakdown relies upon secondary electron emission from walls; it is independent of the type of gas but highly dependent upon the nature of walls containing the discharge. This breakdown mechanism is normally found at low pressures where the mean free path is long and ionization in the gas is unlikely.

Figure 6 (Reference 6 ) shows the breakdown region in hydrogen at a pressure of less than 0.1 millitorr as a function of peak electric field versus frequency for a $3-\mathrm{cm}$ electrode separation.

\section{Emission of Electrons and lons by Solids}

A phenomenon closely associated with various gas discharges is the emission of charged particles by solids such as insulating walls and metal electrodes. The emission of electrons is one of the factors that determines whether or not a gas discharge is to be self-sustaining, and it is therefore of considerable interest in the study of conduction of electricity through gases. Electron emission is greatly influenced by the condition of the emitting surface. It may result from high temperatures (thermonic emission), electron bombardment, positive ion bombardment, or bombardment by metastable atoms; or it may arise from high fields at the surface, chemical effects and photoemission. It is beyond the scope of this paper to discuss these effects in detail. For information beyond the remarks below see the literature (Reference i). 
Thermionic Emission. Thermionic emission becomes appreciable only when the solids are very hot, at incandescent temperatures in most cases. Materials at such temperatures are not ordinarily encountered in spacecraft high-voltage systems. When an electrical breakdown causes local heating, thermonic emission may become great enough to add to the general catastrophe.

Electron Bombardment. When solids are bombarded with electrons, the electrons traveling away from the surface include incident electrons which have rebounded with a range of energies dependent upon the number of inelastic collisions they have experienced, and the number of electrons which have been ejected from the solid by the incoming electrons. The latter are called secondary electrons, and with a clean metallic surface they begin to appear at primary energies on the order of 10 to 20 electron volts. As the energy increases to several hundred volts, efficiency of production of secondaries rises to about unity; i.e., one secondary is produced by each primary. Certain types of surface layers on metals can increase the yield of secondaries several hundredfold (Reference 2 and 3).

Heavy Particle Bombardment. The impact of heavier particles such as atoms and molecules, both ionized and neutral, also causes the ejection of electrons from solid surfaces. Under certain circumstances the heavier particles may also drive ions or neutral atoms, or even groups of atoms, out of the solid surface.

Photoelectric Emission. Photoelectric emission can also introduce electrons into the gas discharge region. The arrival of a sufficiently energetic photon can result in the ejection of an electron from the surface with a certain initial velocity which may be augmented by the effects of existing electric fields.

Field Emission. Electrons can also be released from a solid surface when it is cold, clean, and not irradiated, by the mechanism of field emission. If the electric field at a solid surface is greater than $5 \times 10^{5}$ volts per centimeter, electrons may be pulled out of the surface with consequent effects on gas discharge conditions. Five hundred thousand volts per centimeter seems enormous and unlikely to occur, but in the presence of sharp points or edges such potential gradients can and do occur with very moderate applied voltages. It is also possible for very high field strengths to occur as two parts of a mechanical switch approach each other in the process of closing. Thus breakdown can occur by field emission upon closing in addition to the familiar spark that occurs when the circuit is broken. 


\section{Electrical Breakdown in Liquids}

Since electrical breakdown in liquids is not very important in space applications, the discussion is limited to the following short summary and the general information contained in Table 1.

Voltage breakdown in liquids is influenced by contaminants. An applied electric field tends to sweep contaminants into the area of highest field strength. The increasing concentration of contaminants at the point of highest potential gradient eventually produces a "bridge" across which arcing can occur. Arcing produces decomposition products, which are themselves contaminants; and the local temperature rises, producing further deterioration. When voltage stress is removed, the contaminants can diffuse out of the area of breakdown and, if the total volume is great enough, be sufficiently diluted to permit the system to operate satisfactorily, at least for a time.

\section{Electrical Breakdown in Solids}

Electrical breakdown characteristics of solid materials are particularly important in the design and fabrication of spacecraft systems because solids are widely used to prevent undesired electrical discharges. Insulating sleeves on wires, solid potting compounds, and conformal coatings are examples of such applications. If the materials used exhibit breakdown modes or progressive failure in a space environment which are not apparent in room-ambient testing, disastrous failures may occur in flight.

There are several processes which will generate heat in a dielectric in an electric field. Three of the most important ones are: (1) the flow of ionic currents; (2) interaction of electronic currents with the lattice; and (3) the displacement of bound ions or dipoles in an ac field. The quantity of heat generated by one or more of these mechanisms increases with the applied voltage, and if a specimen cannot dissipate this internally generated heat at a rate equal to its generation rate, the temperature will rise. Electrical breakdown may then occur when the dielectric strength falls as a result of temperature increase. What is called thermal breakdown may occur if the material melts or decomposes as the temperature rises.

Thermal dielectric breakdown is avoided by the use of materials that can either stand elevated temperatures with little loss of dielectric properties, or experience minimal heat buildup. The first is accomplished by selecting 


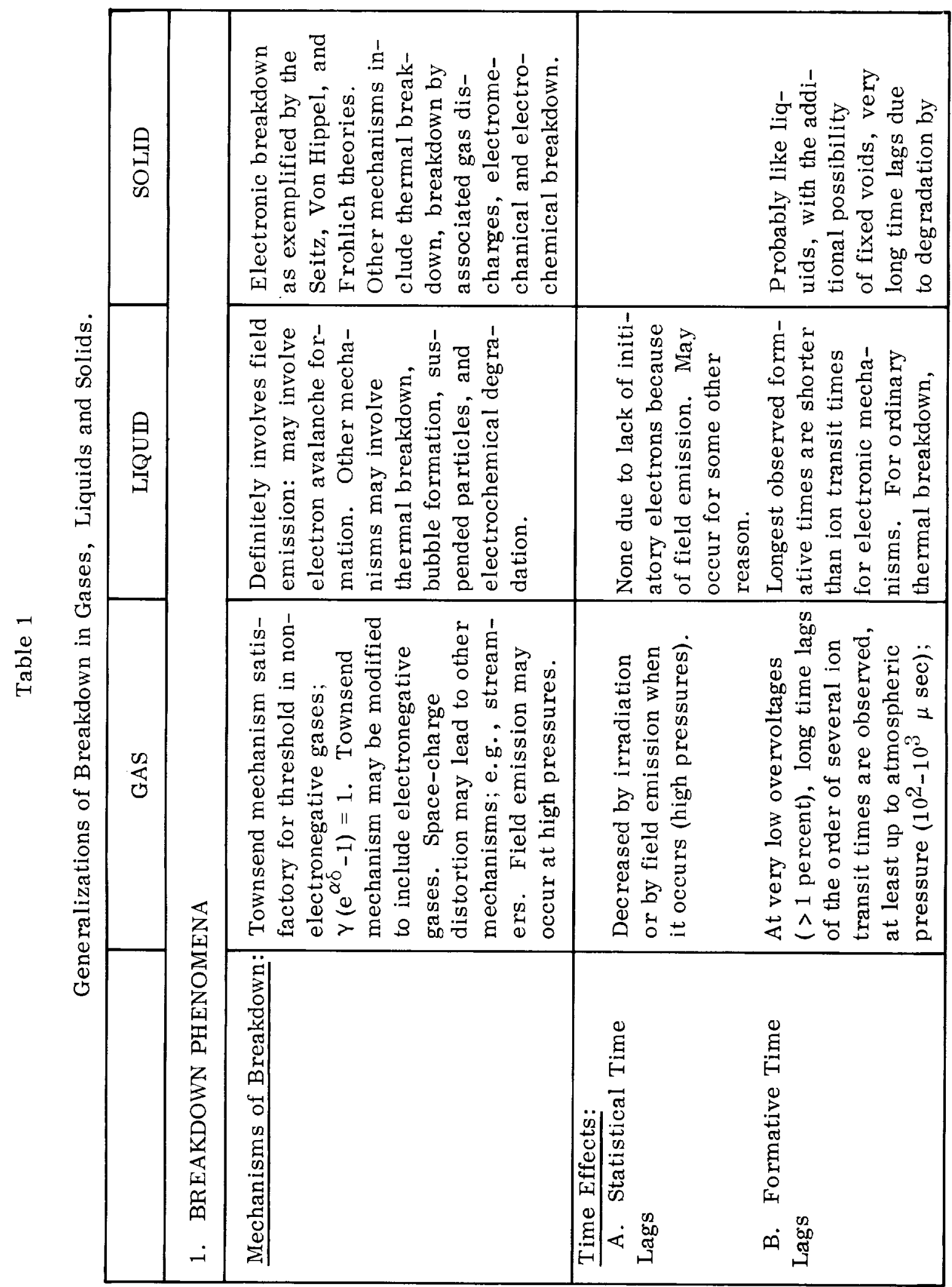




\begin{tabular}{|c|c|c|c|c|c|}
\hline$\underset{\wp}{\stackrel{\theta}{\ominus}}$ & & 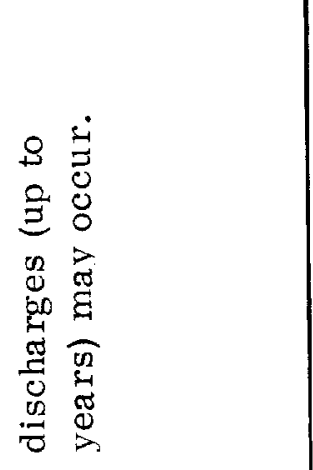 & & 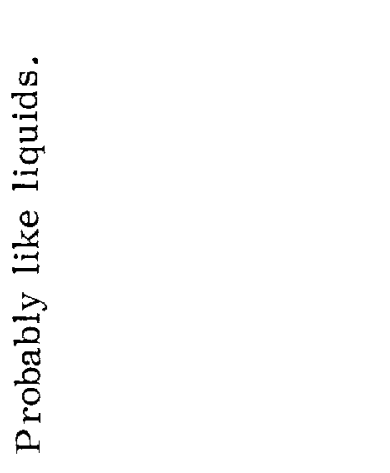 & 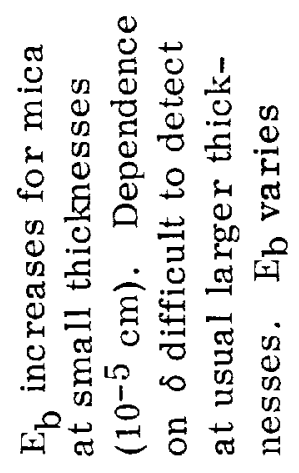 \\
\hline 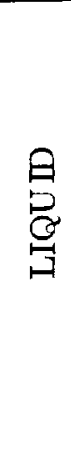 & & 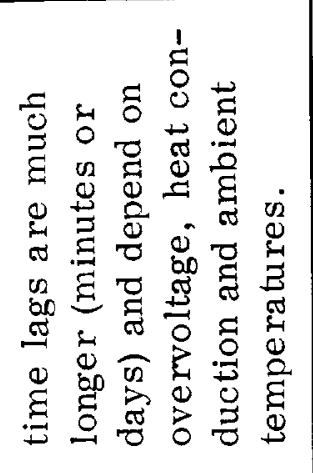 & & 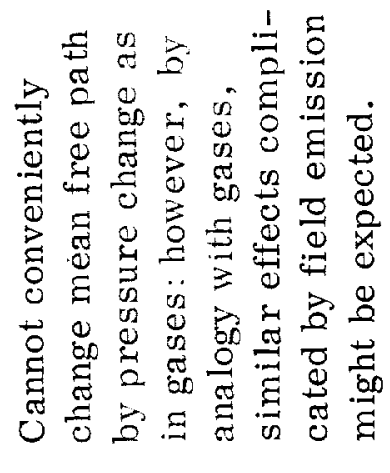 & 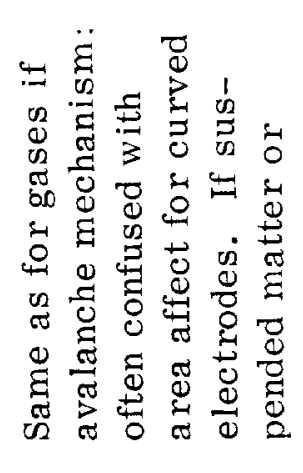 \\
\hline $\mathbb{J}_{0}^{\infty}$ & 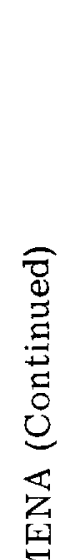 & 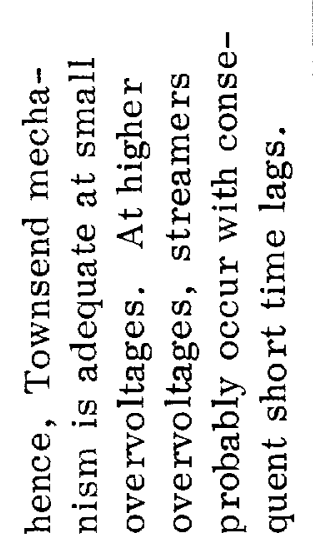 & 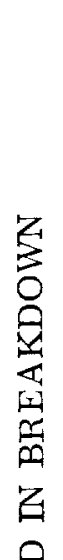 & 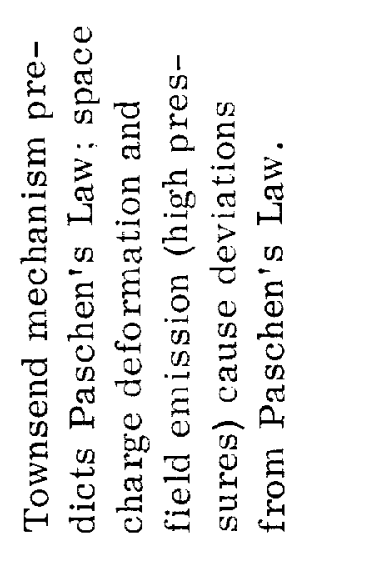 & 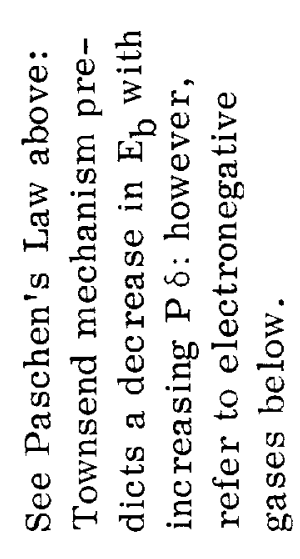 \\
\hline & 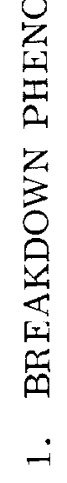 & & 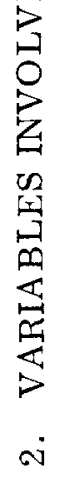 & 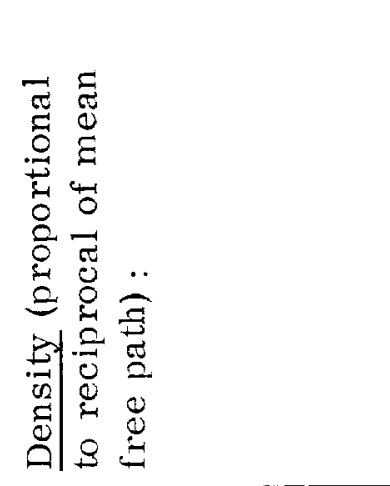 & \begin{tabular}{l|l}
0 \\
0 \\
0 \\
00 \\
0 \\
0 \\
0 \\
0 \\
0 \\
0 \\
0 \\
0 \\
0 \\
0 \\
0 \\
0 \\
1 \\
1
\end{tabular} \\
\hline
\end{tabular}




\begin{tabular}{|c|c|c|c|c|}
\hline $\begin{array}{l}\stackrel{\theta}{\beta} \\
\stackrel{0}{\circ}\end{array}$ & & 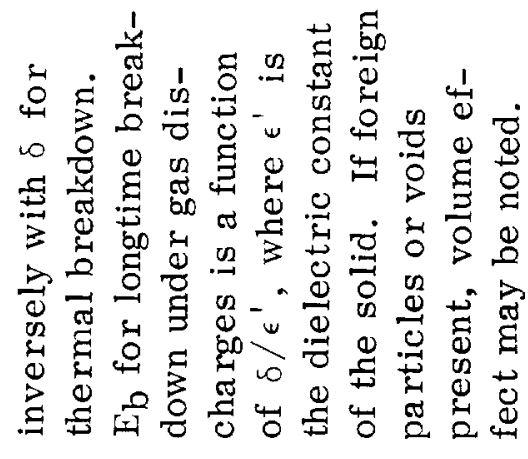 & 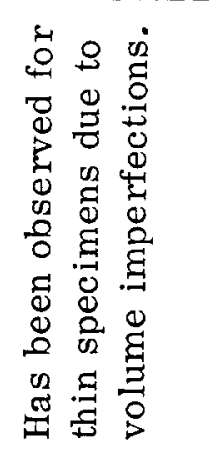 & 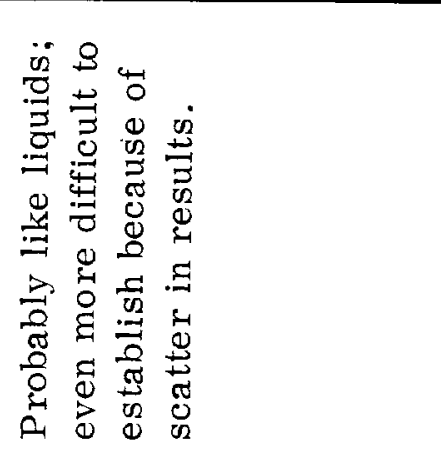 \\
\hline 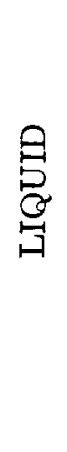 & & 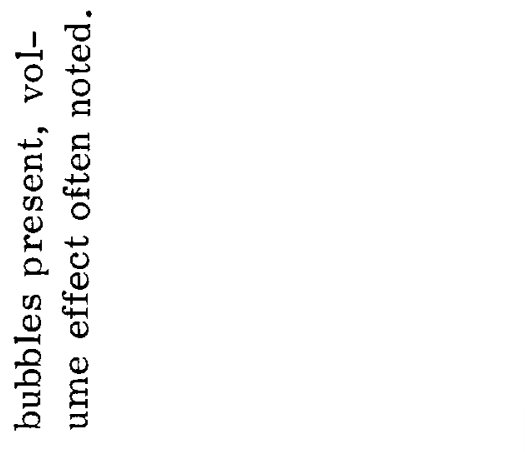 & 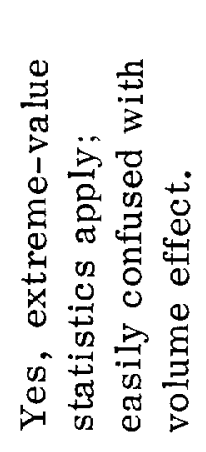 & 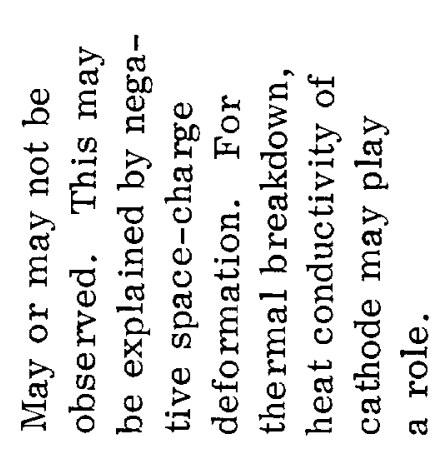 \\
\hline $\mathbb{\bigsqcup}_{0}^{\infty}$ & 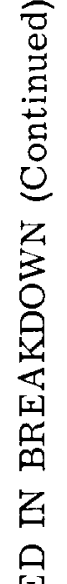 & & 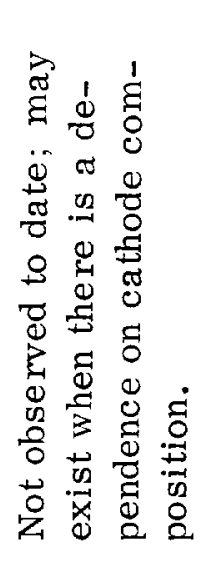 & 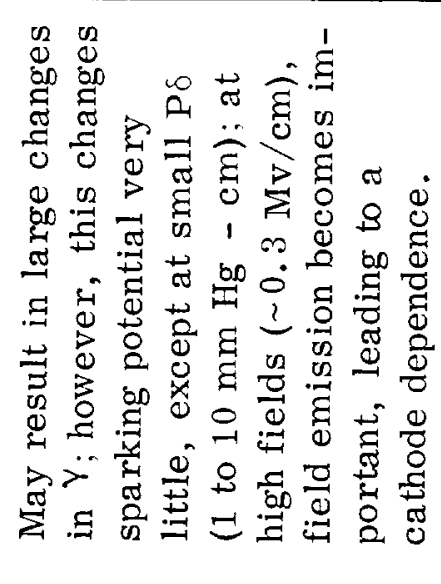 \\
\hline & 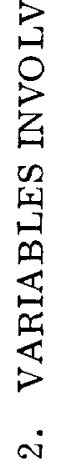 & & 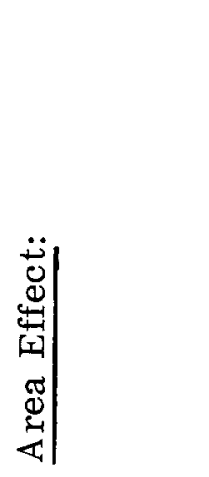 & 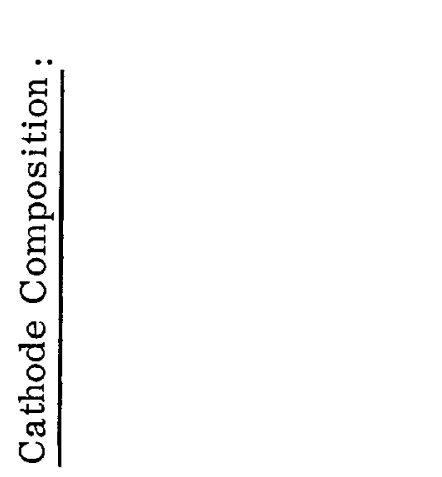 \\
\hline
\end{tabular}




\begin{tabular}{|c|c|c|c|c|c|}
\hline 目 & & 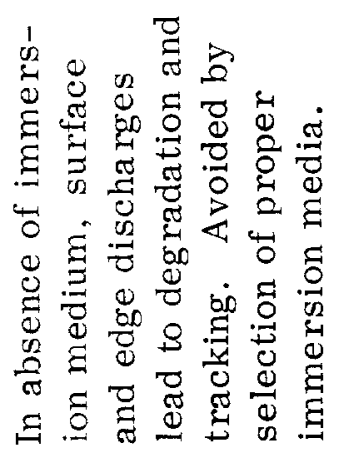 & & 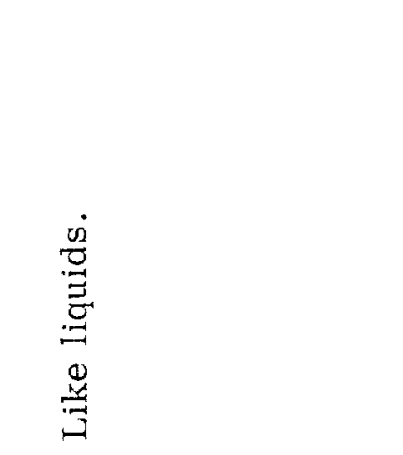 & 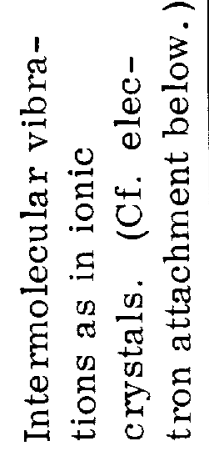 \\
\hline 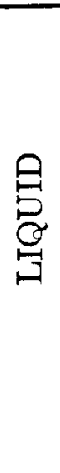 & & 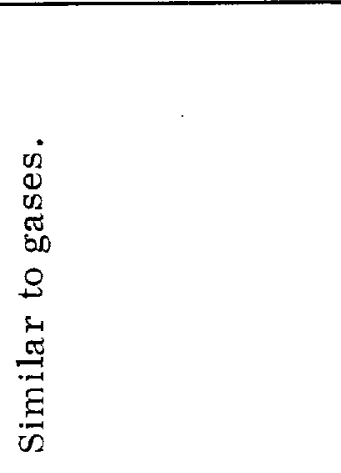 & & 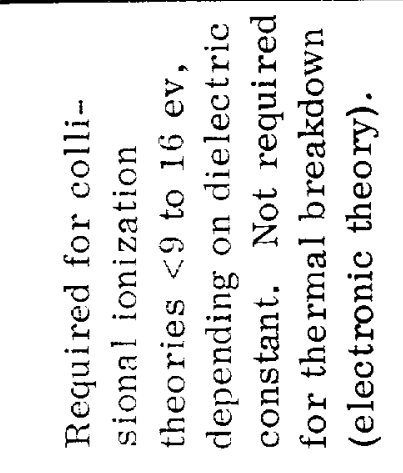 & 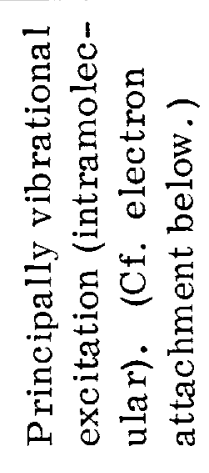 \\
\hline$\sum_{0}^{\infty}$ & 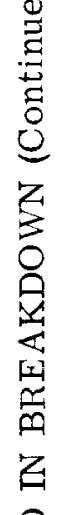 & 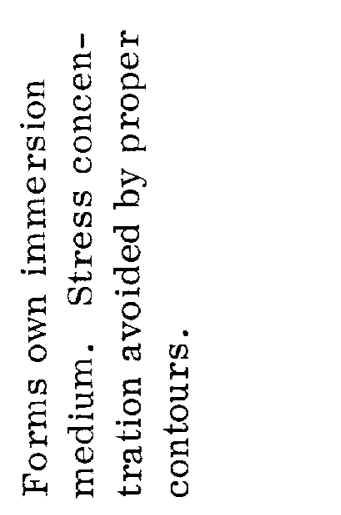 & 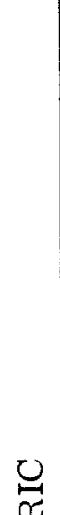 & 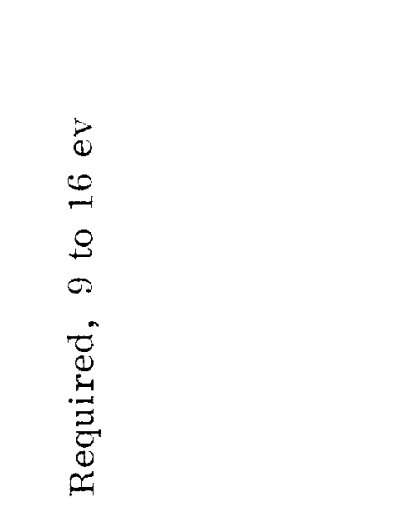 & 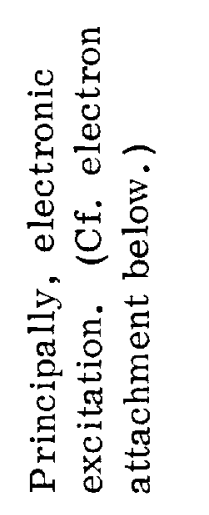 \\
\hline & 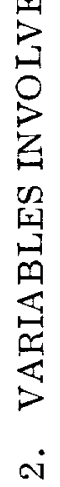 & 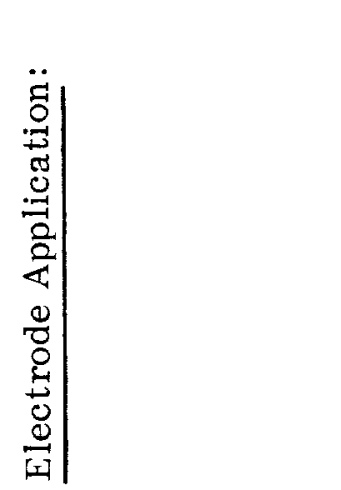 & 0 & 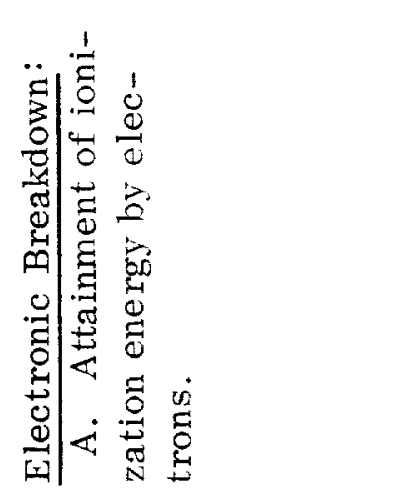 & 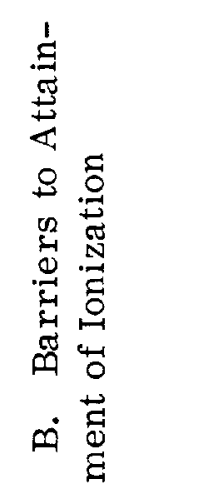 \\
\hline
\end{tabular}




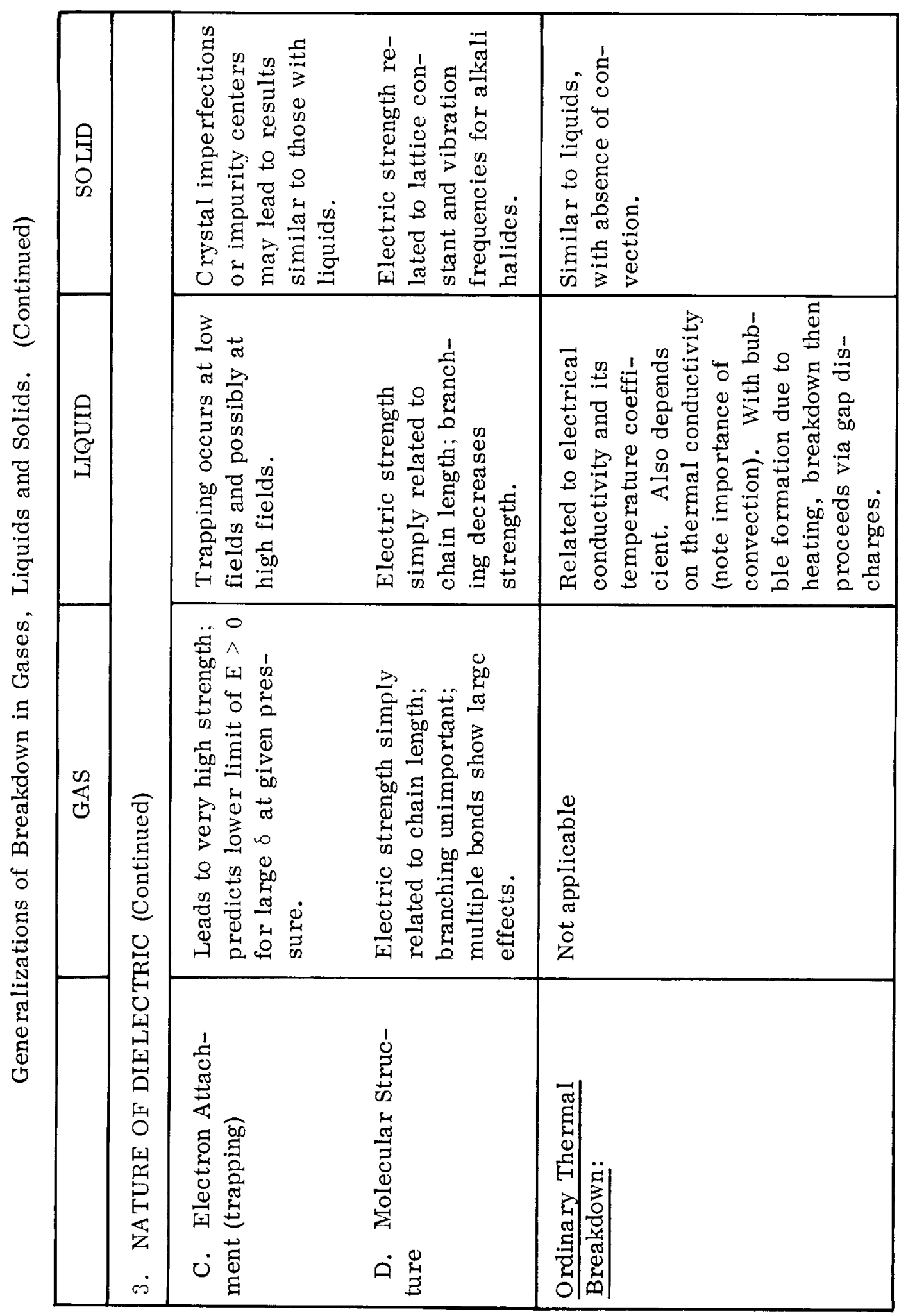




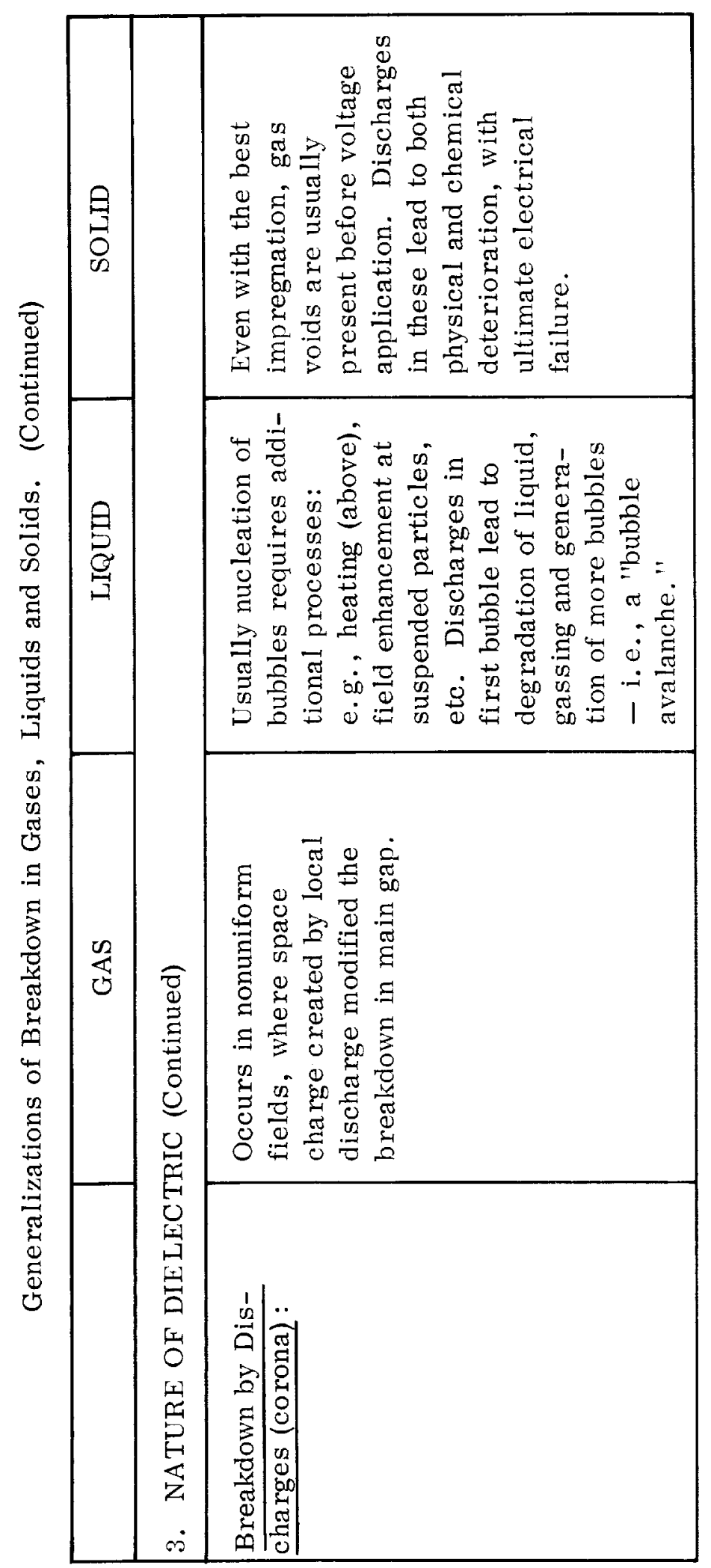


materials which are known to behave well in this regard. The second is achieved by selecting materials that do not absorb appreciable energy from the applied electric fields, and that provide for quick dissipation of heat that is produced by allowing radiation or conduction through the electrode structure or other mechanical parts.

\section{Breakdown Initiated by Gas Discharges}

The following material on breakdown initiated by gas discharges follows the development of Sharbaugh and Devins (Reference 8).

Even when one may eliminate the possibility of thermal mechanisms of failure by using "low-loss" materials, thin specimens with massive electrodes, and short durations of applied voltage, it is often found that the electric strength is much lower than that to be expected. It is usually lower for ac voltage than for dc voltage, and drops with increasing time of application of voltage. The rate at which it drops increases with increasing frequency of the applied ac voltage. Over a very long period samples may fail at stresses very little higher than would be required to break down an equivalent layer of air.

These phenomena are often the earmarks of breakdown initiated by gas discharges occuring either in voids in the solid or in the vicinity of electrodes on the surface. Such discharges chemically degrade the solid and result either in a gradual erosion until the intrinsic strength is exceeded in some spot, or lead to the production of species which raise the electrical losses to the point where thermal runaway is possible. Often, if mechanical forces are present, electrical failure may occur as a result of the degradation of mechanical properties by the discharges. For example, in many polymers, cross-linking reactions may cause embrittlement, with ultimate failure due to cracking.

Materials under mechanical strain may undergo "stress-cracking" or "ozone-cutting" owing to scission of polymer chains by electrical discharges or their byproducts.

Although it is relatively easy to delineate the processes by which the discharges may cause chemical reactions in the solid, it is very difficult to assess the true relative importance of each. The electrons and ions striking the solid surface have sufficient energy, kinetic or potential, to lead to rupture of chemical bonds by processes similar to those known to occur in radiation chemistry. The same is true of the ultraviolet light produced in the discharge. Depending upon the gas present in the discharge, various highly reactive atoms, free radicals, and electronically excited molecules are produced which can react with the solid. Finally, new molecules may be formed in the gas discharge which 

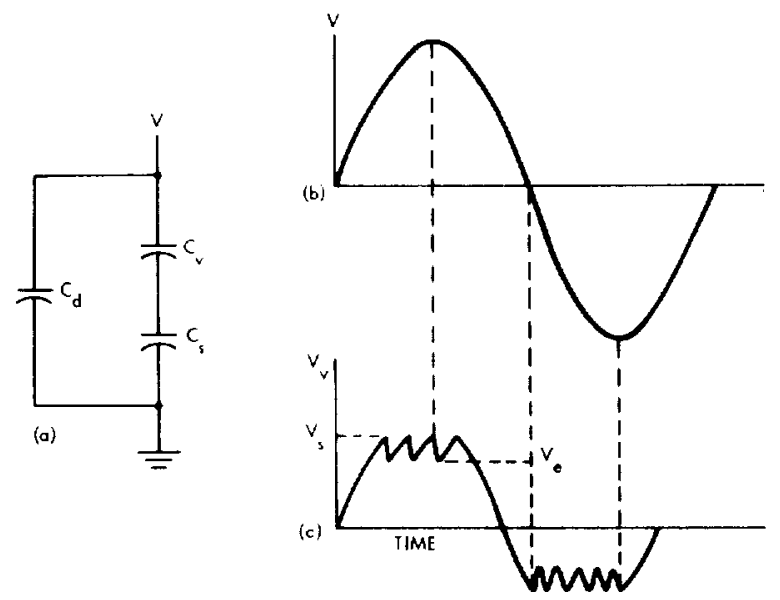

Figure 7 - (a) Equivalent circuit of a dielectric containing a void. (b) Time dependence of applied voltage, $\mathrm{V}$.

(c) Time dependence of void voltage, $\mathrm{Vv}$.

will react with the adjacent solid dielectric. In air, for example, highly reactive nitrogen oxides and ozone are produced.

Consider the model for a dielectric containing a void shown in Figure 7a. Here, $\mathrm{C}_{\mathrm{V}}$ represents the capacitance of a void in series with a solid dielectric whose capacity is $\mathrm{C}_{\mathrm{S}}$; and $\mathrm{C}_{\mathrm{d}}$ represents the capacitance of the remaining solid in parallel with the combination. If a sinusoidal voltage, $\mathrm{V}$, is now applied to the system, the voltage will divide capacitively between $\mathrm{C}_{\mathrm{V}}$ and $\mathrm{C}_{\mathrm{S}}$-provided that the electric strength of the gas in $\mathrm{C}_{\mathrm{v}}$ is not exceeded and no discharges occur. It has been shown that the electric strength of a gas is relatively independent of the nature of the electrode material. Even when the electrodes consist of a typical solid dielectric, the sparking potential, $V_{S}$, differs little from that for most metal electrodes. Thus when the voltage across the void, $\mathrm{V}_{\mathrm{v}}$ (Figure 7a), reaches $V_{S}$, breakdown will occur. Charge produced by ionization in the gas in $\mathrm{C}_{\mathrm{V}}$ will remain on the insulating surfaces of $\mathrm{C}_{\mathrm{V}}$ to lower its potential to $\mathrm{V}_{e}$, the extinction voltage for the discharge. At the same time, charge will flow from the external circuit (including $\mathrm{C}_{\mathrm{d}}$ if the external circuit impedance is sufficiently high) to raise the voltage across $\mathrm{C}_{\mathrm{S}}$. The discharge time is very short-perhaps $10^{-7}$ seconds-and can be related to the transit times of ions and electrons in the discharge.

After the fast discharge, the voltage on $\mathrm{C}_{\mathrm{V}}$ continues to rise at the sinusoidal rate until, after an increase to $\mathrm{V}_{\mathrm{S}}$, a further discharge can occur. These changes will continue in the neighborhood of the peak of the applied voltage, after which the voltage across $\mathrm{C}_{\mathrm{v}}$ will decrease and discharges will cease. The charge remaining on $\mathrm{C}_{\mathrm{v}}$ will augment the applied field on the next half-cycle, and a new series of discharges will begin at an earlier point. This may occur even at the zero of applied voltage, as shown in Figure $7 \mathrm{~b}$ and $7 \mathrm{c}$, 
or before or after the zero, depending upon the amount of charge deposited. This, in turn, is a function of how much the peak voltage exceeds the starting voltage in the first half-cycle. As a result, more discharges may occur on the second half-cycle. After several half-cycles, a steady state will, of course, be reached at which time the average charge transported on each half-cycle will be the same. At this time, if the peak applied voltage is increased, discharges will begin at a lower applied voltage, and the number of discharges per halfcycle will increase.

An interesting feature of this model is that it permits an explanation of the often-observed intermittent discharges that may appear for many cycles and then cease. Thus even if the peak applied voltage is somewhat below that required for sustained discharges, it may be augmented by the field due to charge deposition by a chance discharge (owing, for example, to a fluctuation in voltage) so that a further breakdown can occur. Discharges may then continue on each half-cycle, but, as a little reflection will show, at an earlier point on the wave for each cycle. Eventually, the sum of the applied voltage and that caused by depositied charge will fail to reach the sparking potential on a half-cycle, and discharges will cease. The phenomenon will be accentuated for low values of the extinction voltage $\mathrm{V}_{\mathrm{e}}$.

A number of studies have been made of the so-called "epochs" of the discharges on the voltage wave; and, on the basis of these observations, the general validity of the above simple model has been confirmed. Detailed considerations suggest, however, that some modifications are necessary; for example, that except for voids of very small cross-section, the whole void is probably not discharged. Only relatively small areas appear to be involved in each discharge, and it seems likely that the extinction voltage is low. As a result, sites once discharged tend to remain in such condition for a relatively long time, changing slowly as transverse leakage occurs.

For dc stresses, the model in Figure 7 a predicts that discharges can occur only as the voltage is raised. In practice, of course, $\mathrm{C}_{\mathrm{S}}$ will always possess a leakage resistance, $R_{S}$, effectively in parallel with it, so that the voltage across $C_{V}$ can build up again with a time constant $R_{S} C_{S}$. Thus, depending upon the magnitude of the applied voltage and this time constant, recurring discharges in voids will occur even with de voltages. For most good dielectrics, however, $\mathrm{R}_{\mathrm{S}} \mathrm{C}_{\mathrm{S}}$ is very long and damage due to discharges is usually much less with de than with ac of comparable stresses.

Although it is time-consuming, perhaps the most effective method for evaluating the effects of discharges on electrical insulation is the measurement of the time-to-failure as a function of applied voltage. The fact that such a curve 
appears to approach infinite life asymptotically to the discharge inception voltage is good evidence that failure is caused by discharges. The time required to establish such curves may be considerably reduced by increasing the frequency of the applied voltage. Provided that the normal ac losses are sufficiently low so as to avoid dielectric heating, the frequency may be raised to as high as $1 \mathrm{MHz}$ without obtaining appreciable general heating of the dielectric by the discharges. It has been found that the rate of damage due to discharges is accelerated proportionally to the increase in frequency. This is not unexpected when one notes that, according to the model of Figure $7 \mathrm{a}$, the total charge transferred by discharges per half-cycle is a function only of the difference between the peak applied voltage and the voltage at which discharges are initiated. Thus the total charge transferred per unit time should be proportional to the frequency of the applied voltage.

Discharges occurring at surfaces may lead to an important phenomenon often referred to as high-voltage creepage. This may occur when relatively high stresses are present and tangential to the surface of the dielectric-as, for example, when two sharp-edged electrodes are place on a sheet of insulating material. If the voltage applied is insufficient to cause immediate breakdown, but high enough to cause discharges at the sharp edges of the electrodes, gradual degradation of the dielectric in the region of the discharges may occur. These regions may become partially conducting owing to carbonization or oxidation followed by uptake of moisture. Such conducting areas may thus ultimately constitute extensions of the electrodes and the discharges will "creep" over the dielectric surfaces. Eventually, the conducting areas may meet, and the system will fail by a power arc. Even though a system is initially free of discharges, contamination of the surfaces with dust and moisture may often cause local high gradients and accompanying discharges, leading eventually to creepage and failure.

\section{Summary of Electrical Breakdown Phenomena}

Table 1 (Reference 8 ) will serve as a summary to this section on breakdown phenomena. 


\section{DESIGN PRINCIPLES}

\section{Approach}

Three design aims are crucial for success in building and using high voltage systems for space flight:

a. Design to avoid breakdown, i.e., use components, materials, and configurations that minimize the chances of a voltage breakdown.

b. Design so that electrical breakdowns such as corona, glow discharges, arcs, and sparks will not be catastrophic to any equipment or instrumentation on the spacecraft.

c. Design to provide for frequent voltage-on tests at any appropriate step during fabrication, assembly, and integration of the high voltage systems.

\section{Designing to Avoid Breakdown}

\section{Geometrical Considerations}

a. Sharp Edges and Sharp Points. Sharp edges and sharp points must be scrupulously avoided. The presence of a sharp point produces a high electric field, or electrical stress, even when the applied voltage is quite moderate. For example, consider a rounded electrode with a $1-\mathrm{cm}$ radius of curvature with applied potentials such that the electrical field near the electrode is $200 \mathrm{volts} / \mathrm{cm}$. If the position is kept fixed and the radius of curvature is reduced to $1 \mathrm{~mm}$, the electric field becomes 20,000 volts $/ \mathrm{cm}$; if the radius is reduced to $0.1 \mathrm{~mm}$, the field strength near the electrode is $2,000,000$ volts $/ \mathrm{cm}$-and this without any change in position or applied voltage. Edges, as contrasted to points or spheres, will also produce very high localized fields if they are sharp or have small radii of curvature. As was pointed out earlier, high fields lead to electrical breakdown by accelerating accidental electrons and ions, and by producing field emission of electrons that contribute further to electrical breakdown.

Not only must electrode shape be carefully controlled, but also the character of the surface finish is critical. Local surface irregularities such as pits and blowholes produce local field concentrations with consequent liability to electrical breakdown. Therefore, the surface finish should be as smooth as possible. 


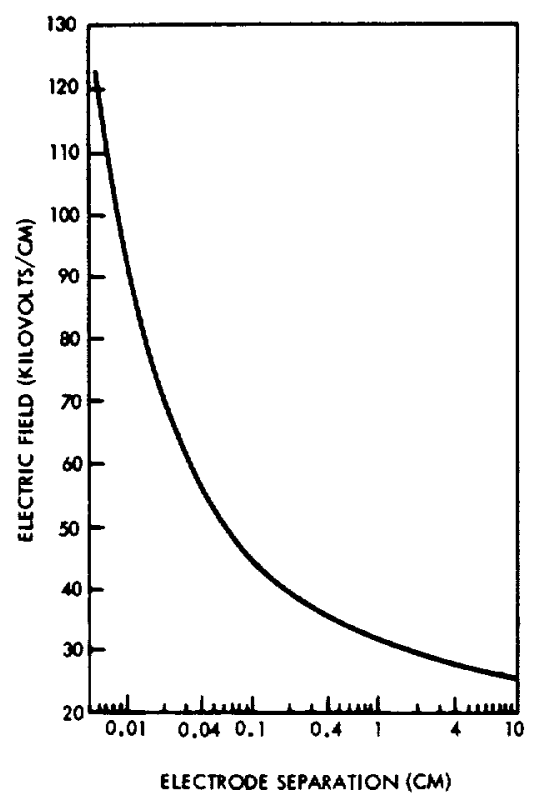

Figure 8 --Breakdown voltage gradient versus electrode separation in air at atmospheric pressure (Reference 9).

b. Spacing of Components. The spacing of components which vary greatly in voltage level must be considered along with the gas conditions that will exist near the equipment during operation. At atmospheric pressure, with dry gas, breakdown is to be expected if the electric field exceeds the values shown in Figure 8. At pressures of $10^{-4}$ torr and lower, electric fields approximately 20 times greater are required to cause breakdown on electrode separation of $1 \mathrm{~cm}$. At intermediate pressures, one must be guided by Paschen's curve, and at the minimum of that curve, an electric field as small as 100 volts $/ \mathrm{cm}$ may produce a breakdown as noted earlier. It is important to remember that some gases, particularly helium, neon, argon, krypton, and xenon exhibit breakdown at sea level atmospheric pressures at moderate applied fields. The use of these gases is not likely to prevent voltage breakdown in a system.

c. Multipacting. Another matter to be taken into account in planning the location of parts of the system is multipacting. To avoid this effect, electrode spacing, frequency, and applied voltage must all be considered. The general characteristics of the relation among these parameters (Reference 10) are shown in Figure 9, where the peak voltage is the ordinate while the product of frequency and electrode separation is the abscissa. This is a typical plot for parallel plane electrodes. The scale at the top is electrode separation divided by wavelength, both in centimeters. The regions indicated by $1 / 2,3 / 2,5 / 2$ are the regions within which multipacting can occur. The half integral values refer to half-cycle modes of oscillation. For example, the transit time between 
Figure 9-Possible regions of multipacting between parallel plates (Reference 10).

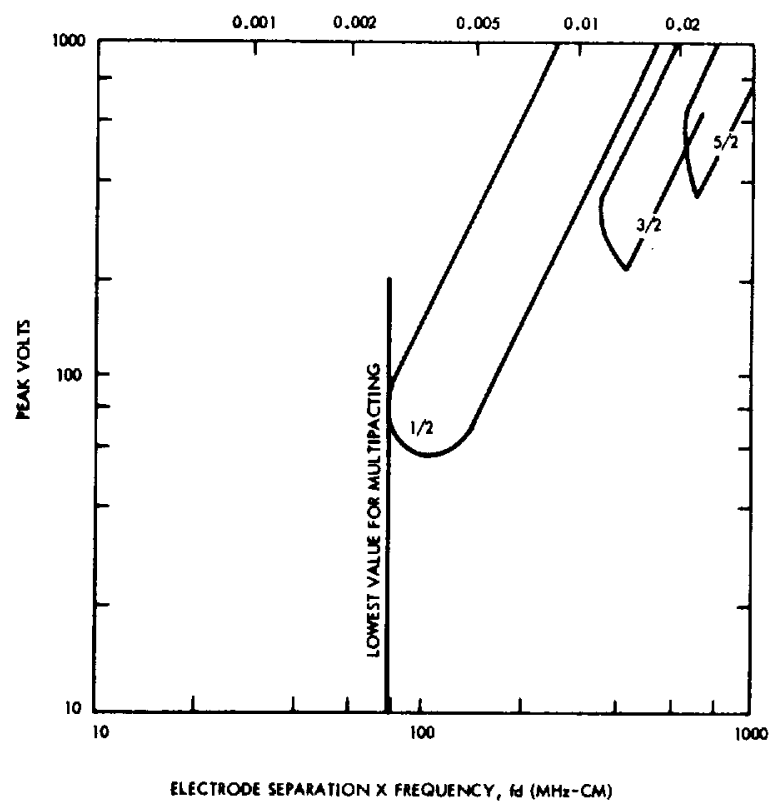

electrodes, for electrons, is one half-cycle for the area marked $1 / 2$. Such a chart cannot be relied on for design data, however, because the values depend on many factors, including number of electrodes, electrode material, surface conditioning of electrodes, and geometry (e.g., parallel plate, coaxial, etc.). The interaction of these factors is only known empirically, so the designer is forced to test every design at various stages of fabrication to be certain that multipacting is not occurring. It should be noted that multipacting is not a gas discharge, but it is an electrical phenomenon that can degrade system performance; under certain conditions multipacting will drive gas off solid surfaces in sufficient volume to cause the familiar gas discharge breakdown.

Some design features which have been found to give relief from multipaction are (Reference 11):

1. The insertion of foamed dielectric material between the electrodes

2. Treatment of electrode surfaces to change their secondary emission characteristics

3. The application of dc bias to the electrodes to suppress secondary electron emission

4. Pressurization of the space between the electrodes 
5. The selection of electrical and mechanical dimensions to reduce the likelihood of multipaction

Usually one or more of the above preventive measures will be found effective.

\section{Shiclding and Enclosures}

a. Insulation and Shielding. The choice of insulation for any wires carrying high voltage has an important influence on the prevention of breakdown problems. In general, the voltage rating for the insulation in spacecraft should be substantially higher than that required for ground-based operation of the same equipment. It is customary to require a rating twice as high for ground operation, and not unusual to require ratings five times higher. In addition to a high breakdown rating, the insulation must fit snugly onto the conductors and be free of voids both within the insulation and between the insulation and the conductor. This is necessary to prevent trapping of gas between insulation and conductor.

Gas pockets in insulation or in potting compounds or conformal coatings are particularly hazardous because the boundary between a solid dielectric and a gas of lower dielectric constant gives rise to an increase in electrical stress on the gas. This often is enough to cause breakdown in the gas, which in turn results in heating and decomposition of adjacent insulation.

Grounded shields should be used over the insulation of high voltage cabling whenever possible to contain strong electric fields in the region immediately adjacent to the insulation and to reduce the likelihood of voltage breakdown in the volume outside the insulated wires.

Dunbar (Reference 12) shows that the voltage developed across the gas in a capacitor with a layer of gas and a layer of solid dielectric between the plates is given by

$$
V_{g}=\frac{V}{\frac{t_{1}}{t_{2}{ }^{\epsilon}}+1}
$$

where $V$ is the applied voltage, $t_{1}$ is the thickness of solid dielectric, $t_{2}$ is the thickness of the gas layer, and $\epsilon$ is the dielectric constant of the solid. The dielectric constant of the gas is assumed to be near unity. The quantity that 
determines gas breakdown is the electric field intensity. If we determine the electric field in the gas in a void and divide it by the field that would exist without any solid insulator, we find that the electric field in the gas in a void may be many times that which the gas would experience without the presence of the solid insulator.

Another phenomenon that must be guarded against in the case of insulated wires is a progressive type of failure known as "treeing." In this condition, a lesion in the insulation is produced by excessive electric field or by impact of charged particles from an electrical discharge in the gas near the insulation. Under continued electric stress, the lesion progresses as a series of branched channels through the insulating layer. The similarity of the appearance of the branch channels to the branches of a tree gives rise to the name. Some insulating materials (such as polyethylene, polypropylene, etc.) are especially susceptible to this type of failure. The only method of avoiding treeing failure is to keep the applied fields well below those which cause the failure by eliminating sharp corners and points. In some instances, the applied field will be less than the corona initiation level for the conductor without insulation. The dielectric strength and resistance to arcing for a few insulating materials are given in Table 2 (Reference 13).

The arc resistance is the time (seconds) that a material can endure an arc across its surface before electrical breakdown. This is not related to the ability of the material to endure electrical stress without treeing.

One further consideration with regard to insulation is that some materials are more likely than others to form a conducting path after a glow or arc-over has occurred. This is called "tracking." If such a path of reduced resistance is formed, then subsequent breakdowns occur more readily and lead to further insulation breakdown. The material least likely to form such conducting paths should be used.

b. Encapsulation. There are various ways of enclosing a high voltage system to prevent voltage breakdown from occurring; those most frequently used in space vehicles are pressurization in sealed containers, potting, and conformal coatings.

The rationale behind the pressurized container method is that since the environment of the high voltage system can be established as a safe environment free of breakdown troubles, then it can be maintained by a sealed container. Usually, a water-free gas is used at a pressure slightly greater than sea level atmospheric pressure. Several gases which have been popular 
Table 2

Dielectric Strength and Arc Resistance for Selected Insulation Materials Suitable for Molding, Extrusion or Casting.*

\begin{tabular}{|c|c|c|}
\hline Material & $\begin{array}{l}\text { Arc Resistance } \\
\text { (seconds) }\end{array}$ & $\begin{array}{c}\text { Dielectric } \\
\text { Strength } \\
\text { (volts per mil) }\end{array}$ \\
\hline Acetal resin copolymer & 240 & $500-2100$ \\
\hline Acrylic resins & no tracks & $400-500$ \\
\hline Acylonitrile Butadiene-Styrene & $70-80$ & $350-400$ \\
\hline Alkyd molding compound & $175-235$ & $300-350$ \\
\hline Cellulose acetate & $50-310$ & $230-265$ \\
\hline Cellulose acetate butyrate & unknown & $250-400$ \\
\hline Ethyl cellulose & $60-80$ & 800 \\
\hline Diallyl phthalates & $105-140$ & $350-400$ \\
\hline Epoxies & $120-300$ & $300-500$ \\
\hline $\begin{array}{l}\text { Fluorinated ethylene and propylene } \\
\text { (copolymer) }\end{array}$ & $>300$ & $500-600$ \\
\hline Mica-glass bonded & $240-300+$ & $350-400$ \\
\hline Neoprene & unknown & 300 \\
\hline Nylons with glass fibers & $0-150$ & $400-550$ \\
\hline Phenolic molding compound- unfilled & tracks & $300-400$ \\
\hline $\begin{array}{l}\text { Phenolic molding compound with glass } \\
\text { fibers }\end{array}$ & 0.4 to 150 & $140-370$ \\
\hline Phenylene oxide resins & unknown & 500 \\
\hline Phenylene oxide resins with glass fibers & 120 & 1020 \\
\hline Polychlor otrifluoroethylene & $>360$ & 530 \\
\hline Polyethylene, irradiated & unknown & 2500 \\
\hline Polyolefin & unknown & 1300 \\
\hline Polypropylene with glass fibers & 75 & $300-475$ \\
\hline
\end{tabular}

* These values are obtained under standard test conditions and may not be obtained in engineering applications. 
Table 2 (Cont' $d$ )

\begin{tabular}{|l|c|c|}
\hline Material & $\begin{array}{c}\text { Arc Resistance } \\
\text { (seconds) }\end{array}$ & $\begin{array}{c}\text { Dielectric } \\
\text { Strength } \\
\text { (volts per mil) }\end{array}$ \\
\hline Polystyrene (heat resistant) & $60-135$ & $400-600$ \\
Polytetrafluoroethylene & $>300$ & 480 \\
Polyvinyl chloride & unknown & $750-900$ \\
Polyvinylidene fluoride & $>50$ & 1300 \\
Silicone, mineral filled & 230 & 390 \\
Styrenes & $23-40$ & $50-425$ \\
Viton, fluoroelastomer & unknown & 350 \\
\hline
\end{tabular}

in sealed high voltage systems are listed in Table 3. Notice that the noble gases are not included. Experience with helium and argon has shown that, even at atmospheric pressure, they are not effective in preventing electrical breakdown. All the gases listed in Table 3, except hydrogen, are nonflammable and nonexplosive; most are nontoxic (Reference 13).

Pressurization has found favor in many sounding rocket programs, but has not been used extensively in orbital flights. The major problem with the longer orbital flights is in keeping the gas leakage of the container sufficiently small, especially in the space environment. One method is to provide a reservoir to replace gas lost by leakage.

The potting method is very commonly used in long term space flights. In potting, the parts of the system are firmly mounted, scrupulously cleaned, then covered with a potting material that polymerizes in place. The end result is a solid block of potting compound with the high voltage system completely encased. Polyurethane, silicone rubber, and epoxy resins are frequently used as potting compounds. Care must be taken to prevent stresses in the potting compound from producing mechanical damage to the circuit. Great pains must be taken to avoid entrapment of gas or vapor in the potting compound or between the potting compound and the high voltage system. The earlier reremarks about voids in insulation apply directly to voids in potting materials. 


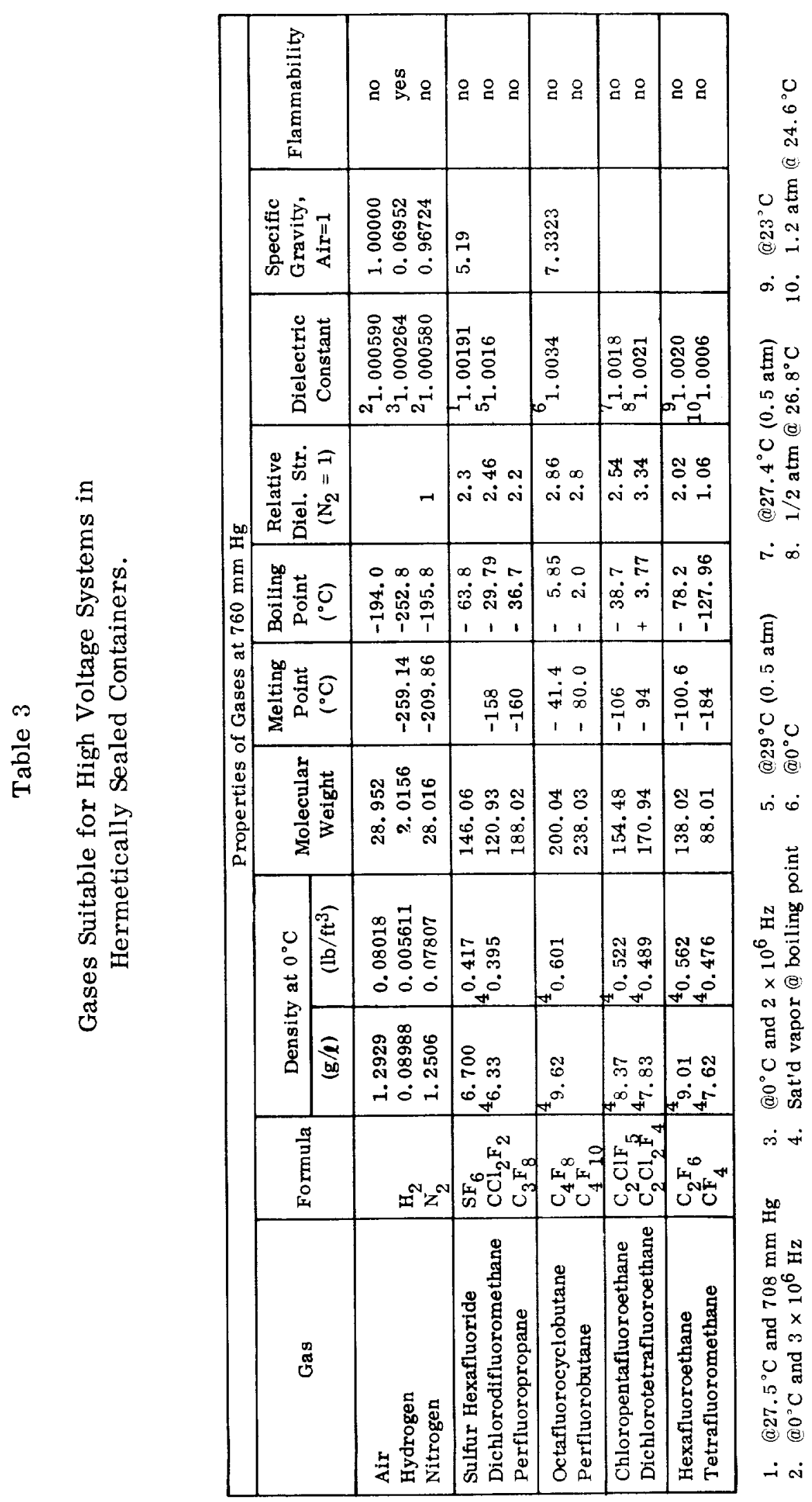


In conformal coating, the parts of the circuit which carry high voltages are laid out in a reasonably accessible array, very carefully cleaned and, if necessary, primed, then one or more layers of coating material are laid on by brushing, spraying, or dipping. Dipping is the favored method because it is most likely to produce a continuous coating without gas pockets. The coating is designed to have adequate dielectric strength to prevent breakdown from electrical puncturing. It also prevents short circuits caused by conducting debris that sometimes appears after launch. The parts are so spaced that the region outside the conformal coating is quickly evacuated when the system is placed in the low pressure of space. It should be noted that in many cases conformal coating is followed by potting to take advantage of the strong points of both methods. Both the potting and the conformal coating methods provide a degree of mechanical protection in addition to guarding against electrical breakdown. Epoxy resins are the most frequently used conformal coating materials.

c. Venting. A quite different approach to prevention of voltage breakdown problems is simply to open the system to the space environment so that gas in the high voltage system can readily escape into space. Hopefully then, the pressure near the high voltage components drops below $10^{-6}$ torr and breakdown is prevented by the high dielectric strength of hard vacuum $\left(\sim 5 \times 10^{5}\right.$ volts $/ \mathrm{cm}$-something like 20 times as great as that of dry air at sea level). This is called venting. It is an excellent method in principle, but, as is so often the case, great care must be exercised to assure that the principle is applicable to the real situation. For example, continuous evolution of gas from high vapor pressure materials in and around the high voltage system may prevent achievement of the desired low pressure environment. Or, gas evolved from the spacecraft from propulsion or control jets, from waste products, or absorbed gas layers may raise the pressure intolerably. Also, penetrating radiation or charged particles from space may make the volume near high voltage components sufficiently conductive that breakdown will readily occur.

It is almost always true that a high voltage system must be enclosed to some extent. It needs to be protected from dust and dirt and mechanical damage on the ground. In flight it is often necessary to shield the components from sunlight and charged particles from space. In order to apply the method of venting, some holes need to be located in the enclosure. The size and location of these holes must be related to the volume of the container and the gas load that may be expected to evolve from the high voltage system and its environs in such a way as to permit the pressure near the high voltage components to fall very rapidly when the pressure outside the enclosure is reduced. Two convenient rules of thumb have resulted from studies of vented enclosures: First, the pressure near the high voltage system should drop to 
approximately 37 percent of its initial value in one second when the pressure outside the enclosure is suddenly reduced to a very low value; and second, an alternative and roughly equivalent to the first, is that for every liter of volume in the enclosure there should be one square centimeter of area of evacuation port. Compliance with the first rule can be readily determined experimentally once a model is available. Compliance with the second rule can be determined by calculation, but considerable care must be taken to be sure that tortuous or narrow channels between parts of the high voltage equipment or between it and the walls of the enclosure do not invalidate the calculation.

Venting is a favored way of avoiding breakdown in high voltage connectors. High voltage cable connectors have given so much breakdown trouble that it has been the tendency to avoid their use. It has been found, however, that, where such connectors must be used, a substantial share of the voltage breakdown problems are avoided by drilling holes in the sides of the connectors to allow gas to escape readily.

\section{Ionizing Agents}

Since most gas discharge phenomena characteristic of electrical breakdown problems in spacecraft are triggered by the presence of one or a few charged particles near the high voltage leads, any steps that reduce the number of charged particles are likely to be helpful. Solar electromagnetic radiation in space will produce positive and negative charged particles by photoionization of gas molecules as well as electrons by photoemission from solid surfaces. This source of charged particles can be minimized by allowing sunlight to reach only those parts of the high voltage system which require it for successful operation; e.g., the detectórs in an experiment to measure solar radiation, or the essential elements of a sun-seeking guidance device. Energetic charged particles, like cosmic rays or solar wind particles, or even energetic trapped particles, may penetrate to the vicinity of high voltage systems and, either through their own presence or by secondary particles, trigger electrical breakdown events. The lower energy charged particles of solar plasmas, trapped radiation belts, and the ionosphere are also hazardous because, being much more numerous than the higher energy particles, they can significantly lower the breakdown voltage by accumulating in the vicinity of high voltage leads. The introduction of ion traps for particles up to a certain energy, and the shielding of high voltage elements by surrounding them with solid material are measures which have proved reasonably effective against electrical breakdown caused by charged particles. Higher energy particles will still penetrate, but since they are relatively few their adverse effects are often tolerable. Gamma rays and hard $\mathrm{X}$-rays will produce ionization that can be a hazard to high 
voltage systems, but these rays are so penetrating it is not usually feasible to shield against them. The best plan is to locate high voltage equipment as far from such radiation sources as possible.

One other way to avoid ionization effects is to avoid the presence of gases or vapors that readily produce ions; for example, water vapor is quite readily ionizable. Water absorbed on parts of the equipment or trapped in potting compounds may be released slowly in the space environment to cause trouble over a long period of time. Recommended procedures are to degas all components as much as possible prior to launch and to minimize exposure of the equipment to humid atmosphere between the time of degassing and the time of launch. Design features which facilitate this kind of handling should be incorporated.

\section{Accidental High Voltage}

Accidental application of high voltage to spacecraft ensembles has resulted in some equipment failures. Design features should be incorporated to reduce the danger of accidental high voltage. Static electricity has caused some trouble, especially in clean room operations where clothing of synthetic fibers is worn and where the humidity is kept quite low, the likelihood of "zapping" when one walks across the room and then touches a component is quite high. Circuits and containers of electrical equipment should be designed to minimize spark damage of delicate circuit elements.

Another type of accidental high voltage is caused by capacitors that are left charged from a previous operation. When a circuit including such a capacitor is taken through the critical pressure regime (Paschen curve's minimum voltage, see Figure 2), unexpected and damaging electrical discharges have occurred. The incorporation of suitable bleeder resistances is an obvious solution.

Another class of accidental high voltage is transient impulses. The subject is so broad that it cannot be treated in detail here; however, it is such a wellknown field that it is sufficient to remind the designer that transients can and do cause failures, and should be designed against.

Accidental application of high voltage while the equipment is subjected to the critical pressure region must also be avoided. Such accidents may occur when low and high voltages are linked and the operator does not consider the pressure effects when he wants to test the low voltage side. Accidents can also occur when the wrong signal is sent to the equipment under test, or when 
the equipment is working at high voltage and a failure occurs in the vacuum system thus allowing an unexpected pressure rise.

\section{Designing to Minimize Damage}

As an extra protection feature, it is useful to design electrical systems so that as little damage as possible will result even if a breakdown does occur. There are several precautionary measures that can be used. Easily damaged components such as certain elements of printed circuit boards and certain solid-state devices must be physically separated from the regions of high voltage. Not only does this protect against excessive current and charged particle impact damage, it also avoids transistor damage caused by high electric field intensities. Also, to the greatest extent that is feasible, "overdesign" is recommended. For example, using 10,000-volt insulation in a 2000-volt circuit is not unreasonable. In addition, insulation of a type that does not deteriorate rapidly or form conducting channels when exposed to electrical discharges should be employed.

Carefully designed current and voltage limitation should be a part of every high voltage system. One device is to make the circuit impedance so large that the voltage source employed cannot deliver more than a 50 percent excess current beyond the rated load even with a dead short. Another approach is to design so that voltage drops sharply whenever current rises above a certain value. It might be required, for example, that 10 percent excess current above rated load would drop the voltage by 50 percent. Fuses must be used with due regard for the consequences; e.g., the possibility that an experiment might cut off in flight because of some temporary malfunction having no long term adverse effects. On the other hand, the failure of one part of an experiment, or of one experiment out of thirty may be a desirable alternative to having one malfunction deactivate the whole package.

Fusing for cut-off when voltage breakdown becomes extreme or very prolonged seems necessary. The use of recycling solid-state fuses, or some other approach to being able to turn on and try again, may offer freedom from the finality of blown fuses. It is often desirable to provide alternative circuits to ground as protective devices if a voltage breakdown should occur; for example, a dropping resistor and diode circuit to ground will, if the voltage starts to build up, divert the excess current to ground. 


\section{Designing for Frequent Testing}

One of the most persistent and frequently recurring problems is the necessity of testing to see whether the system works properly at rated voltage. The word is: Test every component, every subassembly, and every circuit, then test the integrated package. The idea is that voltage breakdown can result from so many causes and in so many ways that the most painstaking and meticulous design and fabrication and quality control procedures can sometimes fail to spot the trouble. Hence, test it and see if it works. Testing can be greatly facilitated by providing design features that meet the testing needs. Circuit features should be included which make possible significant tests without damage to components. Electrical contacts should be made accessible for testing at the appropriate fabrication stages. 


\section{DESIGN AND FABRICATION PRACTICES}

This part contains descriptions of various techniques, procedures, and materials that are being successfully used to build high voltage space systems. Three specific systems currently in use are described along with the methods used in these systems to avoid high-voltage troubles.

\section{Three Examples}

\section{A Cockroft-Walton Power Supply and a Multiplier Phototube}

In this system the power supply provides a series of voltages up to 1500 volts de to the dynodes of a photomultiplier tube. All parts of the system carrying voltages greater than 100 volts are enclosed in potting compound or insulated wires.

The phototube is operated at up to 1500 volts de with the cathode grounded. This introduces some complications in handling the phototube output signal since it is near the anode potential of +1500 volts. However, the electrical breakdown problems are much easier to solve with the cathode grounded, because the cathode is usually large in area and exposed. If it is at a high (negative) potential, the entire potential drop to grounded structure occurs within a short distance from the phototube envelope. The anode, on the other hand, is usually small and hidden well inside the tube. The ease of managing the breakdown problems is more important than the problems of signal handling. The tube has ten dynodes held at various potentials between ground and +1500 volts, as is usual.

The power supply is a Cockroft-Walton design, which is a sequence of series and parallel capacitors and diodes which take an ac input, rectify it and increase the voltage in steps as it progresses through the sequence of capacitors and diodes in such a way that the potential of each capacitor is determined by adding an increment to the potential of the preceding one. In the case of a 1500 -volt system with 10 steps (to supply the 10 dynodes) each increment is approximately 150 volts. The high voltage side of each capacitor in the chain is connected directly to the appropriate dynode of the phototube. Thus there are no bleeder or dropping resistors in the circuit. In the system being described here the input to the power supply is about 100 volts at a frequency of 300 to $500 \mathrm{kHz}$. This is brought to the power supply by shielded cable. The CockroftWalton system is laid out in a circular array and the entire assembly is potted into a pillbox-shaped container about 2 inches in diameter and 1 inch high with a $1 / 2$-inch hole through the center along the axis of the cylinder. The high voltage system is completely enclosed except for the contact points, which are 
left open on the bottom side, and the input power leads. The pillbox containing the potted-in power supply is obtained complete from the manufacturer, Matrix Research and Development Corporation.

On receipt, the power supply undergoes incoming inspection, and is then tested to determine satisfactory performance and delivery of the required voltages. Then short insulated leads rated at 5000 volts are fed through the center hole and soldered to the various connection points on the bottom side of the pillbox. Great care is exercised to see that good solder joints are achieved and that no sharp or jagged edges are left (see Figure 10). The entire unit is thoroughly cleaned again and then the whole bottom surface is potted with RTV60 in such a way that all soldered joints and the first $1 / 4$ inch of the insulated leads are covered with about a $1 / 8$-inch thickness of potting compound.* During this operation the leads are kept carefully separated so that no narrow space between two leads can trap gas or fail to receive potting compound. Also the leads are, as they pass up through the center hole, unpotted to maintain flexibility. The insulation on the wires is relied upon to prevent breakdown in the central hole. The leads are then clipped, so that they extend about half an inch above the power supply, and soldered to a circuit board.

Figure 10-Sharp edges and points

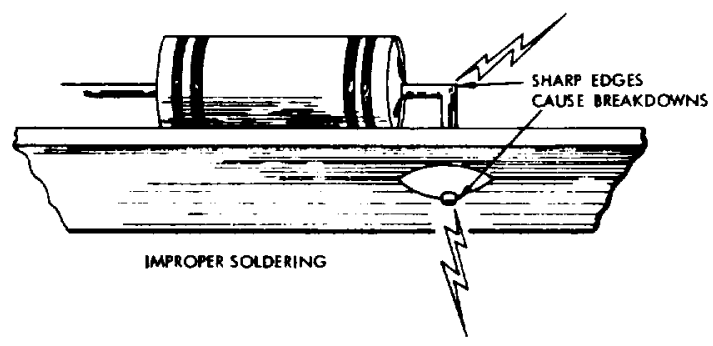
must be avoided.

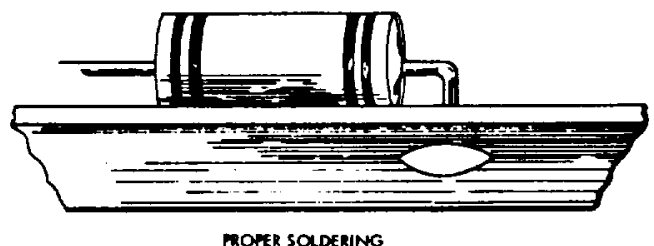

*Mr. E. R. Bunker of JPL advises us that RTV compounds are avoided as much as possible in JPL spacecraft because of outgassing problems and difficulty of eliminating voids. 
The circuit board is now ready to be connected to the phototube; but first the phototube and the way in which it is prepared to receive the voltage leads should be considered. The phototube, like all other parts, is subjected to incoming inspection and whatever tests may be required to determine compliance with specifications. The socket pins are unsoldered and the plastic tube base and attached pins are removed from the tube. (In many cases tubes are supplied without a base.) The tube is then carefully washed with solvent to remove all traces of dust, dirt, grease, fingerprints, and moisture, and is placed in an aluminum can. The can has an inside diameter about 1/4-inch larger than the outside diameter of the phototube so that potting compound can flow along the sides of the tube and provide both mechanical (vibration) and electrical isolation of the tube from the can. The can is about 1-inch longer than the phototube. The circuit board, which is small enough to fit inside the can, is now soldered to the tube leads. The assembly is ready for potting. The assembly, at this stage, is shown in Figure 11.

The potting compound used is the red variety of RTV-60. It is thinned with about 20 percent (by volume) of the recommended thinner, to reduce the viscosity so all spaces will be filled. The thinning also causes more flexibility in the end product, a desirable feature. RTV-60 is added until the circuit board and any components on it are covered. The assembly is placed in an evacuable chamber and the pressure is lowered until all air trapped in and around the potting material has been pumped out. This step requires some skill and judgment. The trapped air will make bubbles as it escapes from the potting compound. If there is much trapped air or if the surrounding pressure is reduced very rapidly, violent agitation of the RTV-60 can occur with formation of froth or with spattering of the material in unwanted places because of
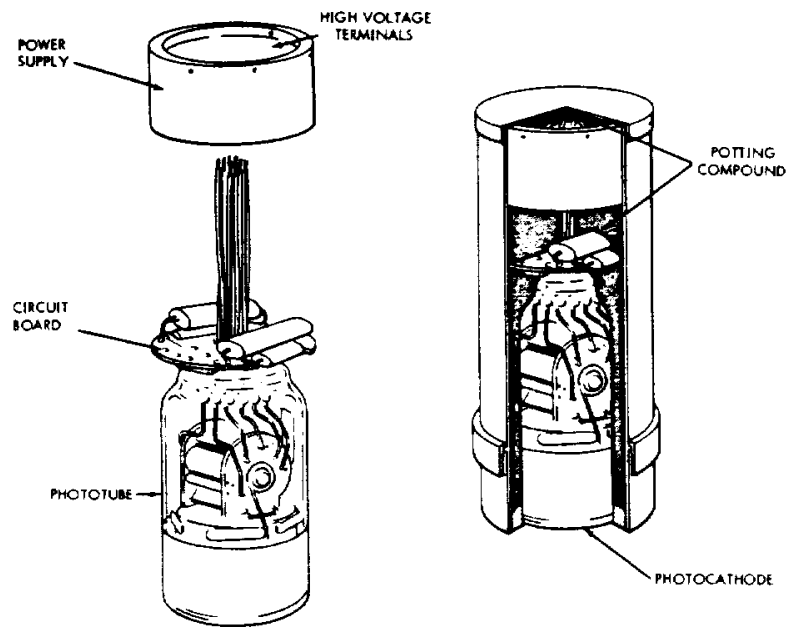

Figure 11 -Cockroft - Walton generator and multiplier phototube. 
breaking bubbles. When the bubbling stops all trapped air is gone. Normally this takes not more than a couple of hours. However, close inspection may show occasional small bubbles evolving for days. But it is not advisable to pump for very long times because volatile constituents of the thinned potting compound will be lost and the properties of the end-product will be different from what is desired. Thus the inexperienced operator is faced with a dilemma since too little pumping is bad and too much pumping is bad; but the decision as to just how long to pump is difficult to make. The best way out is to make the first few units under the instruction of an experienced operator. If this is not possible, experimental pottings should be made and tested for performance and sectioned to see whether any gas pockets were left. In this way the proper amount of pumping for the specific circumstances of a given fabrication may be determined.

After the trapped gas has been removed from the assembly, ambient laboratory pressure is returned and the potting compound is cured in the temperature-time regimen recommended by the manufacturer (with due consideration for the effect of the curing temperature cycle on the components of the system being potted). It is also necessary to consider the effects on other parts of the system of the vapors given off by the potting compounds during pumping and curing. These may produce undesi rable coatings or contamination on optical parts or detectors.

This procedure results in a system in which all parts carrying high voltage are completely encased in potting compound, except for the wire bundle connecting the Cockroft-Walton power supply to the circuit board; these wires are insulated with ratings three times greater than the maximum voltage to be applied, they are very short, and they are well vented so that no gas can be trapped by them during flight. The experiments for which this technique was developed are not required to operate in the "wormy" region (a picturesque term for the pressure range in which breakdown is most likely; first heard from W. E. Six, University of Chicago). However, they have operated and will operate through this region without failure and have compiled many years of successful orbital flight experience. The only breakdown failures which continue to interfere with the experimental studies are occasional brief discharges that are difficult to distinguish from real particle counts. These are sufficiently few that they constitute no great difficulty in most cases. It is worthy of note that simply changing the ground from cathode to anode results in an enormous increase in the number of false counts. A sketch of a completed unit is shown in the right of Figure 11. 
The voltage involved in this next system ranges up to 3000 volts and is direct current from a power supply that may serve one or several units such as phototubes or particle detectors. The power supply and the components closely associated with it, such as filter capacitors and current limiting resistors, are placed in a metal box that is filled with potting compound. Connection between the high voltage terminals and the leads required to distribute the voltage to the use sites is made by means of a glass-to-metal feed-through in the wall of the box. Shielded insulated cables are used as leads to the point of application of the high voltage. The leads a re kept as short and straight as reasonably possible (see Figure 12).

The procedure for potting the power supply is as follows. The power supply is designed to fit into the allotted space with at least $1 / 8$-inch clearance on all sides. A fused glass and metal feed-through with a Kovar sealing ring is installed in the power supply housing. The power supply in its container is placed in an evacuable chamber and pumped down until no more gas escapes from the potting material and until all gas that may have been trapped in the power supply is gone. (The chamber pressure need be no lower than 0.1 torr for this operation.) Then the potting material is poured, without breaking vacuum, into the power supply container to fill it right to the top. The top plate of the box is put on and screwed into place. When the potting material cures, it fills all the space in the container not occupied by circuit
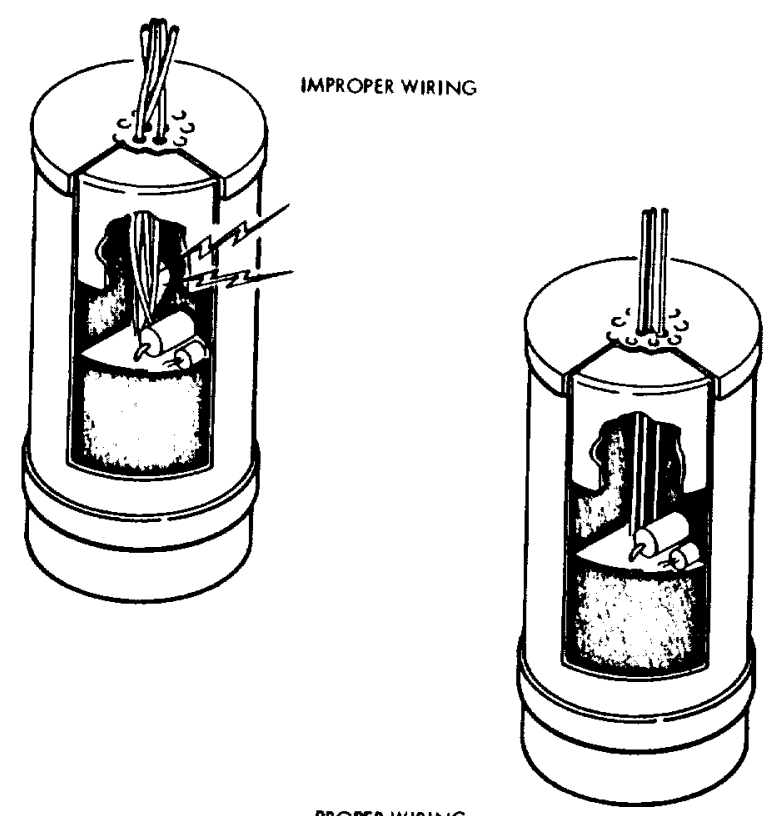

Figure 12 - Spaced parallel wires avoid gas pockets in potting. 
elements, and adheres to the top plate of the container as well as to the side walls and the bottom. Voids in the potting cause trouble as indicated in Figure 13. The potting compound used is Emerson \& Cumings $1090 \mathrm{SI}$, which is a low viscosity black material filled with tiny hollow glass balls. * This material is mechanically compatible with the aluminum housing and the electronic components. No stress breakage occurs due to temperature change, even if the equipment is immersed in liquid nitrogen. The potting compound with filler seems to have enough give that differences in expansion coefficient don't result in breakage. The filler also has a desirable electrical effect: it forces an electrical discharge to follow a tortuous path to get by the filler material and thus increases the ability of the potting material to restrain electrical discharges.

The curing temperature used for these assemblies is $50^{\circ} \mathrm{C}$. It is best to have the electronic assembly and the potting compound at $20^{\circ} \mathrm{C}$ to $25^{\circ} \mathrm{C}$

Figure 13-Voids in potting compound cause trouble.

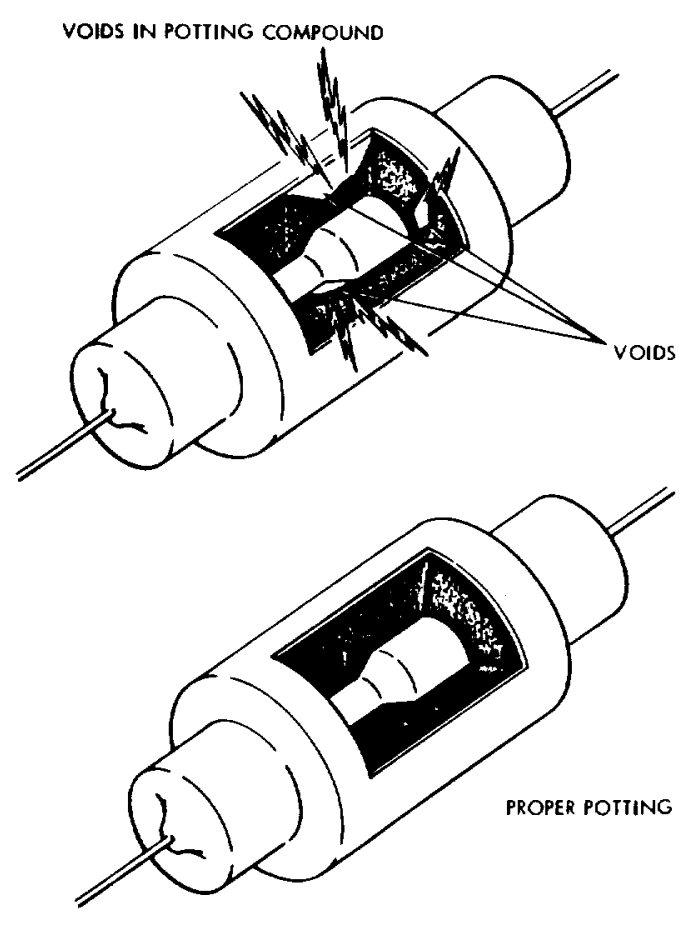

*Mr. E. R. Bunker, Jr., of JPL reports that JPL experience with 1090 SI with glass ball filling along with the use of gas escape paths in solid potting varies in some important respects from the results reported in this section. 
during pouring. Some attempts have been made to pour when the equipment is hot enough to drive off some of the adsorbed gas layers, but this has not worked well; gas pockets tend to be formed between the solid parts and the potting compound.

When the depth of the potting compound is great, for example, a 2-inch well, metal rods are placed in the potting compound during curing and removed afterward. The hole left by the rod provides an outgassing channel so that gas can quickly escape from the potting compound and from any open volume at either end. Typically four rods, each $1 / 6$ inch in diameter, are used to provide venting for a cylindrical section of potting compound having a 2-inch o.d., a 1-3/4-inch i.d., and a 2-inch length.

Transformer cores are potted in RTV 850 before they are inserted in the assembly for potting in E-C 1090 SI. This provides additional mechanical cushioning and also prevents undesirable penetration of the core by the $\mathrm{E}-\mathrm{C}$ 1090 SI.

The use of a glass header to make the connection to the high voltage power supply contained in the potting compound is important. Wires emerging from a potted assembly are assiduously avoided because of the possibility of gas being trapped near the wire or leaking through the wire insulation interface.

The wires used for distributing the high voltage use wrapped insulation 8 - to 13 -mils thick at a rating of 1000 volts per mil for a 2000 -volt supply. These are equipped with a grounded shield, open for the passage of gas at both ends. The end of one line is soldered to the lead in the header, the other is soldered to a board. Distribution connections are soldered to the circuit board and to the application points. All soldered joints are then conformal coated. No connectors are used. No wires are permitted to enter or emerge from potting. Potting boots are avoided.

The principal precautions taken with this unit to avoid damage due to breakdown are:

1. Provide escape routes to ground for accumulated charge.

2. Test the system through the wormy region with voltage on.

3. Test new power supplies on separate low voltage busses.

Escape routes are provided by connecting vulnerable points to ground through a resistor and a diode. When charge starts to build to dangerous levels because of an electrical breakdown, the charge is allowed to leak off to ground. 
The test of the system in the critical pressure range, with the voltage on, is a survival test. A circuit must be able to endure any discharges that occur under these conditions without suffering any permanent impairment of function. Testing of all new circuits on separate low voltage busses protects the other circuits from damage that might result from a gross failure of the new circuit when all share a common bus. Once the new circuit has been demonstrated to perform properly it can safely be placed on a common bus with other tested circuits.

Turn-on transients are reduced to a tolerable level by a programmed switch-on circuit that turns on the current in steps, bringing it up to the required value in about one second.

\section{Voltages to 15 Kilovolts}

The system in this example provides and uses voltages of 2500 volts, 3600 volts and 15,000 volts. There have been no in-flight problems recognizable as electrical breakdown phenomena. This is a Cockroft-Walton generator system with voltages picked off at appropriate stages as already described. The practices followed to insure success are as follows.

The system is either left open with unshielded wires and soldered joints and is carefully vented to insure rapid removal of gas or vapors, or it is potted solid. The open or unpotted procedure is a simple and effective approach to avoiding breakdown. As in the case of enclosed systems, sharp points and edges are to be avoided. The parts of the high voltage system must be scrupulously clean so that they are free of any deposits that will evolve appreciable amounts of gas or vapor. Also there must be no debris either on the high voltage parts or in the vicinity that can possibly lodge between parts at different potentials and result in a conducting path. When such precautions are taken and the volume containing high voltages attains pressure less than $10^{-5}$ torr, trouble-free operation is assured.

The speed with which a vented system achieves sufficiently low pressures for safe operation depends on the configuration of parts, on the rate at which gas is evolved by various mechanisms such as release of adsorbed surface layers, evaporation and sublimation, and on the size and location of the openings through which gas can escape to the external low pressure region. An analytical approach to the determination of the rate of pressure decrease has been developed (Reference 14). This analysis, which is organized for computer calculation, is a very useful guide to the design of vented systems. It must, however, be supplemented by empirical determinations of the rate at 
which the pressure drops because there are many factors determining the outgassing rate that cannot be known sufficiently well to provide the needed inputs to the calculations.

If potting is the method used, a transparent silastic potting compound is used whenever possible and the potted assembly is inspected visually with a low power microscope. This permits one to see voids, gas pockets and places where the potting compound has failed to adhere to the components. When it is necessary to keep out visible light, as, for example, with a phototube, an opaque variety of silastic has been used successfully. In that case extensive electric testing has to take the place of visual inspection. A primer is always used on every component to improve adhesion between the potting compound and the hardware. The primer is always applied by dipping rather than brushing. (See Figure 14.) Gas escape paths are provided so that trapped gas pockets will not form. After pouring the potting compound to fill the cavity containing the high voltage system, the surrounding pressure is reduced by pumping, which is continued until no more gas bubbles can be seen escaping from the surface.

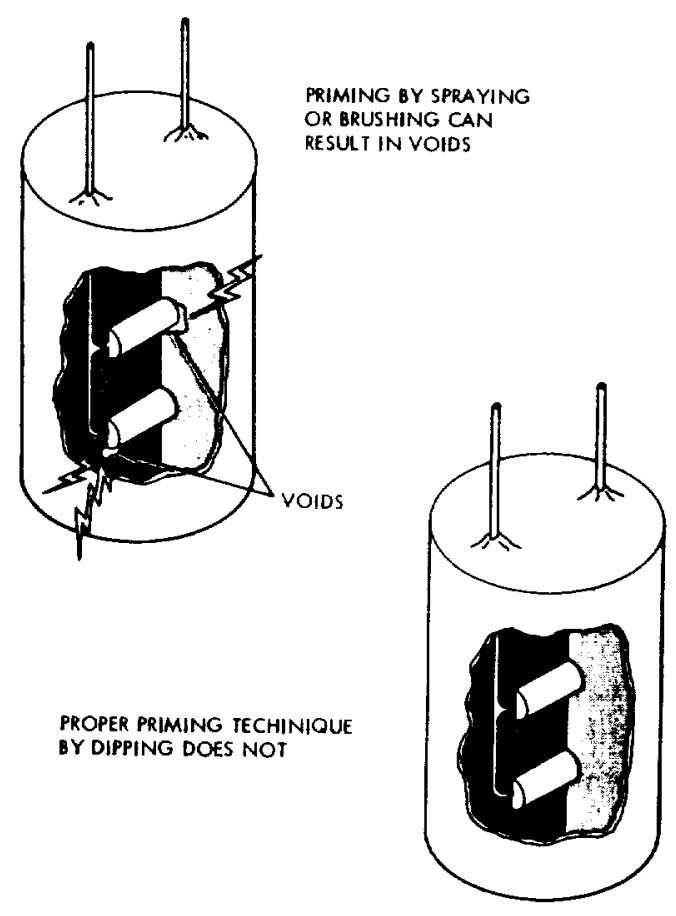

Figure 14-...Proper priming results in good adhesion between hardware and potting compound. 
Absolutely no connectors are used. All junction points are soldered with the exception of the 15-kilovolt system described below where a simple mechanical contact suffices. Whenever possible the high voltage is developed at the point where it is to be applied so that there is no need for high voltage leads. This is illustrated schematically in Figure 15. The unit requiring 15 kilovolts rests directly on the rounded central stub where the voltage is developed. In cases where distribution of high voltage is unavoidable, special cabling is used. Teflon-insulated wire with two added teflon sleeves is used.*

In the construction of this 15-kilovolt system, fabrication personnel are not relied upon to know what steps to take or to remember to take every precaution. The ultimate user of the equipment undertakes to instruct the fabricator, whether he be an in-house technician or a vendor, in the detailed steps to be followed. The user then follows up with frequent over-theshoulder looks at the fabrication process.

Extensive testing is done, including a period of from a few days to a few weeks in hard vacuum. When a failure occurs during test, a very detailed failure analysis is done so that it is known exactly what part failed and, to the greatest extent possible, the mode of failure and the conditions that existed just before, during and after failure.

Figure 15-Illustration of direct contact 15 kilovolt assembly.

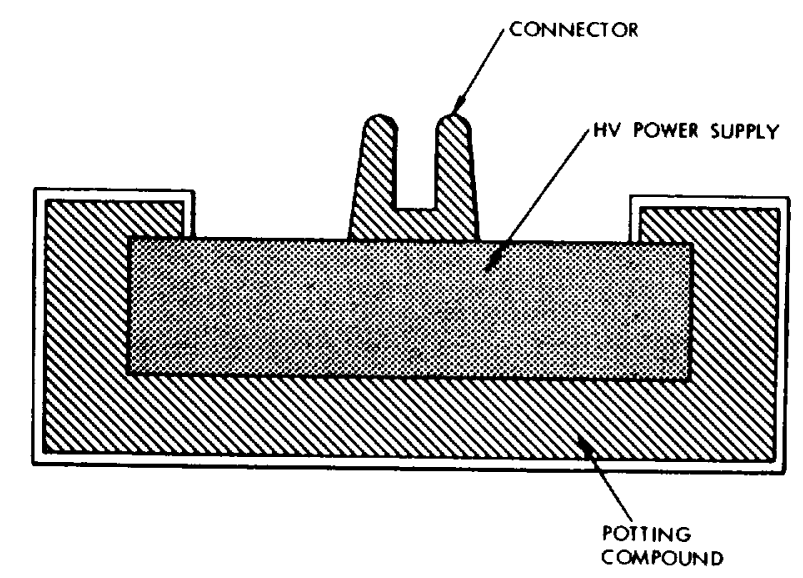

*Such a procedure is very vulnerable to trapped gas between the sleeves and is not usually recommended. It is described here because it is in fact the method being used. 
Pressure gauges are used in flight equipment, and turn-on is delayed in flight until the gauge reading gives a good margin of safety. This normally requires a few days in orbit before turning on.

\section{High Frequency Practices}

The material included so far in this section has been related primarily to dc problems. Although some of the systems described used chopped or timevarying voltages, the voltages were unidirectional and the time variation was at frequencies not greater than a few kilohertz; the electrical breakdown behavior of systems is about the same for dc systems and ac systems up to such frequencies. However, at frequencies greater than $10^{6}$ hertz, new phenomena associated with charged particle resonances begin to be important in breakdown behavior.

The effect of multipacting on the Paschen curve at low pressures is illustrated in Figure 16 (Reference 10). At frequencies in the 20 to $2000 \mathrm{MHz}$ range, where most of the communications between spacecraft and ground stations takes place, design and fabrication practices somewhat different from those for de have evolved.

Since of necessity an antenna must be exposed, there is no possibility of entirely isolating the high voltage. The only way to prevent electrical breakdown in the space around the antenna is to keep the voltage below the breakdown level. In the critical pressure region this requirement means that voltages cannot be allowed to get larger than about 150 volts. By careful design, flight communication systems are built in which 10 watts may be radiated without having voltages exceed 100 volts.

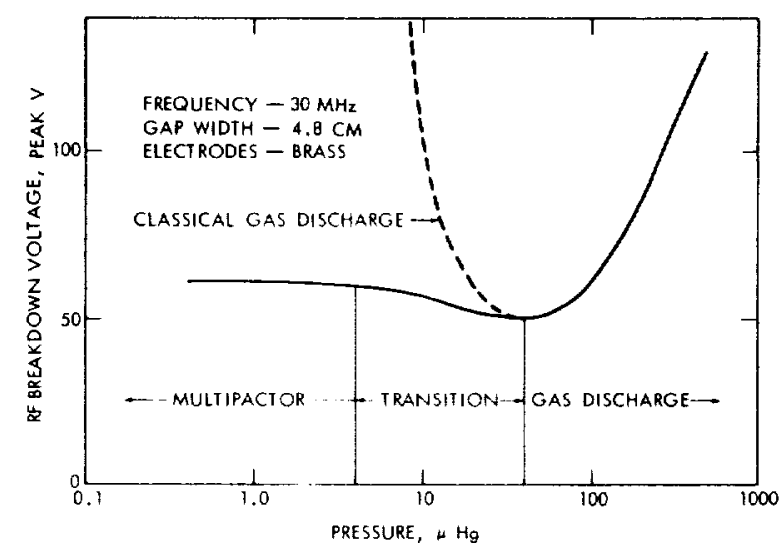

Figure 16-Paschen's Curve as modified by multipacting. 
The RF circuits themselves may be protected from breakdown either by encasing them in hermetically sealed containers or by encapsulation in foam. A sealed container with a suitable insulating gas (see Table 3) at a pressure a little greater than sea level ambient pressure is a very effective way to prevent breakdown. This technique is used frequently in sounding rocket flights. The problems of achieving and maintaining a sufficiently low leak rate to insure satisfactory performance in space for flights of a year or more duration seem so great that few such flights have used hermetically sealed containers.

The more common practice is to fill the circuit compartment with a foamin-place material like Eccofoam; however, the foam will result in a need for complete retuning of the circuit. This restricts the motion of charged particles and effectively triples the voltage required for breakdown to occur.

One other device that has seen some application is to place the RF components in a clean, empty box with the lowest possible yield of secondary electrons due to charged particle impact. All plastics and organic materials are carefully avoided and, as completely as possible, the environment of the circuit consists of glass and metal. The conainer is well vented so that the internal pressure falls rapidly in space. This approach is less effective than foaming. It provides no protection in the critical pressure region.

$\mathrm{RF}$ breakdown phenomena are usually not as damaging either to the RF equipment or to other nearby circuits as are the dc type breakdowns.

Details of a procedure for avoiding breakdown failures in a Gemini transponder are given by Brock and Vester (Reference 10). The transmitter had a peak output ranging from 1150 to 1400 watts. The high voltage on the transmitter was 1650 volts. The steps taken to avoid damage from breakdown were as follows. Power supplies were made short circuit proof by limiting current so that no components were loaded beyond their rating even under short circuit conditions; furthermore, the design was such that a direct short on the high voltage side would not give fault currents large enough to cause lowpower semiconductor components to fail. Equipment was designed to ope rate satisfactorily in the critical pressure region even though it was not planned to use it in that region. RF components were not sealed and vent holes were added. The adequacy of the venting was determined by testing how long it took the glow discharge to disappear when pumped down with RF power on. Where feasible, twice the rated power was used for the tests. 


\section{Conformal Coating Procedures}

An interesting and valuable example of the procedures followed by laboratories most consistently successful in avoiding electrical breakdown failures is offered by the following specification prepared and used by the Laboratory for Astrophysics and Space Research of the University of Chicago. One should note the detail with which the procedure needs to be described to insure that the persons using this outline are informed of all the precautions that must be taken.

\section{CONFORMAL COATING PROCEDURES}

\section{PREPARATION}

1. Inspect module, board, stick, etc. to insure all condensers, resistors, wires, etc. are firmly supported or touching a firm supporting structure in order that conformal coating will secure them firmly into place.

2. Be certain that inspection code or marks are on material to be conformal coated.

\section{PROCEDURE}

1. Wash the unit in a fresh bath of isopropanol alcohol. A used or unused tray should be used to wash the unit, with positive assurance that the tray is not contaminated with any other foreign materials of any kind.

2. The unit should be washed for no less than one hour and no more than two hours.

3. The unit should be agitated frequently in order to flush away dislodged particles. In some cases, the use of a small brush may be necessary to assure proper cleaning of spots of contaminated areas. In all cases, the brush should be one which has not been used for any other type of cleaning. 
4. Remove the unit from the wash bath. Allow it to drain for five minutes. It should not be allowed to rest on benches or any other areas unless clean, non-waxed, non-oily paper such as Scotch 590 is used. If a container is used, the history of the container should be such that it shows no period of contamination.

5. The unit should not be touched with the hands from the time that the unit is washed until the coating is polymerized unless clean finger cots or other similar protection are used. Handling can also be accomplished by using clean tweezers or similar suitable devices.

6. The unit is placed in a vacuum chamber with pressure reduced to $100 \mathrm{microns}$ or less after it has drained for the proper period.

7. No backstreaming oil from the forepump or diffusion pump shall be allowed to enter the chamber into which the unit is placed. This can be accomplished by filling a dry ice trap at least one half hour before the unit is placed into the vacuum chamber. Periodic checks of chamber cleanliness using glass sides to detect backstreaming shall be done.

8. After the unit is in the vacuum chamber, elevate the temperature to $57^{\circ} \pm 3^{\circ} \mathrm{C}$ and maintain the pressure and temperature for a minimum of two hours.

9. Remove the unit and mask the terminals which should remain free of the coating or apply mold release. When applying mold-release products, extreme care should be taken to see that no small particles of mold release contaminate any part of the area in which the unit is to be placed. If it is suspected that the unit is contaminated, it is necessary to repeat the cleaning procedure. 
Note:

1. The unit or units should not be left to remain in the vacuum chamber without the trap filled.

2. The unit or units should not be removed from the vacuum chamber until it is ready to be coated. They should not be left to stand a round out of the chamber once they are cleaned. If it is necessary to have the unit out of the vacuum chamber for a short period of time before the coating is applied, it should be stored in a clean container within a clean wrapper to prevent it from being contaminated.

\section{MIXING AND COATING}

1. Proper mixing proportions are:
a. Bonding agent
100 parts by weight
b. Calcofluor White LVZ
0.02 parts by weight
c. Hardener B
6 parts by weight

2. Select the proper mixing container which should be a non-contaminated container, preferably of the polyethylene type. In addition, a container for the unit of such a size as to allow the unit to be covered with the mixed epoxy and a minimum volume expansion of 15 to 20 times the epoxy during the outgasing should be selected.

3. Weight out the proper amount of bonding agent. (At no time should less than $10 \mathrm{gms}$ be mixed at one time.)

4. Weigh and add the proper amount of calcofluor white LVZ conc. Mix mechanically for five minutes.

5. Add the proper amount of weighed hardener $B$ and mechanically mix for five minutes. 
6. Check humidity in preparation room. If above $70 \%$ relative, do not coat but proceed to reduce humidity to a safe value.

7. Saturate the unit with the epoxy by pouring it over the unit in the container. Insert the unit in a vacuum chamber and allow it to outgas. Outgasing will be complete when very little bubbles continue to come out of the epoxy. This will take approximately from ten to fifteen minutes.

8. Upon the completion of outgasing, remove the unit from the vacuum chamber and inspect it to be certain that complete coverage has been attained. Remove the unit from the epoxy and mount it, hanging it in such a manner to allow the excess epoxy to drip from one corner. (Mounting can be from a corner mounting bracket, a solder lug which is to remain clean, etc.) Allow the unit to hang draining until epoxy starts to polymerize.

Polymerization can be determined by noting when the drops become harder to leave the unit and become stringy. The length of time for this to occur will depend upon the time elapsed until this phase of the coating occurs. Normal pot life is about 45 minutes which would leave from about 20 to 30 minutes for stringiness to develop.

The unit is precured by mechanically rotating it in front of infrared lights until polymerization has set in to the point where the epoxy does not flow. Limit temperature by suitable spacing between lamps and work to $57^{\circ} \pm 3^{\circ} \mathrm{C}$.

The unit is put into an oven and post-cured at a temperature of $80^{\circ} \mathrm{C}$ for two hours. After the post cure, it is allowed to reach room temperature and is washed again in fresh isopropanol alcohol for five minutes. The unit is 
allowed to dry and is ready for final as sembly upon completion of the wash in isopropanol alcohol.

Note:

If the unit is put directly into the oven and rotated at $80^{\circ} \mathrm{C}$, the pre-cure cycle is not necessary, but the post cure time should be three and one half hours.

\section{Connectors}

Some designers of high voltage flight equipment avoid the use of electrical connectors entirely, relying solely on soldered joints. On the other hand, some equally successful designers do use connectors. The key to successful use of connectors is to be certain that no gas can be trapped in the connector body in such a way as to leak out slowly and result in a critical pressure environment for the high voltage leads in the connector long after one would expect all gas to have diffused into space. In order to accomplish this, connectors are vented by drilling holes through the body and insulation to permit ready egress of any gas. Figure 17 shows an adequately vented connector.

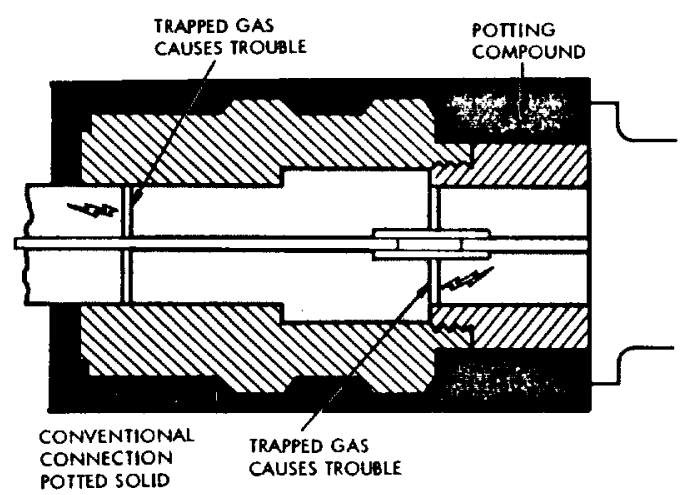

Figure 17 - Vented electrical connectors avoid trapped gas.

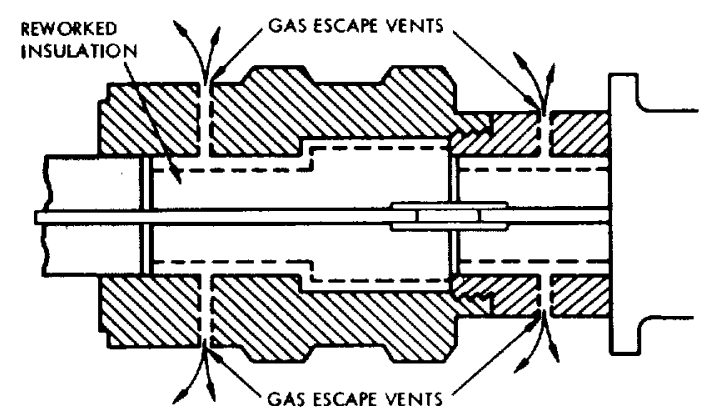


This connector passed all environmental tests and gave no trouble in flight. Figure 17 also shows a failed connector. This was a commercial connector which was simply encased in potting compound after the connection was made. This served to make a nearly gas tight seal around the electrical leads. During vacuum testing the gas slowly leaked out. When the pressure around the leads got low enough, a breakdown occurred which drew large currents and destroyed the connector.

\section{Recommendations from JPL}

The following statement of recommended practices was supplied to us by Mr. E. R. Bunker, Jr., of JPL. They are not intended to be a complete set of safeguards against breakdown problems; however, they differ in some ways from other practices described in this section. Many are included here to provide additional guidance.

1. In regions of high voltage gradients in high voltage equipment, only materials which can achieve an interface-free bond within encapsulation compounds should be used. This would eliminate the use of Kel-F, Nylon, Dacron, PVF, Kynar, Mylar, polyethylene and silicone polymers.

2. Insulated wire used in high voltage systems should be screened 100 percent by applying twice rated voltage plus 1000 volts for one minute so that any possible voids or weak places in the insulation may be detected. A corona detection network should be used. If the high voltage wire is shielded with an insulating sleeve shrunk on the shielding, the only way voids may be detected in the insulation is to place the wire in a chamber at the critical air pressure region with provision for allowing air trapped between the shielding and the insulation to leak out and then to apply the test voltage.

3. The application of Stycast $1090 / 11$ should be controlled by an appropriate process document such as JPL ZBE-1037-0002 for encapsulation of electronic modules.

4. To minimize thermal expansion effects, the volume of potting compound to the total volume of the module ideally should not exceed 15 percent.

5. Corona detection tests should be performed on every encapsulated high voltage module.

6. In places where high voltage terminals must have an encapsulation or a coating over them to eliminate corona or arcing, always prime the terminals first with an epoxy polyamide, then coat the primed areas with polyurethane. 
7. Always encapsulate with vacuum equipment in order to remove entrapped air.

\section{Approved Practices}

The following is a list of one-sentence reminders of the best practices to follow (Reference 15).* Most of them are included in one form or another in the main text of this section. It is not possible to arrange the reminders in order of importance because their relative importance is different in different situations.

1. Select potting material compatible with the job.

2. Prepare the surfaces for potting by careful cleaning, by removal of all debris and by priming.

3. Use materials that are fresh and not outdated.

4. Follow the manufacturer's instructions for measuring, mixing, pouring and curing.

5. De-aerate to insure corona protection.

6. Protect components from hard epoxies with elastic coating or potting compounds.

7. Avoid thick potting sections which will crack and craze.

8. Check potting to be sure it adheres to surfaces.

9. Avoid polysulfide potting compounds and polyvinyl chloride formulation because of outgassing.

10. Avoid those additives to polymeric materials which will adversely affect their outgassing characteristics.

*Dorrell, R. and Cooke, P., "Tips to Experimenters," available through NASA, Goddard Space Flight Center, December 1966. 
11. Because different cans of the same batch of potting material behave differently, make a test curing every time.

12. Delay turn on of high voltage until certain the pressure is low enough.

13. Vent all connectors.

14. Design for continuous operation through the critical pressure region even though the mission may not require it.

15. In conformal coating use at least $1 / 10$-inch thickness of coating material.

16. Use components rated for at least three times the actual voltage you expect to use.

17. Avoid insulation of the sort that may trap gas.

18. Pot solid and inspect thoroughly.

19. If hermetic seals are used require that they maintain a leak rate less than $10^{-8} \mathrm{cc}$-atm $/ \mathrm{sec}$ throughout temperature and pressure cycling.

20. When venting be sure the vents are adequate by testing.

21. Take great pains to clean the equipment and keep it clean.

22. Spread the high voltage parts to keep voltage gradients low.

23. Avoid sharp points and corners both to keep the electric fields down and because conformal coating does not coat them well.

24. Use nonconductors instead of metal for structural parts where appropriate.

25. Keep dielectric discontinuities as small as possible.

26. For pressurized containers of high voltage equipment, gasses like sulfur hexafluoride and Freon should be used.

27. Before coating or potting be sure components have adequate mechanical support.

28. Do not apply voltage until curing is complete. 


\section{TEST CONSIDERATIONS}

A universal recommendation of successful high voltage users is that all high voltage systems should be extensiveiy tested. Two specific tests are usually recommended: survival of operation at sustained critical pressure; and hard vacuum (10-7 torr) test of sufficient duration to insure that any small words within an encapsulated circuit reach critical pressure. Some specific tests are spelled out in section V in the JPL specification that follows this section. See paragraphs 4.4.1.1 through 4.4.3.4 of that document.

\section{Test Opportunities}

The following sequence for testing should be given consideration during the design and development of circuitry for space use:

1. Thoroughly test the functional capabilities of the circuitry at the breadboard stage, both in air and hard vacuum. At this stage of development design defects are more easily found and corrected.

2. Production circuitry should be tested as extensively as possible before potting.

3. All malfunctions and fixes related to high voltage circuits should be recorded and carefully analyzed. This information will facilitate design reviews and help develop optimum tests.

4. Special consideration should be given to installing corona and breakdown detection capability in prototype equipment. This may only consist of isolating the various grounds (power, signal, and chassis) so that voltage and current wave forms and current levels can be examined, or it may require that appropriate test points be made readily accessible for detection circuits, or it may even require the incorporation of special detectors in the assembly.

5. If the potted unit is highly critical, conside ration should be given to having the unit $\mathrm{X}$-rayed in order to discover voids in the potting materials. Transparent potting materials and examination with a microscope will also suffice.

6. The need for adequate venting cannot be overemphasized. The venting should be sufficient to evacuate unsealed packages and outgassed material as rapidly as possible. The pumping capacity of the test chamber must be great enough that it does not limit the rate of removal of gas. With sealed 
units the most critical problem is the leak rate. Is it low enough so the container interior will not reach critical pressure during the design lifetime of the experiment or spacecraft?

7. If a circuit or sensor is susceptible to the plasma environment of space, protection against plasma should be part of the design and it should be tested in a plasma environment. A test for this purpose has been developed (Reference 16). A simulated plasma environment is generated by ionizing the residual gases in the test chamber with an electron beam.

\section{Testing Problems}

The foremost questions confronting the engineer with the responsibility for testing high voltage circuitry are what tests to perform and what instrumentation to use. These questions have only partial answers. Failures that cannot be duplicated in ground testing still occur in space. Some typical tests are outlined in the JPL specification, along with some answers to the instrumentation problems (Reference 17). A wealth of information exists on high potential testing but it is directed to phenomena and operation at atmospheric pressure.

Even the normally simple task of applying a voltage to the test item can become very complicated. The connectors and cabling must be breakdown free. Special connectors and vacuum feed-throughs must be used and these are normally good only at moderate voltages $(150 \mathrm{v})$. The best results are obtained when the high voltage supply is also in the region of reduced pressure; the high voltage leads can then be hard wired to the test item. However, the power supply must first be made discharge free. When testing a circuit with multiple high voltage supplies, it is recommended that separate low voltage supplies be used so that discharge transients or noise are not coupled directly to the other high voltage circuitry.

If exposed high voltage terminals are required, the breakdown strength will depend on the polarity of the high voltage, and this should be taken into consideration. If possible, the cathode of open photomultiplier tubes should be at ground potential. On several occasions false counting or noise has been produced in sensitive experiments by particles from the ion gauge or alphatron gauge on the vacuum system; consideration should be given to this if unexplained data are received during testing.

The normal problems associated with bench testing also occur in vacuum, e.g., ground loops (at the feed-through), and ac power line noise and transients. 


\section{Sample Specification}

This section consists of a reproduction of a design requirement specification for high-voltage protection of the flight equipment for Mariner Mars 1969. This document was prepared by the Jet Propulsion Laboratory and has been made available for this publication through the good offices of $\mathrm{Mr}$. Earle $\mathrm{R}$. Bunker, Jr. It is included here as an illustration of the nature of the specification required to insure the procurement of high voltage equipment that is adequately protected against electrical breakdown failures. Naturally, specifications for different systems will differ in details, but this particular specification is a good example of the kind and amount of detail that needs to be included.

The testing program, which is called out in Section 4 of the JPL document, Quality Assurance Provisions, represents an added cost in the development of flight hardware. The tests should be done on subsystems as indicated. If such tests were done at Goddard Space Flight Center, a reasonable estimate places the cost per test at about $\$ 15,000$. The additional cost for a complete spacecraft development can be arrived at by determining the number of subsystems that will require such tests and the number of models of each subsystem to be tested and applying the above estimate to the total number of tests. 
DESIGN REQUIREMENT

MARINER MARS 1969 FLIGHT EQUIPMENT HIGH VOLTAGE PROTECTION 


\section{SCOPE}

1.1 Scope. This document covers the design requirements for providing High Voltage Protection for Mariner Mars 1969 Flight Equipment.

1.2 Description. The High Voltage Protection requirements delineated herein are to be applied to packaging and testing of electronic equipment operating within a specified voltage range. Voltage breakdown considerations not included in this document are as follows:

a. Voltage breakdown in cavities or wave guides in vacuum due to secondary emission (multipacting) or other effects.

b. Conduction (Townsend) current between two electrodes of the order of $10^{-8}$ ampere or less due to the existence of free electrons in the gap.

High voltage protection requirements a re applied to equipment design to assure that electronic equipment employing high voltages will survive an intentional or inadvertent turn-on and operate without damage while in the critical air pressure region or the vacuum of space.

\section{APPLICABLE DOCUMENTS}

2.1 The following documents, of the exact issue shown, form a part of this design requirement to the extent specified herein.

\section{SPECIFICATIONS}

Jet Propulsion Laboratory

FS500441 B

Soldering Process, Mariner Mars 1969 Spacecraft, General Specification for 
*FS500450

Transformers and Inductors, Electronic Packaging, General Specification for

Military

MIL-T-27B Transformers and Inductors (Audio, Power, and High

Power), General Specifica-

tion for

\section{REQUIR EMENTS}

Jet Propulsion Laboratory

M69-3-220

Functional Requirement,

1 November 1966 Electronic Packaging

$*$ M69-220-1

Design Requirement, Mariner Mars 1969 Flight Equipment, Electronic Packaging

M69-220-5A

3 May 1967

Design Requirement, Planer Packaging, Mariner Mars 1969 Spacecraft

STANDARD

Military

MIL-STD-202C
Military Standard, Test
Methods for Electronic and
Electrical Component Parts

PROJECT DOCUMENT

Jet Propulsion Laboratory

PD 87

Mariner Mars 1969 Parts

13 October 1966

Control Document

*Not released as of the release date of this design requirement. 


\section{REQUIREMENTS}

3. 1 Conflicting requirements. In case of conflict between the requirements of this document and the requirements of any document referenced herein, the conflict shall be referred to the procuring activity or contracting officer for resolution.

3. 2 General. All electronic equipment to be exposed to the critical pressure region as defined in 6.1 .3 and employing voltages in the range specified in 3.2 .1 and frequencies specified in 3.2 .2 shall comply with the requirements of this document and JPL Design Requirement M69-220-1. Electronic equipment shall conform to the applicable packaging requirements of JPL Functional Requirement M69-3-220.

3.2.1 High voltage limits. The requirements of this document are applicable to spacecraft equipment employing instantaneous voltage differences between $250 \mathrm{v}$ and $100 \mathrm{kv}$ for discrete frequencies or waveforms with fundamental frequency components between dc and $100 \mathrm{MHz}$. The lower voltage limit at frequencies higher than $60 \mathrm{~Hz}$ shall be reduced in accordance with Figure 1. Voltages shall be measured between conductors and between each conductor and the subchassis or common metallic ground, using equipment capable of measuring instantaneous values.

3.2.2 Frequency range. The range of frequencies required in equipment design shall be from dc to $100 \mathrm{MHz}$.

3.2.3 Separate chassis grounding leads. Chassis grounding leads shall be separate from signal and power return leads. Provisions shall be made so that corona detection networks may be inserted in series with power grounding leads during subsystem tests, as shown on Figure 2.

3. 3 Classes of electronic equipment. Electronic equipment shall be classified as Class 1 or Class 2 in accordance with 3.3 .1 and 3.3.2. 


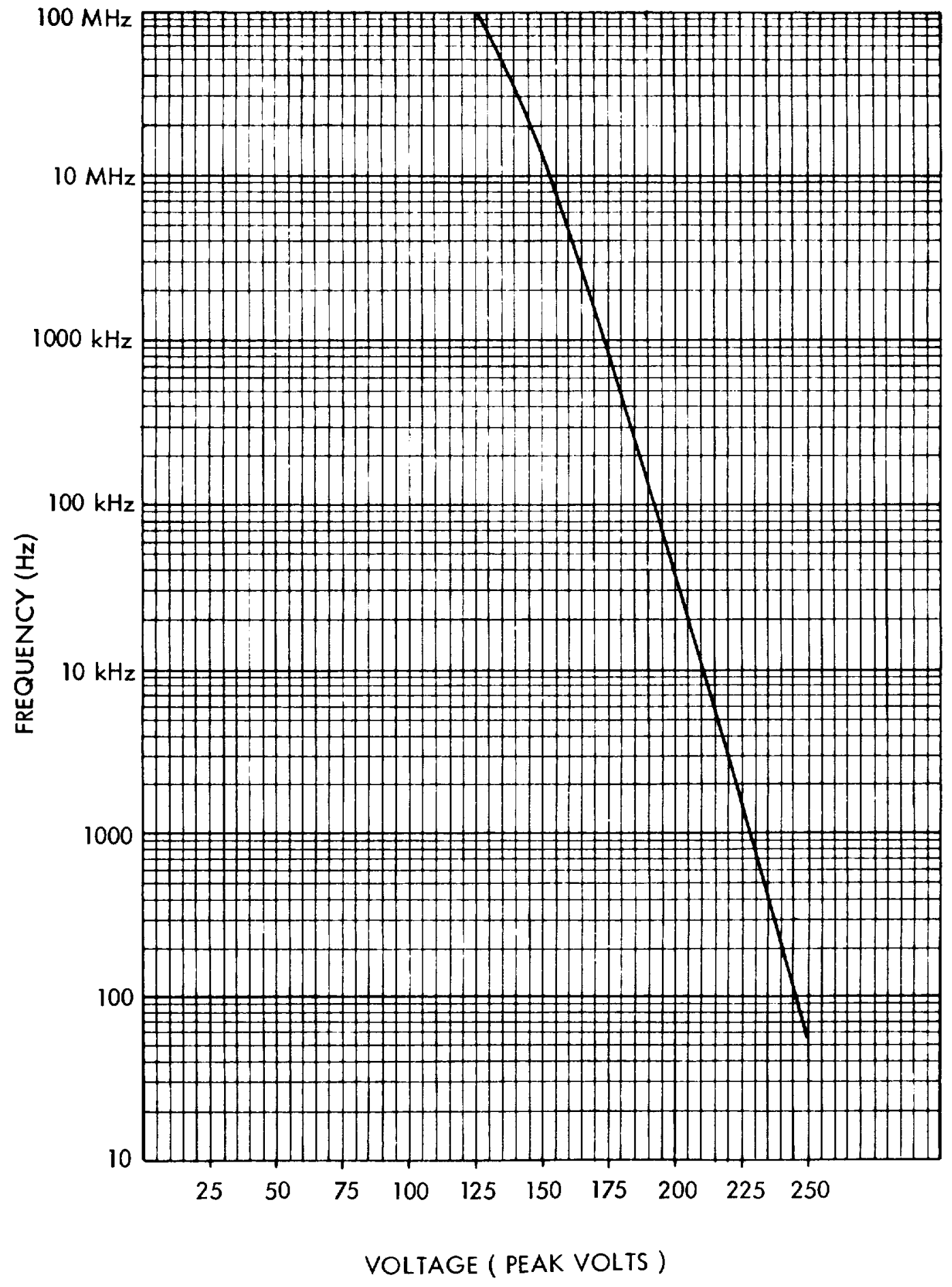

Figure 1. Lower Voltage Breakdown Limit versus Frequency 


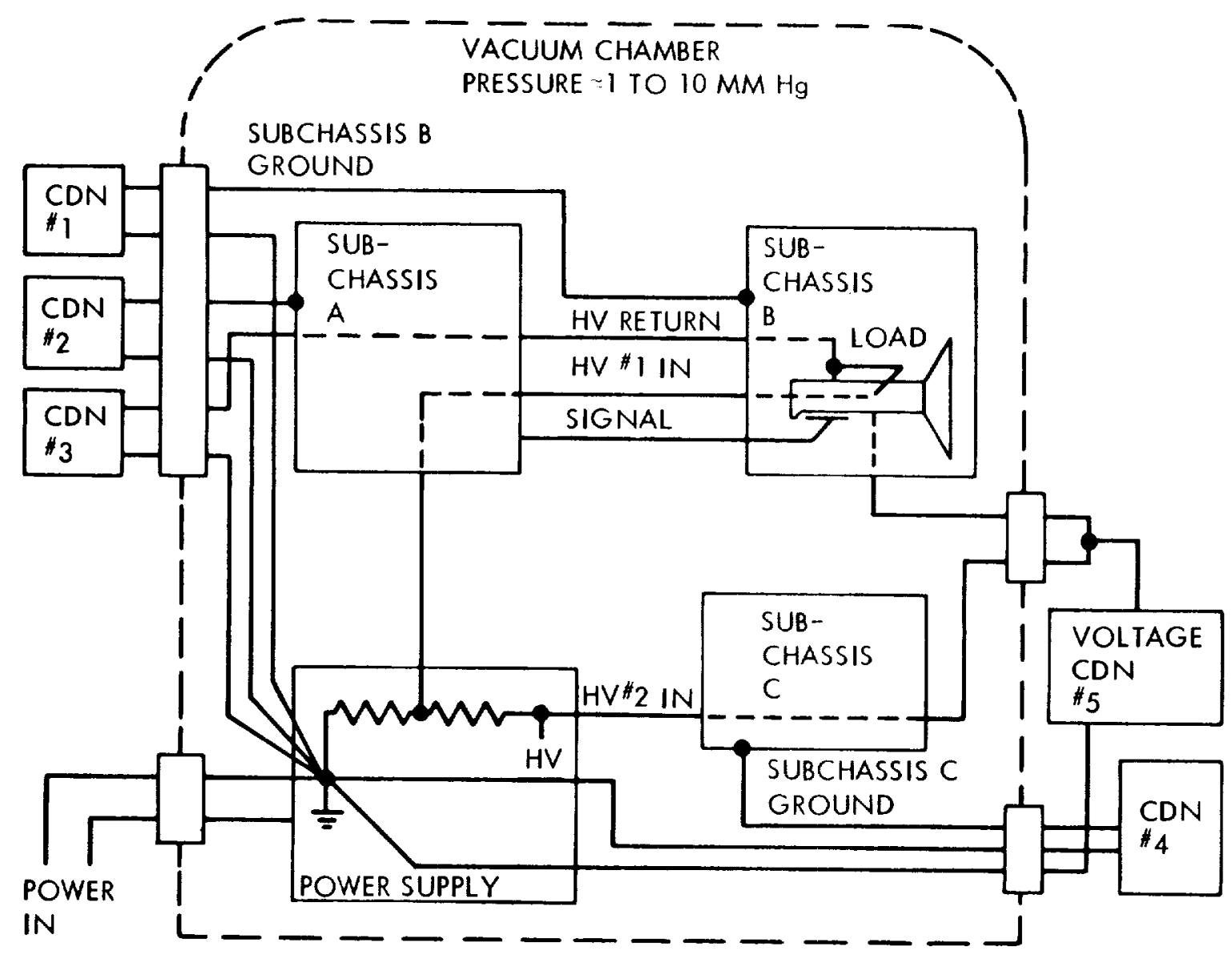

NOTES:

1. CDN = CORONA DETECTION NETWORK. (SEE FIGURE 3).

2. CDN 1, 2, 4 MONITOR POSSIBLE CORONA CURRENTS FROM HIGH VOLTAGE LINES TO CORRESPONDING SUBCHASSIS. CDN 3 MONITORS POSSIBLE CORONA CURRENTS BETWEEN HV \#I CONDUCTOR AND RETURN.

3. IN CASES WHERE IMPEDANCE IN RETURN OR CDN ADVERSELY AFFECTS SUBSYSTEM OPERATION, VOLTAGE TYPE (CON \#5) MAY BE CONNECTED TO HV LEAD ("2) AS SHOWN.

4. ONE CDN WITH GROUNDING WHITCH COULD BE USED IN PLACE OF $\operatorname{CDN} 1,2,3,4$.

Figure 2. Insertion of Corona Detection Networks in Subsystem Ground Returns 
3.3.1 Class 1 equipment. Class 1 equipment shall be designed to operate to specification requirements throughout its operational lifetime without voltage breakdown (arcing or corona) present at any pressure. This prohibition of arcing or corona shall not preclude the use of equipment or components which require arcing or corona as a proper functional requirement (e.g., spark gaps).

3.3.2 Class 2 equipment. Class 2 equipment shall be designed so that any voltage breakdown which may appear during operation at any pressure shall not cause damage to its internal components or to other external equipment.

3.3.2.1. Protective devices. In Class 2 equipment applications where insulation to prevent corona or arcing cannot be used because of interference with the proper functioning of the unit (e.g., plasma detector screens), protective devices such as horn gaps shall be used to reduce the possibility of arcing or corona occurring in the unit. Power supply output shall be self limiting to prevent damage due to arcing or corona.

3. 4 High voltage circuitry requirements.

3.4.1 Printed wiring and terminal boards. Printed wiring and terminal boards used in high voltage circuits shall meet the requirements of 3.4 .1 .1 through 3.4 .1 .6 .

3.4.1.1 Separation of high voltage circuits. Circuits employing high voltage shall be physically separated from low voltage circuits when located on a printed wiring board. The minimum distance between high and low voltage conductors shall be as given in 3.4.1.2 and 3.4.1.3.

3.4.1.2 Spacing of conductors. The distance between conductors, terminals, or other metallic surfaces shall be measured from the points of closest approach, including worst tolerance buildup, and disregarding any intervening insulation materials.

3.4.1.3 Conductor separation. The minimum separation of printed conductors carrying voltages in accordance with 3.2 .1 on the same side of the printed wiring board 
shall be as given by the empirical equation $d=0.250 \sqrt{\mathrm{V}}$ where $d$ is in inches and $V$ is the maximum peak voltage difference between the conductors in kilovolts. Distances shall be measured as in 3.4.1.2.

3.4.1.4 Spacing from edge. The minimum distance of the conductors from the edge of the board shall be 1.5 times the value obtained from the equation in 3.4 .1 .3 .

3.4.1.5 Insulation thickness. High voltage conductors on a printed wiring board bonded to a metallic subchassis shall have a minimum total insulation thickness equal to that specified in 3.5.3. Selection of insulating material and bonding procedures shall be in accordance with JPL Design Requirement M69-220-5 A. If swaged terminals are used in the high voltage circuit, the insulation thickness shall be measured between the swaged end and the metallic subchassis in accordance with 3.4.1.2.

3.4.1.6 High voltage pulse circuits. Minimum separation as specified in 3.4.1.3 of conductors carrying pulses may be reduced by the multiplying factor $\frac{t}{t+0.8}$ where $t$ is the pulse width in microseconds. The pulse duty cycle shall be less than five percent for this reduction to apply.

3.4.2 Sharp points. Conductor configurations, electronic parts, and mechanical parts either in the high voltage circuit or insulated electrically but at a distance that is less than twice the distance specified in 3.4.1.3 from the high voltage conductors, shall be designed or laid out in a manner that shall avoid sharp points, corners, and abrupt changes in dimensions. Solder fillets shall be smooth.

3.4.3 Solder terminals. Solder terminals shall be kept to a minimum in high voltage circuits. Terminals with sharp points, edges, or abrupt changes of dimensions shall be avoided unless such points, edges, etc, are radiused and filleted with solder to obtain smooth surface transitions. Installation of electronic parts shall conform to Design Requirement M69-220-5 A except that part leads shall not protrude beyond the solder terminals. 
3.5 High voltage insulation material requirements. High voltage insulation requirements for materials shall be as specified in 3.5.1 through 3.5.11.

3.5.1 Dielectric strength. Insulating materials having the higher dielectric strengths shall be used in high voltage applications when other properties or characteristics pertinent to the application are similar. Materials with normal dielectric strengths of less than 400 volts per mil at the thickness required should be avoided.

3.5.2 Dielectric constant. Insulating materials with low dielectric constants shall be selected for insulation of ac voltages. Where two different insulating materials are in contact, they should be selected so that the difference in their dielectric constants is minimum. Materials with dielectric constants greater than 5 should be avoided.

3.5.3 Insulation thickness. The thickness of insulation shall be such that the maximum voltage stress will be 40 volts per mil thickness, or 10 percent of the actual breakdown voltage for the thickness of insulation used in the design, whichever voltage stress is smaller.

3.5.4 Air dielectric strength. For purposes of equipment design in accordance with this specification, air shall be assumed to have a zero dielectric strength in the critical pressure region.

3.5.5 High frequency applications. Insulation materials selected for use in high frequency (nominally above 1. $0 \mathrm{MHz}$ ) applications shall have dielectric constants and dielectric losses small enough so that blistering, delamination, or other internal damage caused by internal heating will not occur during normal operation.

3.5.6 Foams. Expanding or syntactic foam materials utilizing entrapped air or gas for fillers shall not be used for high voltage insulation applications.

3.5.7 Low arc resistant materials. Organic insulating materials which have a tendency to sustain arcing under any pressure condition or which deteriorate or outgas 
under arcing conditions shall not be used in contact with bare conductors emerging from the insulating material and exposed to the ambient air pressure. Inorganic insulating materials which do not sustain arcing shall be used to provide the interface of an emerging bare conductor from the embedment or conformal coating.

3.5.8 Filled materials. Insulation which employs fillers or discrete materials mixed throughout its volume shall have a dielectric strength design limit within the requirements of 3.5 .3 computed for that material of the mixture with the lowest dielectric strength.

3.5.9 Insulation coating. All exposed conductors carrying high voltages shall be conformally coated or embedded in a plastic material in conformance with this specification. Conductors which must be exposed to the ambient air pressure for proper functioning (e.g., spark gaps) shall be exempt from this requirement.

3.5.10 Adhesion of polymeric materials. Selection of polymeric insulation materials and preparation of solid surfaces in contact with such materials shall be such as to ensure proper adhesion of the polymeric materials to eliminate creepage paths between conductors.

3.5.11 Removal of absorbed air. Insulation materials in liquid form prior to polymerization shall be exposed to a vacuum sufficient to remove entrapped air. Suitable precautions shall be taken to prevent re-entry and trapping of air into the insulation material prior to use and during application.

3. 6 Enclosures. Enclosures shall be vented directly to the ambient vacuum of space. The total area of vent openings shall be such as to allow the pressure in the enclosed volume to bleed down to $3 \times 10^{-3}$ millimeter of mercury in 60 seconds or less when the pressure is reduced from ambient sea level to $10^{-5}$ nillimeter of mercury in 6 seconds or less. The pressure referred to includes both residual air and outgassing in the enclosure. 
3. 7 Electronic parts for high voltage applications. addition to the requirements specified herein and in JPL Project Document 87, electronic parts used in high voltage circuits shall be selected in accordance with component and subsystem requirements and accepted design practices.

\subsubsection{Transformers and inductors. Transformers} and inductors shall meet the requirements of JPL Specification FS500450 and 3.7.1.1 through 3.7.1.5.

3.7.1.1 Maximum voltage between turns. The thickness of the wire insulation coat and winding technique shall be such that the maximum possible voltage between any two adjacent wires in a winding shall be in accordance with 3. 5.3 and in no case larger than 40 volts peak. Voltages at terminations of windings and between wires in excess of this value shall employ additional insulation in accordance with 3.5.3. In high voltage pulse transformers with pulse widths of $10 \mu \mathrm{sec}$ or less, the allowable voltage limit between wires may be 200 volts peak.

\subsubsection{Core connection. Electrically conductive} cores electrically insulated from the mounting base of the transformer or inductor shall have an auxiliary lead brought out to facilitate hypot tests between the core and the windings to test core insulation integrity per 4.4.3.2, if there is no internal connection between a winding and the core. Cores fabricated from high permeability magnetic materials and encased in plastic material by the manufacturer are exempt from this requirement if the plastic material is in accordance with 3.5 and is sufficiently transparent to allow measurement of the minimum thickness of insulation separating the core from the windings. Cores encased with metallic covers and covered with insulation material are also exempt if the insulating material is in accordance with 3.5 and the low voltage winding (primary) is between the high voltage windings and the core and covers the core completely.

3.7.1.3 Interwinding insulation. Insulation between windings shall be in accordance with 3.5 and capable of withstanding, without damage, the tests described in 4.4.3.2. 
3.7.1.4 Winding embedment. Windings shall be impregnated and then encapsulated or errbedded with suitable materials and techniques in accordance with 3.5 so that all wires are securely anchored and no pockets or voids occur.

3.7.1.5 Winding terminations. Windings terminated into insulated lead wires shall be embedded in accordance with 3.7.1.4 and the requirements of this paragraph. Terminals employed for termination of transformer or inductor windings shall meet the requirements of 3.4 .3 prior to conformal coating or encapsulating in accordance with 3.5. The conformal or encapsulant material shall be compatible with the lead wire insulation and achieve a thorough bond so that creepage paths will not occur. The length of bond shall be 0.25 inch or in accordance with 3.4.1.3, whichever is greater.

3.7.2 Connectors. Connectors shall meet the requirements of 3.7 .2 .1 through 3.7 .2 .3 .

3.7.2.1 Venting. The void enclosed between the interface of the mated connector, and other volumes sealed off by cable clamps, etc., shall be vented in accordance with 3.6 .

3.7.2.2 Insulation. The insulation material shall be selected in accordance with 3.5 .

3.7.2.3 Class 1 application. Connectors shall not be used as high voltage interfaces in Class 1 equipment unless compliance of such connectors with the requirements of this specification is demonstrated by suitable tests.

3.8 Performance. The performance of high voltage parts and components shall be substantiated by testing as specified in Section 4 . Test objectives shall be in. accordance with 3.8 .1 and 3.8 .2 .

3.8.1 Subsystems. The principal objective of operating a subsystem employing high voltage in the critical region, as a part of Type Approval testing, shall be to demonstrate the capability of the subsystem to survive operation in this environment. An additional objective shall 
be to qualify details of design such as (1) design concept, (2) adequacy of interconnections, (3) effectiveness of protective devices, (4) effect on other subsystems, and

(5) quality of workmanship.

3.8.2 Electronic parts. The objectives of high voltage tests of components shall be to prove the thoroughness of the internal design, adequacy of insulation, suitability of materials used, and quality of workmanship.

3.9 Workmanship. All parts and components intended for high voltage usage shall be manufactured to a high standard of workmanship. Uniformity of shape, dimension, and construction shall permit interchangeability of replaceable parts and components. There shall be no cracks, breaks, chips, bends, burrs, loose attachments, illegible markings, or other evidence of workmanship defects which could adversely affect the performance or life of parts and components.

\section{QUALITY ASSURANCE PROVISIONS}

4.1 General. Inspections and tests as specified herein shall be performed on all parts and components used in high voltage applications to substantiate the requirements of Section 3.

4. 2 Electronic part type qualification. Electronic parts for high voltage applications shall be exposed to test conditions sufficiently severe in the critical pressure region to establish margins and test the design construction and types of insulation materials used. The test conditions shall be severe enough to cause some degradation or failure so that the margin between normal operation and the test conditions can be determined. Specific test levels shall be determined from appropriate part specification documents.

4.3 Electronic part acceptance. One hundred percent of all electronic parts that have high voltage applications in flight equipment shall be operated at increased voltages with suitable corona detection networks to prove the adequacy of each electronic part for operation in the critical region. Excess voltages applied to flight electronic parts shall not 
be large enough to cause deterioration or damage. Acceptance tests of electronic parts used in a subsystem shall not eliminate the requirement for operational tests at critical subsystem pressure. Specific test levels shall be determined from appropriate part specification documents.

4. 4 Electronic part testing. All electronic parts used in high voltage circuits shall be tested for the designated period of time in accordance with the type of test and test setup specified. These test conditions shall be applicable to all electronic parts except those specially named under appropriate paragraphs of this specification. Applicable paragraphs of MIL-STD-202 C, Method 301, which are not in conflict with this specification, may be used as a guide for electronic part tests.

4.4.1 General electronic part design qualification. Electronic parts used at high voltages shall be tested for corona or arcing in the critical pressure region to determine the adequacy of construction of the electronic part from high voltage considerations. Following suitable precautions to minimize external corona and arcing, the voltages applied shall be while the electronic part is in the critical pressure region. It is expected that applied voltages in design qualification tests will be high enough to cause some failures, in order to establish a margin of confidence. Voltages applied to electronic parts shall be greater than normal operating voltages in order to accelerate failure of marginal or defective components. For convenience in testing, a functional group of electronic parts which form a component of the subsystem may be tested in accordance with this specification (rather than each electronic part separately).

4.4.1.1 Test procedure. Test voltáges as given in the respective paragraphs shall be applied to the electronic parts or components undergoing test in a vacuum chamber at room pressure. Corona detection networks as shown on Figure 3 shall be used in appropriate leads to monitor for corona or arcing. With voltage continuously applied, the air pressure shall be reduced to the lower limit defined in 6.1.3 and this pressure varied between the upper and lower limits several times for the time interval specified in 


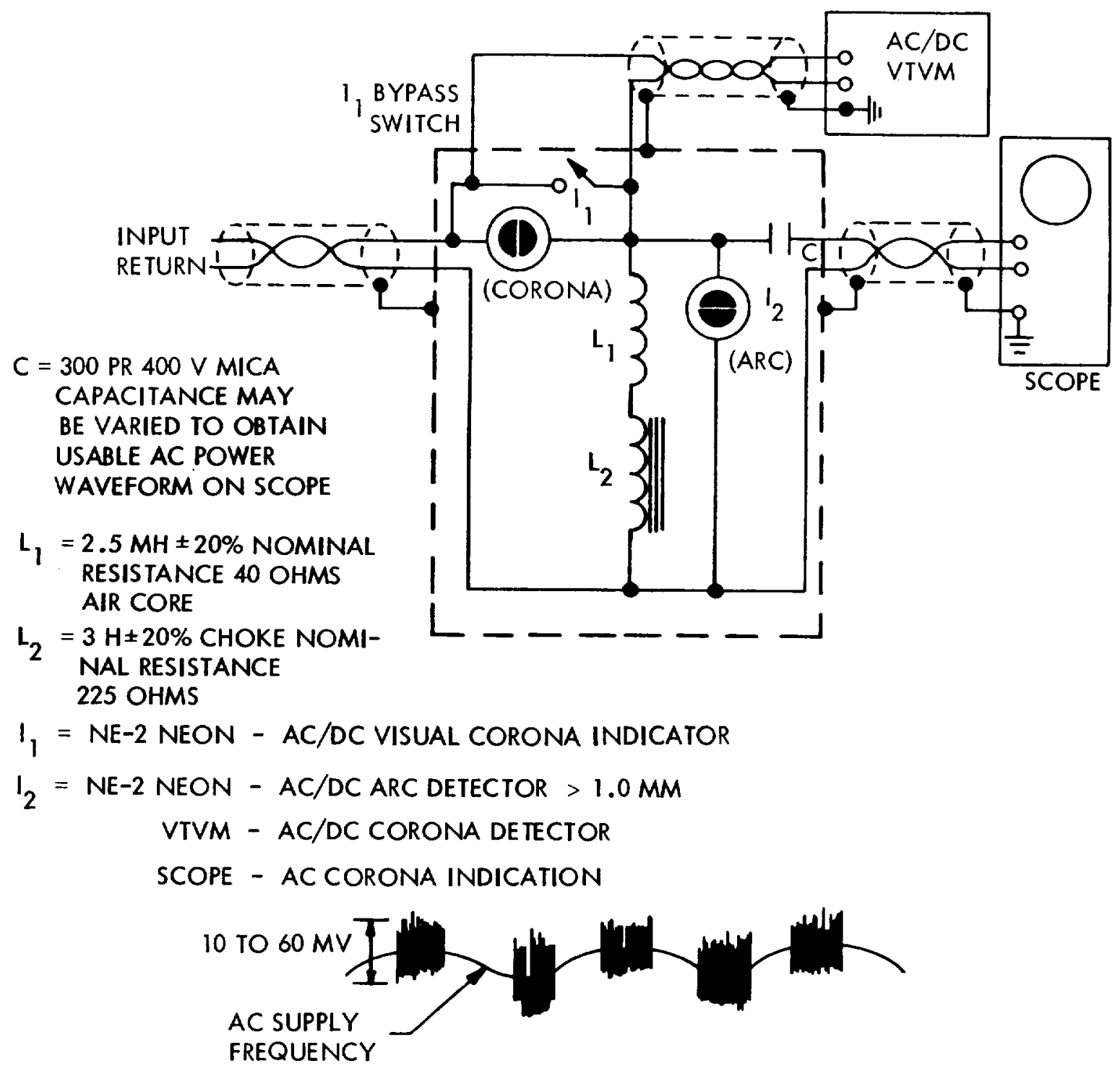

NOTES: 1. IF ELECTRODE CONFIGURATION IS UNSYMMETRICAL, CORONA BURSTS ON ONE POLARITY OF SUPPLY FREQUENCY WAVEFORM MAY BE ABSENT.

2. ABRUPT BREAKS IN SCOPE TRACE OR BURST AMPLITUDES $>0.5$ VOLT PEAK-TO-PEAK INDICATE ARCING, RATHER THAN CORONA.

3. MINIMUM SENSITIVITY $10 \mathrm{MV} / \mathrm{CM}$.

Figure 3. Corona Detection Network Schematic 
4.4.1.7. If several voltage tests are to be made on the same electronic part or component or on several in the same chamber, the test voltages may be applied in sequence by switching, if the chamber pressure is varied between the limits of 6.1.3 for each test. At the conclusion of the test, the voltage shall be removed and the electronic part or component brought back to ambient room pressure. During the test, evidence of corona or arcing shall be cause for rejection. Voltage lines and feed throughs to the component in the test chamber shall be of such construction as to preclude the formation of corona.

4.4.1.2 Test mounting. Electronic parts or components undergoing test shall be mounted in a similar manner to that in the subsystem, especially with regard to adjacent metallic surfaces, terminals, etc. Potting, coating, or encapsulation shall be similar to that applied to the electronic part or component in the complete subsystem.

4.4.1.3 Test voltage amplitude. The test voltage shall be twice the maximum operating voltage. If both ac and dc voltages are applied simultancously to an electronic part or component during normal operation, then the test voltage shall be an ac sine wave with a peak amplitude equal to twice the sum of the $\mathrm{dc}$ and peak ac operating voltages. If it can be shown that application of the lest voltage specified in this paragraph will exceed the manufacturer's ratings, then the test voltage may be reduced to 130 percent of maximum operating voltage upon written approval of JPL.

4.4.1.4 Test voltage frequency. The frequency of the test voltage shall be within plus or minus 10 percent of that experienced by the electronic part of component during normal operation. DC voltages shall not be applied to electronic parts or components normally operating at ac potentials. Electronic parts or components which normally operate on dc voltages shall be tested by the application of a dc voltage in accordance with 4.4.1.3 Electronic parts or components which normally operate on ac shall be tested by sine wave voltages of a peak amplitude as specified in 4.4.1.3. If more convenient, a $60 \mathrm{~Hz}$ ac sine wave instead of the normal operating frequency may be used, subject to the restriction that the $60 \mathrm{~Hz}$ test frequency shall not be 
nearer than plus or minus 20 percent of the resonant frequency of the component. Such a test frequency shall not cause damage to the component, and that the normal operating frequency of the component is under $6.0 \mathrm{kHz}$.

4.4.1.5 Test voltage application. Voltages shall be applied between terminals of the electronic part of component. If the terminals are insulated from the metallic case or mounting hardware, then the test voltage shall also be applied between the terminal and the case or the mounting hardware.

4.4.1.6 Rate of voltage application. The test voltage shall be raised uniformly from nominally zero to the final value at a maximum rate of 500 volts per second, dc or rms, unless otherwise specified.

4.4.1.7 Test duration. The test voltages shall be applied in accordance with applicable paragraphs of this specification for the minimum length of time of 1.0 hour in the critical pressure region.

4.4.2 General electronic part acceptance tests. Acceptance tests at sea level conditions of electronic parts for flight subsystems shall be performed on a 100 percent rather than sampling basis to screen out electronic parts with defective workmanship or concealed damage, in accordance with 4.3 .

4.4.2.1 Operating test voltage. The voltage applied between the terminals of an electronic part shall be 130 percent of the manufacturer's rating for the test time specified in 4.4 .2 .5 .

4.4.2.2 Insulation test voltage. Transformers with graded insulation shall be exempt from the requirements of this paragraph. In transformers without graded insulation, voltages applied between all the terminals tied together and mounting hardware shall be of sufficient magnitude to stress the dielectric at the narrowest section to 80 percent of the rated dielectric strength of the insulation material for the period of test time specified in 4.4.2.5. Components with no conducting mounts or enclosure shall be buried in 
metallic shot or have a conducting foil wrapped on the surface of the insulation to serve as the voltage return. Corona detection networks shall be used to monitor possible corona or arcing.

4.4.2.3 Frequency. The frequency of the applied voltage shall be the same as that under normal operating conditions. If this is not practical then 4.4.1.4 shall apply.

4.4.2.4 Rate of application. The rate of application of test voltages may be instantaneous. The minimum rate shall be as required by 4.4 .1 .6 .

4.4.2.5 Test duration. The minimum time for the full voltage to be applied to the electronic part shall be $5.0 \pm$ 0.5 seconds.

4.4.3 Transformer/inductor tests. All transformers and inductors shall be tested in accordance with 4.4 .1 , where applicable. The tests specified in 4.4.3.1 through 4. 4.3.5 shall be a requirement for acceptance prior to installation in a subsystem. Electronic part acceptance tests as well as design qualification tests on transformers and inductors shall be performed at critical pressures.

4.4.3.1 Test configuration. The configuration for testing transformers and inductors shall be as shown on Figures 4 and 5. Electrical connections shall be corona proof when the pressure is in the critical region.

4.4.3.2 Interwinding insulation. The insulation integrity between windings, between a winding and the core, and between a winding and the case if one is used, or between windings and mounting inserts if used, shall be tested by applying a voltage between the various windings, cores, etc., in accordance with Figure 4 and the following table for the length of time specified in 4.4.2.5.

Table I. Interwinding Insulation Test Voltages

\begin{tabular}{|c|c|}
\hline $\begin{array}{c}\text { Working Voltage } \\
(\mathrm{dc} \text { plus peak ac) }\end{array}$ & $\begin{array}{c}\text { Test Voltage } \\
(\mathrm{rms})\end{array}$ \\
\hline $250 \mathrm{v}$ to $700 \mathrm{v}$ & $2.8 \times$ \\
\hline Above $700 \mathrm{v}$ & $1.4 \times$ plus 1000 \\
\hline
\end{tabular}




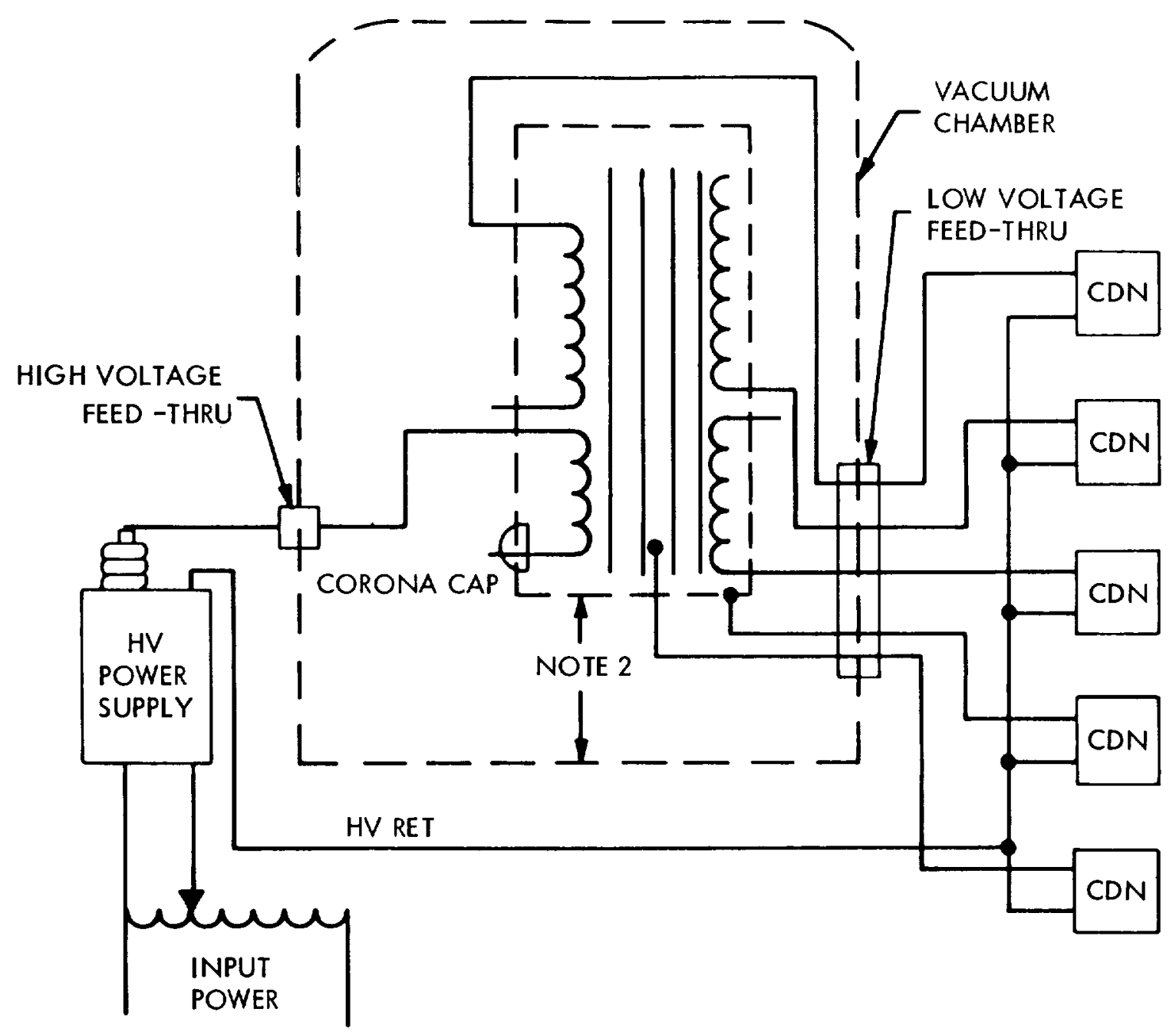

NOTES: 1. GROUNDING TYPE SELECTOR SWITCH MAY BE USED WITH ONE CORONA DETECTION NETWORK.

2. TRANSFORMER MUST BE ELECTRICALLY INSULATED (LOW VOLTAGE) FROM VACUUM CHAMBER GROUND.

3. $C D N=$ CORONA DETECTION NETWORK (SEE FIGURE 2).

Figure 4. Transformer Interwinding Breakdown Test 


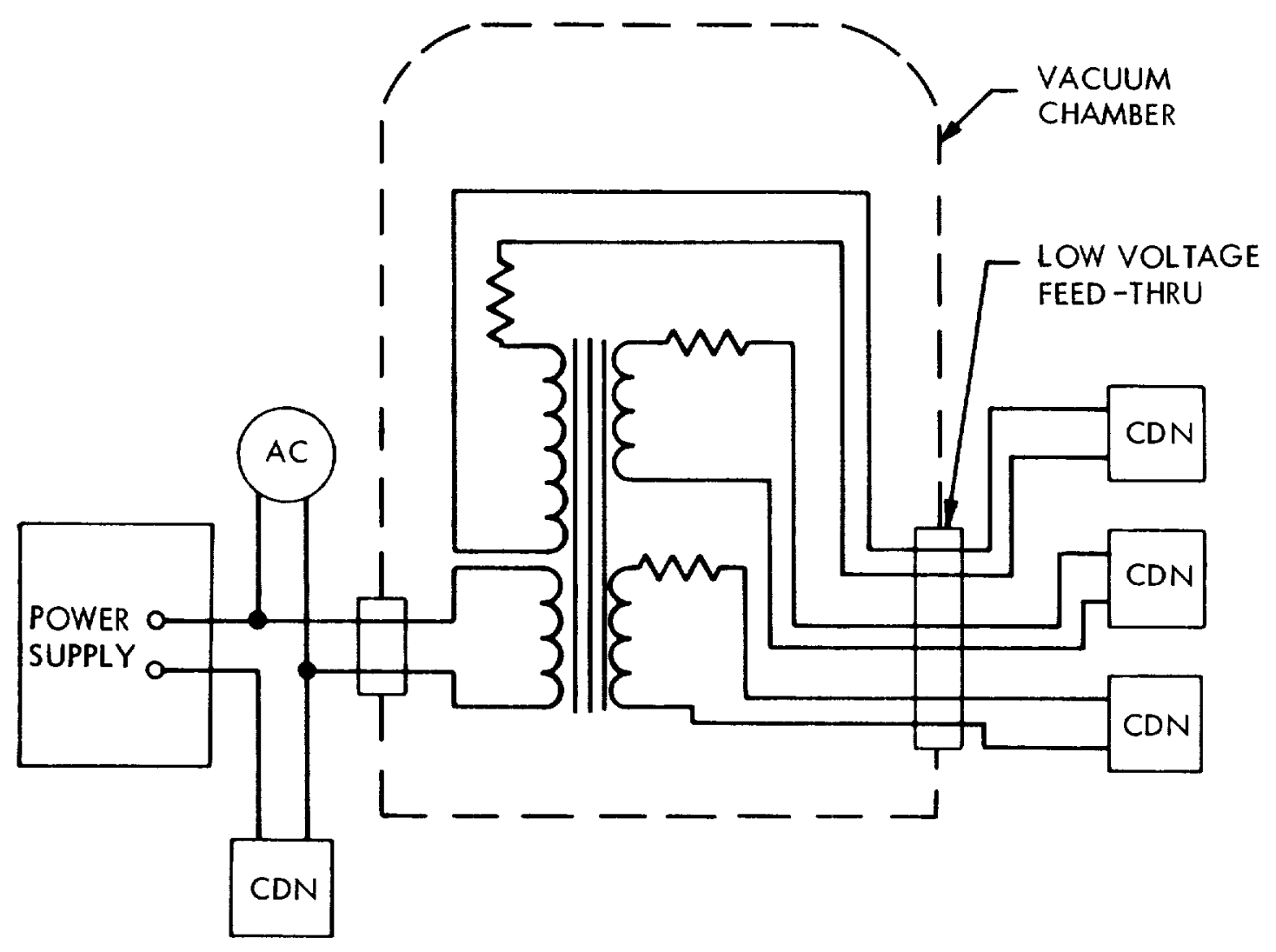

NOTES: 1. RESISTORS ARE LOADING R'S FOR SECONDARY WINDINGS.

2. NEON INDICATOR I , SHALL BE BYPASSED FOR THIS TEST.

3. POWER SUPPLY VOLTAGE SHALL BE TWICE RATED VOLTAGE FOR THE WINDING ENERGIZED WITH THE FREQUENCY RAISED SO THAT AC CURRENT FLOW IS EQUAL TO OR LESS THAN RATED CURRENT.

4. GROUNDING TYPE SELECTOR SWITCH MAY BE USED WITH ONE CORONA DETECTION NETWORK.

5. CDN = CORONA DETECTION NETWORK (SEE FIGURE 2).

Figure 5. Transformer Intrawinding Breakdown Test 
4.4.3.3 Intrawinding insulation. Transformers shall be subjected to a voltage sufficient to cause twice the rated voltage to appear across all windings at the critical pressure. The test voltage may be applied to any winding as shown on Figure 5. Care shall be taken to terminate all transformer terminals so that external corona or arcing is prevented. Mountings and windings shall be grounded as they would be in service. The applied frequency shall be at least twice the normal operating frequency so that rated current is not exceeded. The test frequency shall be remote from any resonant frequency. Twice the rated voltage shall be applied across an inductor winding at approximately twice the normal frequency, or in a manner that will not exceed rated current.

4.4.3.4 Test duration. Test duration requirements shall be as follows:

a. Design qualification: Test duration for component design qualification tests - shall be as specified in 4.4.1.7.

b. Component acceptance: Test duration for component acceptance shall be as specified in 4.4 .2 .5 .

4.4.3.5 Examination during and after test. Components undergoing tests as described in the previous section shall show no internal corona or arcing during the test. After the tests, components shall be examined for evidence of arcing, flashover, breakdown of insulation, and damage. Visible damage or detection of voltage breakdown or corona by instrumentation shall be cause for rejection.

4. 5 Test equipment. Test equipment requirements shall be as follows.

4.5.1 Corona detection network. Detection of corona or arcing shall be by a current or series type of network, as shown on Figure 3, inserted in series with the ground or return of the high voltage circuit being tested. In cases where the added series impedance in the return is detrimental to the operation of the component or system, the 
parallel or voltage type described in MIL-T-27B shall be used. Selection of the type of detection network used shall be based on the following criteria.

a. The current or series type shown on Figure 3 , composed of low voltage rating elements in the ground or low voltage return side of the circuit, enables isolation of the returns so that the location of the corona may be determined.

b. The high voltage or shunt type is connected directly to the high voltage terminal through a high voltage corona-free blocking capacitor. Corona occurring in this series network will be indistinguishable from that occurring in the equipment undergoing tests.

In places where connections are made inside the vacuum chamber as described in 4.5.3, the current type of corona detection network makes it possible to distinguish between corona externally at a component terminal, and internally through the case insulation. Corona occurring at a terminal in a vacuum chamber, which is not a defect of the component, shall not abort the test by preventing the detection of corona occurring in the component. The parallel type described in MIL-T-27 B does not have this capability.

4.5.1.1 Network ac operation. The current type corona detection network shall be inserted in series with return or ground lines to monitor the current flowing in the circuit. Indicators I- 1 and I- 2 shown on the Figure 3 schematic, serve the dual purpose of corona and arc indication, and overvoltage protection. Inductance L- 1 and L-2 in series provide a significant ac impedance from audio frequencies to nearly $0.5 \mathrm{Mc}$ respectively, which is the significant frequency range of corona voltage. The function of the capacitor $\mathrm{C}$ is to attenuate the ac supply frequency to a sufficient degree, but pass the corona burst pulses so that the maximum sensitivity of the oscilloscope may be utilized. The power supply waveform appearing on the oscilloscope shall serve as a reference for corona bursts, as shown by the waveform of Figure 3. Corona 
bursts can thus be distinguished from extraneous noise in the circuit.

4.5.1.2 Network dc operation. In dc operation, capacitor $\mathrm{C}$ will attenuate power supply ripple or low frequency noise. The high frequency corona burst may or may not appear on the oscilloscope depending on the geometry, voltage, polarity, and other factors. DC corona indication may also be a continuous smear rather than a burst. If no indication is apparent on the oscilloscope trace, then the neon indicator I- 1 shall be used to monitor for dc corona. If the voltage drop due to indicator I- l cannot be tolerated, then it shall be bypassed and a high gain VTVM connected across L- 1 and L-2 to monitor for dc corona. If appreciable dc is passed through L-1 and L-2 due to the power supply, then corona current fluctuations may be picked up by the oscilloscope.

4.5.2 Vacuum chamber. The vacuum equipment shall have sufficient capacity to pump down to the critical region in 20 minutes with the chamber air and outgassing loads present.

4. 5. 3 Electrical connections. High voltage connections to terminals of equipment inside the vacuum chamber shall be kept to a minimum. Where such connections are unavoidable, they shall be encapsulated in a resilient, easily removable resin using applicable paragraphs of 3.5 as a guide only because of the temporary nature of the connections. To minimize air bubble formation, the requirements of 3.5 .11 shall be followed. After polymerization, a conducting shell of foil shall be wound on the resin and connected to a corona detection network to monitor the connection for corona or arcing.

4.5.3.1 Vacuum chamber penetration. Where possible, to eliminate any terminals in a vacuum, all high voltage connections required through the interface shall consist of insulated wires embedded in the component and long enough to pass through the vacuum port without splicing. Vacuum sealing can be achieved by "O" ring seals under compression around the wire insulation. A continuous pumping system can tolerate the leakage of such seals, 
with the moderate vacuum requirements of the critical pressure region. After acceptance, the component leads can be cut to installation length.

4.5.3.2 Switching. Switching of component or subsystem high voltage leads shall be accomplished external to the vacuun system.

4.5.4 Oscilloscope. The frequency response of the vertical amplifiers of the oscilloscope shall be flat to 1. $0 \mathrm{MHz}$. Deflection sensitivity of the trace shall be 10 millivolts/cm or less. The zerotrace of the oscilloscope shall be blanked out visually by opaque tape so that the intensity can be turned up sufficiently to see the corona bursts.

4.6 Rejection and resubmittal. High voltage parts and components that fail to meet all the requirements of this specification shall be rejected and returned to the contractor. Prior to resubmittal, if applicable, the contractor shall furnish the JPL procurement division representative and the JPL cognizant engineer full particulars in writing regarding the cause of failure, and the action taken to correct the deficiencies.

5. PREPARATION FOR DELIVERY

Not applicable.

6. NOTES

6.1 Definitions. Definitions applicable to this specification are as follows.

6.1.1 Voltage breakdown. Voltage breakdown as used in this specification refers to either arcing or corona.

6.1.1.1 Corona. An incomplete or partial voltage breakdown of the air adjacent to one or both electrodes or conductors, resulting in a current flow of the order of $10^{-7}$ to $10^{-6}$ ampere rms. 
6.1.1.2 Arcing. A complete voltage breakdown of dielectric between two conductors, with currents of the order of milliamperes or higher, limited only by power supply impedance.

6.1.2 Damage. Damage within the requirements of this specification is hereby defined as any degradation, deterioration, or gross change in a circuit component or subsystem that would significantly shorten its operating life or cause an out-of-tolerance change in performance.

6.1.3 Critical pressure region. The range of pressure through which the dielectric strength of the air reduces to 20 percent or less of the dielectric strength at $20^{\circ} \mathrm{C}$ and sea level pressure, shall be the critical pressure region for the purpose of this specification. Nominal limits of the critical pressure region in air are 50 millimeters of mercury $(60,000$ feet altitude $)$ to $5 \times 10^{-4}$ millimeter of mercury (310,000 feet altitude).

(End of JPL Specification) 


\section{DESIGN REVIEW CHECKLIST}

The technique of conducting a design review has been discussed at length in published literature (Reference 18). One of the techniques is the use of a checklist. Though a checklist is not a mandatory approach, appropriate questions are included here for the benefit of those who find such a list helpful. A very extensive list of questions relating to aerospace systems has been published by B. D. Smith and I. M. Aptaker (Reference 19). The list below includes only those questions relating to electrical breakdown which are omitted from the literature or which require special emphasis.

\section{Checklist Questions}

1. Are any hollow core resistors used?

2. If hollow core resistors are used, are they adequately sealed or vented to avoid internal glow discharge?

3. Are any solid state circuit elements so located that voltage gradients (electric fields) of sufficient magnitude to damage them can be imposed in either normal or faulty operation?

4. Are solid state circuit elements protected against damage from excess reverse current in the case of an electrical breakdown?

5. In transformers, chokes, and tuning coils, is the voltage between turns safely below the breakdown threshold?

6. In transformers, chokes, and coils, is insulation adequate to prevent electrical breakdown in the critical pressure region?

7. In transformers, are current limiting design features adequate to prevent loss of function resulting from an electrical breakdown?

8. In connectors and plugs, if used, is adequate venting provided?

9. In connectors and plugs, if used, are the insulating materials chosen for low susceptibility to tracking and treeing?

10. Is the use of connectors and plugs in the high voltage side confined to the minimum number compatible with the intended application?

11. Are large potential gradients possible between exposed adjacent connections on printed circuit boards? 
12. Does the design guard against transients large enough to initiate breakdown in both "make" and "break" operations?

13. Are bleeder resistances incorporated with all capacitors that could store enough energy to cause breakdown?

14. Are current limiting features of all circuits adequate to prevent both self-damage and neighbor-damage in the event of electrical breakdown?

15. Are test points readily accessible for corona detection tests?

16. Can every conceivable electrical breakdown fault be detected by tests?

17. Will catastrophic failure result if test points are grounded?

18. Is cabling insulation adequate for planned operations and for operation through the critical pressure region?

19. Is cabling properly arranged to avoid trapping of gas?

20. Are sharp points and sharp edges completely avoided?

21. Are arc suppression features for both make and break adequate?

22. Are appropriate steps taken to avoid the existence of ions in regions of large potential gradients?

23. Are potting and coating materials chosen so that mechanical stress on parts is kept small?

24. Are the possible effects of aging of potting and coating materials provided for?

25. Are both individual and combined effects of environmental factors such as temperature, vacuum, penetrating radiation, ions, and outgassing taken into account in the design?

26. Are provisions made to prevent inadvertent turnon of high voltage?

27. Has electrical stress analysis been done on all circuits for both normal and breakdown operation? 


\section{ACKNOWLEDGMENTS}

We are indebted to Mr. A. Flanick, of the Goddard Space Flight Center, for the description of the Cockroft-Walton power supply, and to Dr. K. Ogilvie and Mr. J. L. Westrom, both of the Goddard Space Flight Center, for the descriptions of voltage handling to 15 kilovolts. We also thank Dr. J. A. Simpson, Director, and Mr. W. E. Six, Quality Assurance Manager, of the University of Chicago Laboratory for Astrophysics and Space Research, for the specification relating to conformal coating procedure. 


\section{REFERENCES}

1. Llewellyn-Jones, F., "Ionization and Breakdown in Gases," London: Methuen \& Co., Ltd., 1957.

2. Loeb, L. B., "Basic Processes of Gaseous Electronics," 2nd edition, Berkeley and Los Angeles: Univ. of Calif. Press, 1955.

3. Cobine, J. D., "Gaseous Conductors," New York, Dover: 1958.

4. Llewellyn-Jones, F., "The Glow Discharge," London: Methuen and Co., Ltd., 1966.

5. Francis, G., "The Glow Discharge at Low Pressure," Handbook der Physik, Vol. XXII, Berlin: Springer-Verlag, 1956.

6. Brown, S. C., "Breakdown in Gases," Handbook der Physik, Vol. XXII, Berlin: Springer-Verlag, 1956.

7. MeDonald, A. D., "Microwave Breakdown in Gases," New York: John Wiley \& Sons, 1966.

8. Sharbaugh, A. H., and Devins, J. C., "Electrical Breakdown in Solids and Liquids," Electro-Technology, Science and Engineering Series, 97-117, October 1961.

9. D. E. Gray, ed., "American Institute of Physies Handbook," New York: McGraw Hill, 1957.

10. Bunker, E. R., Jr., ed., "Proc. of Workshop on Voltage Breakdown in Electronic Equipment at Low Air Pressures," NASA Technical Memorandum 33-280, December 15, 1966.

11. "The Study of Multipactor Breakdown in Space Electronic Systems," NASA Contractor Report 448, July 1966.

12. Dunbar, W. G., "Corona Onset Voltage of Insulated and Bare Electrodes in Rarefied Air and Other Gases," AFAPL-TR-65-122, June 1966.

13. "Directory/Encyclopedia Issue," Insulation, 13(7):56-59, June/July 1967.

14. Scialdoni, John J., "Dynamic Pressure of a Volume with Various Orfices and Out-Gassing Materials," NASA TMX 63-133, October 1967. 
15. Ludeman, C., "Corona and High Voltage Breakdown of Electrical Conductors in Vacuum," NASA Failure Analysis Report 347-001, January 1965.

16. Burrowbridge, Donald, "A Technique for Simulating the Ionospheric Plasma," NASA TMX 63-226, STAR No. N68-25775, May 1968.

17. Kreugar, F. H., "Discharge Detection in High Voltage Equipment," New York: American Elsevier Publ. Co., 1965.

18. Cazanjian, R. S., and Jacobs, R. M., "Design Review-The Why and the How, "Proc. of the Seventh National Symposium on Reliability and Quality Control in Electronics, New York: IRE, 344-350, 1961.

19. Smith, B. D., and Aptaker, I. M., "A Check List for Design Review," Electronic Design, Part I, 36-39, May 24, 1961; Part 2, 60-63, June 7, 1961. 


\section{BIBLIOGRA PHY}

Bachynski, M. P., "Sources in Plasma," RCA Review, 28:111, 1967.

Brown, S. C., "Basic Data of Plasma Physics," Cambridge, Mass.: MIT Press, 1959.

Bunker, E. R., Jr., ed. , "Proceedings of Workshop on Voltage Breakdown," NASA Technical Manual 33-280, December 1966.

Cazanjian, R. S., and Jacobs, R. M., "Design Review-The Why and the How," Proceedings of 7th National Symposum on Reliability and Quality. Control in Electronics, New York: IRE, 344-350, 1961.

Cobine, J. D., "Gaseous Conductors," New York: Dover, 1958.

Devins, J. C., and Sharbaugh, A. H., "The Fundamental Nature of Electrical Breakclown," Electro-Technology. Science and Engineering Series, 103-122, February 1961.

"Directory/Encyclopedia Issue," Insulation, 13(7), June/July 1967.

Dorrell, R., and Cooke, P., "Tips to Experimenters, "Quality Assurance Branch, GSFC, 1966.

Ellion, E. M. , "A Study of Electrical Discharge in Low Pressure Air," Jet Propulsion I aboratory, Technical Report No. 32-678, April 1, 1965.

Howatson, A. M., "An Introduction to Gas Discharges," New York: Pergamon Press, 1965.

Loeb, I. B. , "Basic Processes of Gaseous Electronics," 2nd edition, Berkeley and Los Angeles: Univ. of Calif. Press, 1960.

Loeb, L. B., "Electrical Coronas," Berkeley and Los Angeles: Univ. of Calif. Press, 1965.

Ludeman, C., "Corona and High Voltage Breakdown of Electrical Conductors in Vacuum," Failure Analysis Report 347-001, January 1965.

McDonald, A. C., "Microwave Breakdown in Gases," New York: John Wiley and Sons, 1966. 
Meek, J. M., and Craggs, J. C., "Electrical Irreakdown of Gases," (oxford: Clarendon Press, 1953.

Mentzoni, M., and Ionohue, J., "Pulsed RF Slot-Antenna," Electronics Letters, 3:104, March 1967.

Olyphant, M., Jr., "Corona and Treeing Breakilown of Insulation," Insulation. February, 35-40; March, 23-27; April, 42-46, 1963.

Reilly, J. P., "Electric Breakdown in Path of Microwave Energy Beam Radiated from Conical Vehicles During Reentry into Earth Atmosphere," Research Rept., Aveo Everett, No. 214, 1965.

Sharbaugh, A. H., and Devins, J. C., "Electrical Breakdown in Solids and Liquids, "Electro-Technology". Science and Engineering Series, 97-117, October 1961.

Smith, B. D., and Aptaker, I. M., "A Check l ist for Design Review, "Electronic Design, Part 1, 36-39, May 24, 1961; Part 2, 60-63, June 7, 1961.

Stair, W. 'T', "High Altitude Flashover and Corona Problems," ElectroTechology, 69:124, May 1962 .

"The Study of Multipactor Brealidown in Space rlectronic Systems," Appendix, Vol. II, Hughes Aircraft Co., Final Report NAS5-3916, April 1965.

"The Study of Multipactor Breakdown in Space Electronic Systems," Hughes Aircraft Co., NASA Contractor Report, July 1966. 